\title{
Organic Lasers - Recent Developments on Materials, Device Geometries, and Fabrication Techniques
}

\author{
Alexander J.C. Kuehne ${ }^{1^{*}}$ and Malte C. Gather ${ }^{2^{*}}$ \\ ${ }^{1}$ DWI - Leibniz Institute for Interactive Materials, RWTH Aachen University, Germany \\ ${ }^{2}$ Organic Semiconductor Centre, SUPA, School of Physics and Astronomy, University of St Andrews, \\ United Kingdom
}

*Email: kuehne@dwi.rwth-aachen.de; mcg6@st-andrews.ac.uk

\begin{abstract}
Organic dyes have been used as gain medium for lasers since the 1960s-long before the advent of today's organic electronic devices. Organic gain materials are highly attractive for lasing due to their chemical tunability and large stimulated emission cross-section. While the traditional dye laser has been largely replaced by solid-state lasers, a number of new and miniaturized organic lasers have emerged that hold great potential for lab-on-chip applications, bio-integration, low-cost sensing and related areas, which benefit from the unique properties of organic gain materials. On the fundamental level, these include high exciton binding energy, low refractive index (compared to inorganic semiconductors) and ease of spectral and chemical tuning. On a technological level, mechanical flexibility and compatibility with simple processing techniques such as printing, roll-to-roll, self-assembly and soft-lithography are most relevant. Here, the authors provide a comprehensive review of the developments in the field over the past decade, discussing recent advances in organic gain materials - today often based on solid-state organic semiconductors - optical feedback structures, and device fabrication. Recent efforts towards continuous wave operation and electrical pumping of solid-state organic lasers are reviewed and new device concepts and emerging applications are summarized.
\end{abstract}




\section{Contents}

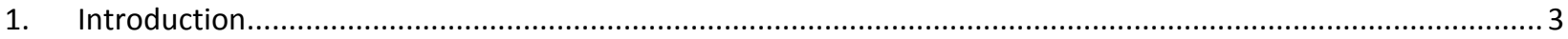

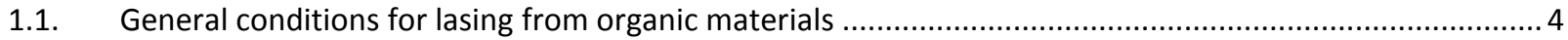

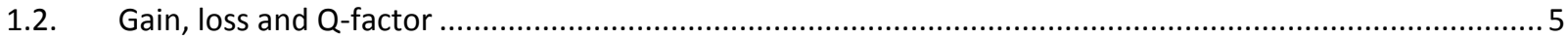

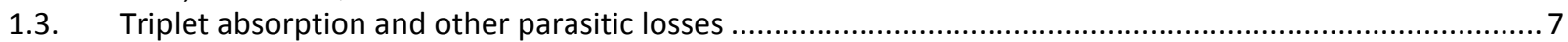

1.4. Amplified spontaneous emission and unambiguous detection of lasing ........................................... 9

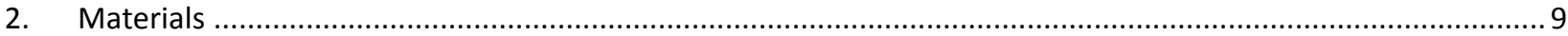

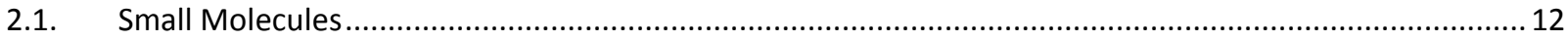

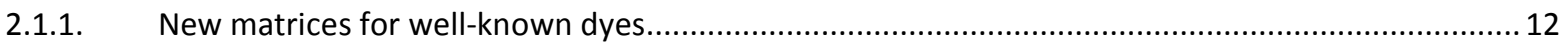

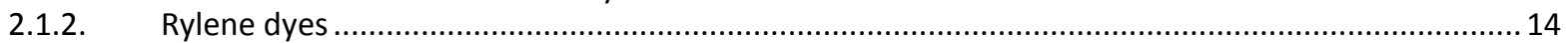

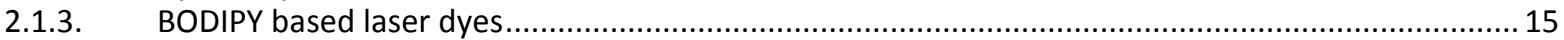

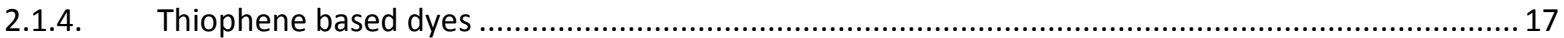

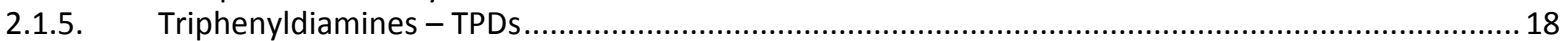

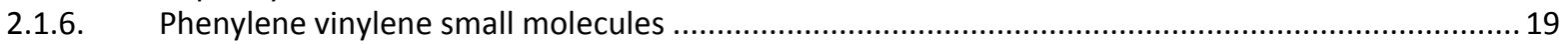

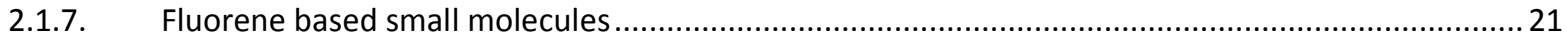

2.1.8. Towards orbital design via precise functionalization with donor and acceptor groups ...................22

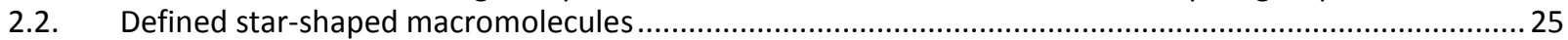

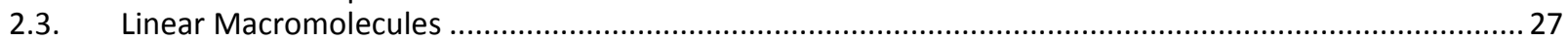

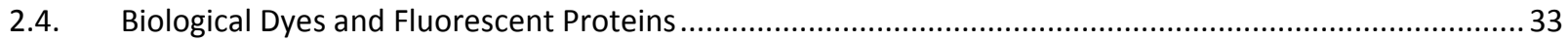

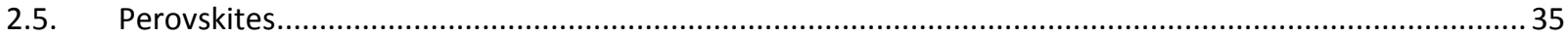

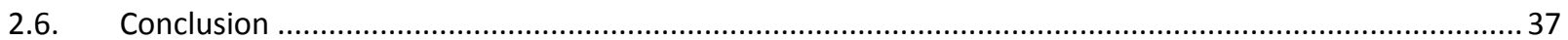

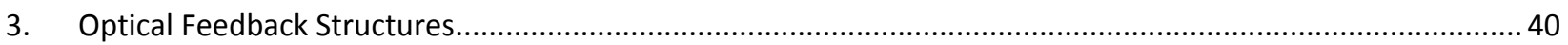

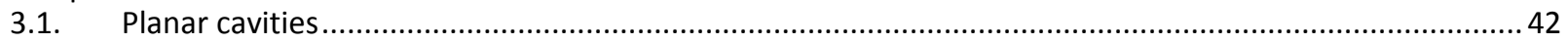

3.2. Distributed feedback and photonic crystal structures ............................................................. 47

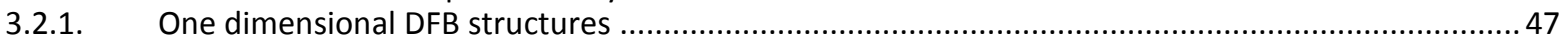

3.2.2. Two and three dimensional photonic crystal structures ........................................................5 53

3.2.3. Development of designs for future electrical operation ...........................................................5

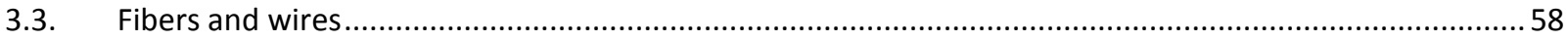

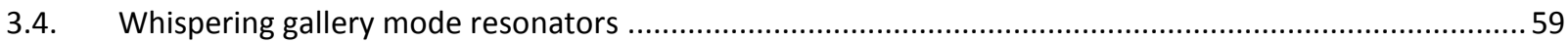

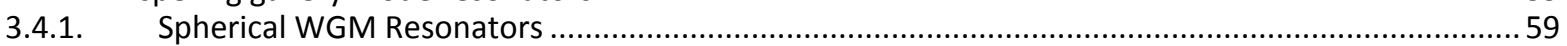

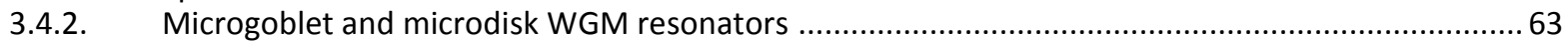

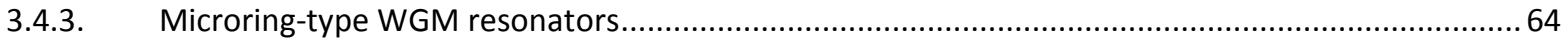

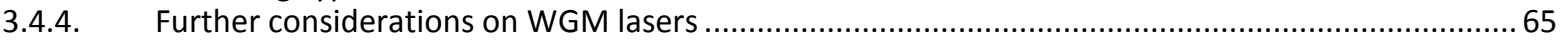

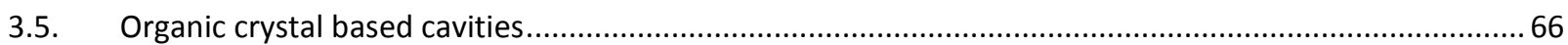

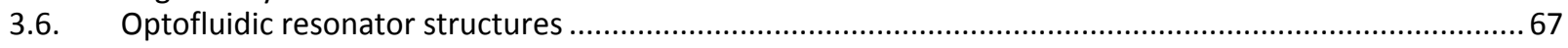

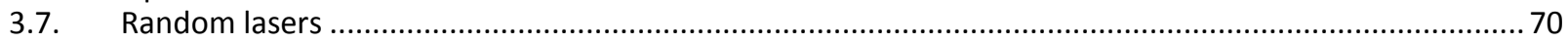

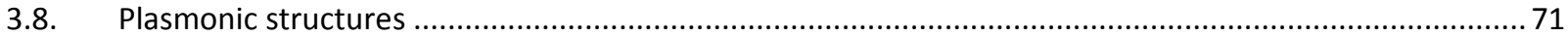

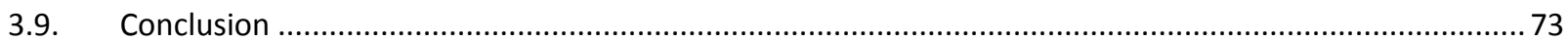

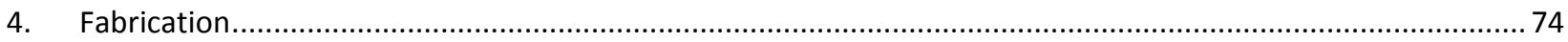

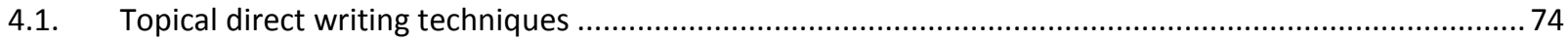

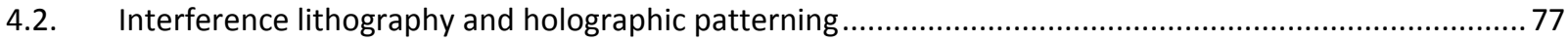

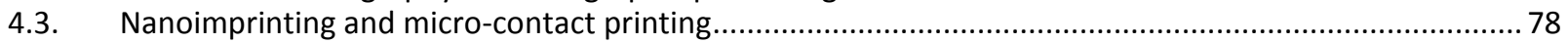

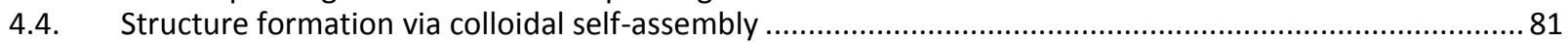

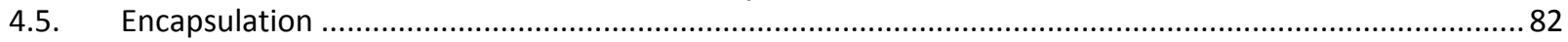

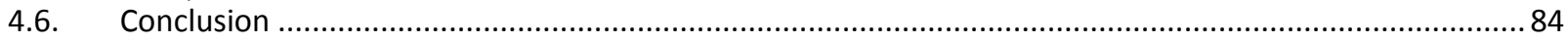

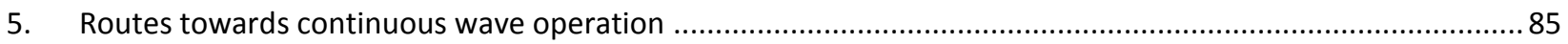

6. Strategies to overcome the challenges associated with electrical pumping of organic lasers ........................ 88

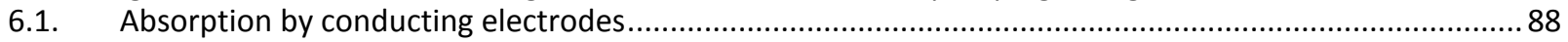

6.2. Increasing charge carrier mobility and reducing excitonic and polaronic losses ..................................91

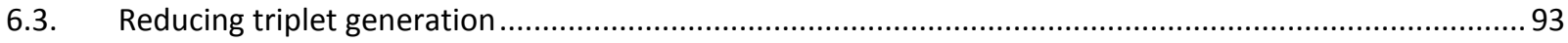

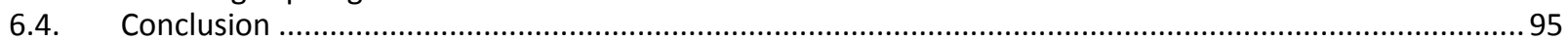

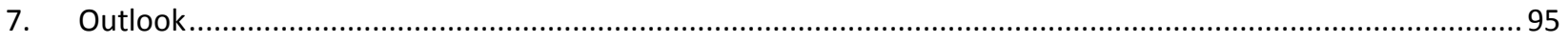

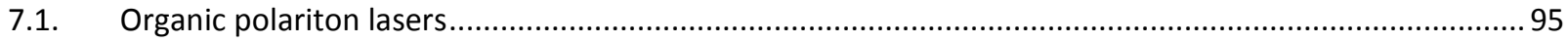

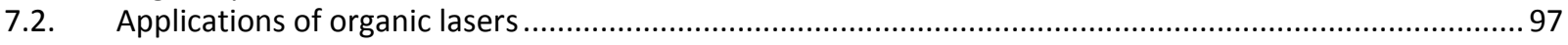

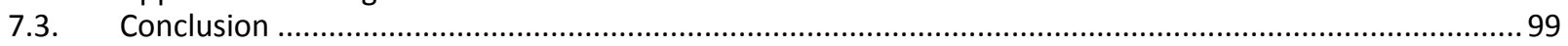

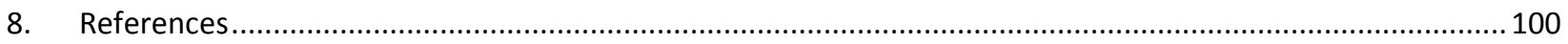




\section{Introduction}

Lasers provide light with unique and extremely useful properties, including high intensity, directionality, monochromatic emission and large coherence length. Due to these properties, lasers have found applications in almost every economic and industrial sector. Lasers are ubiquitous in our everyday life, for example in scanners, printers and sensors. The ability to control the temporal, spectral and spatial characteristics of lasers with extreme precision has transformed the field of spectroscopy and provides record-breaking sensitivity and resolution. Facilitated by the constant development and rapid improvements of lasers, they also continue to enter new fields. For instance, lasers are nowadays also considered for applications in lighting where directed emission and high brightness are required (e.g. car headlamps). In nano-photonics, lasers with deep sub-wavelength dimensions may become the buildingblocks of future optical computing. Until now, lasers for these and other applications are typically based on inorganic emitter materials; in many cases inorganic semiconductors and doped crystals. These materials are generally brittle, non-flexible and their production and processing often requires highly reactive and toxic heavy metal precursors as well as high vacuum equipment. By contrast, organic semiconductor materials are generally easier to process and the resulting devices can be mechanically flexible. In addition, organic emitter materials are often less harmful than their inorganic counterparts and devices based on them have shown excellent biocompatibility. ${ }^{1,2} \mathrm{~A}$ number of classes of organic semiconductors exhibit high optical gain, enabling their use as laser media and optical amplifiers. Due to their facile processability, they are compatible with a large variety of optical resonator structures and in many cases the resonator can be inscribed directly into the organic gain medium, leading to versatile and relatively low cost laser structures.

The last comprehensive review on organic semiconductor based lasers in this journal was published by Samuel and Turnbull in 2007. ${ }^{3}$ A number of review articles and book chapters on different aspects of organic semiconductor lasers have appeared in the meantime ${ }^{2-12}$. Here, we first briefly discuss some 
general considerations relevant to organic lasers before providing an updated comprehensive review on organic gain materials, resonator geometries and innovative fabrication techniques. The sections on each of these aspects begin with a brief introduction of the most important concepts and approaches, followed by a review of the relevant literature, with a strong focus on work published within the past decade. We will also discuss approaches towards continuous wave emission and strategies to overcome problems associated with electrical pumping of organic lasers. Finally, we will give an outlook by discussing emerging devices and applications that are enabled by organic lasers and organic gain materials.

\subsection{General conditions for lasing from organic materials}

Like any laser, organic lasers require three principal components, a gain medium, an optical feedback structure and a pump source. The defining characteristic of an organic laser is usually that the gain medium is based on an organic luminophore, typically a $\pi$-conjugated aromatic hydrocarbon compound. To fully harness the advantages of organic lasers (in terms of mechanical flexibility, biocompatibility, ease of fabrication etc.) many concepts for organic lasers also involve organic materials to define the optical feedback structures. In many cases the gain material itself is in fact shaped such that it provides optical feedback. The pump source for organic lasers is - with very few exceptions - an external, pulsed light source that is directed towards the organic gain material.

In general terms, for a structure to operate as a laser, light trapped within the structure needs to be amplified and the amount of amplification needs to cancel all losses present. An archetypical laser design comprises two mirrors for optical feedback with the organic semiconductor as gain medium filling the space in between (Figure 1a). A fraction of the spontaneous fluorescence emitted by the organic semiconductor hits the mirrors under a suitable angle to be reflected back and forth between the mirrors and to repeatedly pass through the organic material. When interacting with luminophores in the 
organic material that are in their excited state, this light can trigger stimulated emission and thus be amplified. If the amount of amplification equals or exceeds losses (which usually originate from nonperfect reflection at the mirrors and absorption by the material between the mirrors), a continuous avalanche of photons can be sustained. The emitted laser radiation is the light that leaks from the structure, e.g. if one of the mirrors is semi-transparent. Stimulated emission retains the wavelength, phase and polarization of the incident light, meaning that both circulating and emitted light are coherent.

\subsection{Gain, loss and Q-factor}

Mathematically, the condition for sustained lasing is described most conveniently by requiring that the electric field $E_{0}$ of the light propagating back and forth between two mirrors is reproduced after one round-trip, i.e.

$$
E_{0}=E_{0} e^{-i 2 L n k_{0}} \sqrt{R_{1} R_{2} e^{2 L(g-\alpha)}}
$$

Here $L$ is the separation distance between the mirrors, $n$ is the (effective) refractive index, $k_{0}=2 \pi / \lambda_{0}$ is the vacuum wavevector of the light (with $\lambda_{0}$ being the vacuum wavelength), $R_{1}$ and $R_{2}$ are the reflectivity of each mirror, $g$ is the (modal) optical gain and $\alpha$ is the (modal) absorption of the organic material and any other material within the cavity at wavelength $\lambda_{0}$. The imaginary exponential term in the equation above imposes a geometric constraint, i.e. it is only equal to unity if the mirror separation is an integer multiple of the effective wavelength of the circulating light. The square root factor, expresses the material constraint that the amplification, which is described by the modal gain $g$, needs to be equal to or exceed the losses present in the structure, i.e.

$$
g \geq \alpha-\frac{\ln R_{1} R_{2}}{2 L}
$$


To generalize this expression for other optical feedback structures (other than the pair of mirrors considered so far), one can introduce the $Q$-factor, which describes the ability of any feedback structure to retain light. For the simple pair of mirrors, the inverse of the $Q$-factor can be expressed by $1 / Q=$ $\left(\alpha-\frac{\ln R_{1} R_{2}}{2 L}\right) \frac{\lambda_{0}}{2 \pi n}$. The general form of the above amplification constraint is therefore

$$
g \geq \frac{2 \pi n}{\lambda_{0} Q}
$$

The modal gain available in a material increases with the density of excited-state luminophores, which in turn depends on the level of strength of pumping. This gives rise to the characteristic threshold behavior of lasers, where lasing action only starts once the pumping is sufficient to provide a large enough density of excited state luminophores for the gain to compensate loss. At large, one will try to minimize the modal gain required to achieve lasing in order to minimize the pump energy required. From the above it is clear that this minimization requires simultaneous optimization of both the optical feedback structure (the $\frac{\ln R_{1} R_{2}}{2 L}$ term) and the absorption $\alpha$ of the organic material at the lasing wavelength. Strategies towards efficient optical feedback structures are discussed in section 3. To understand the challenges of ensuring low material absorption, one has to look at the photophysics of organic semiconductors. In particular during laser action, this can be rather complex, often involving a number of different excited states of the material with individual absorption profiles. To first approximation, however, many organic semiconductors are efficient quasi-four level laser systems. This is conveniently seen by considering a generalized Jablonski diagram of an organic luminophore (Figure 1b). By optical pumping, the luminophore is excited from the electronic ground state $S_{0}$ (Level 1 ) to the first electronic excited state $S_{1}$ (Level 2), a process during which vibrational modes are usually also excited. Most materials relax back to the lowest vibrational level of $\mathrm{S}_{1}$ within less than a pico-second (Level 3). Stimulated and spontaneous emissions occur from Level 3 to one of the higher vibrational level of the electronic ground state manifold (Level 4). Vibrational relaxation back to Level 1 is again very fast, which prevents built up of any 
significant population of luminophores in Level 4. In addition, prompt relaxation from Level 2 to Level 3 allows easy population of the Level 3 state. This means that population inversion, i.e. more luminophores in Level 2 than in Level 3, can be readily achieved in these materials.

(a)

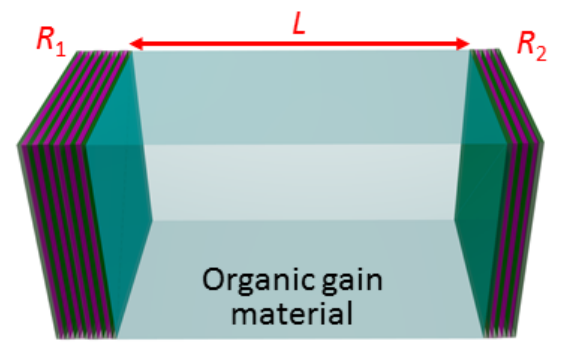

Mirror 1

Mirror 2

(b)

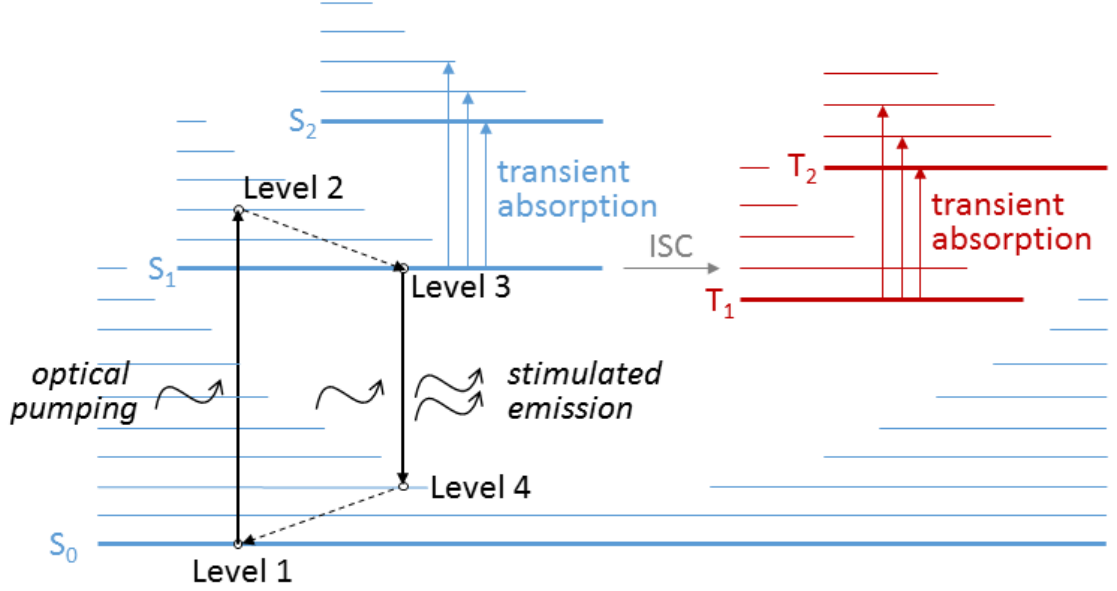

Figure 1: (a) Archetypical structure of an organic laser, with two mirrors with reflectivity $R_{1}$ and $R_{2}$, separated by distance $L$, and organic gain medium in between. (b) Generalized Jablonski diagram of an organic luminophore illustrating how the luminophore can act as a quasi four level laser system. Fast vibronic relaxation of most organic luminophores after electronic excitations allows efficient population of the state at the bottom of the $S_{1}$ manifold (Level 3 ) and prevents accumulation of luminophores in higher vibronic states of the $\mathrm{S}_{0}$ manifold (Level 4). Losses originated from intersystem crossing (ISC) between the first excited singlet state $\left(\mathrm{S}_{1}\right)$ and the $\mathrm{T}_{1}$ triplet manifold and from subsequent transient absorption to higher triplet states.

\subsection{Triplet absorption and other parasitic losses}

Together with other factors, the vibrational relaxation during the excitation/emission cycle leads to a considerable Stokes shift for many organic materials, where the emission and absorption bands become well-separated and there is only weak overlap between the absorption and the emission spectra of the material. This translates into low absorption $\alpha$ at the lasing wavelength and facilitates lasing even if the 
modal gain $g$ is relatively low. However, there are a number of parasitic effects that can introduce absorption at the lasing wavelength and thus reduce the laser output or prevent lasing altogether. The most prevalent parasitic effect is absorption from triplet states. When in their excited $\mathrm{S}_{1}$ state, organic luminophores have a small probability to undergo intersystem crossing into the first triplet state $T_{1}$ instead of relaxing back to the $S_{0}$ ground state. The $T_{1}$ state is only very weakly emissive and often longlived because the transition to the $\mathrm{S}_{0}$ ground state is spin-forbidden (average time to relaxation can be milliseconds or even seconds). However, the luminophore can undergo an optical transition to a higher excited triplet state $T_{n}$ and the excited state absorption spectrum for this process often overlaps with the emission spectrum of the luminophore. Triplet absorption is unproblematic immediately after pumping of the laser begins; however, over time more and more luminophores may accumulate in the triplet state and their absorption can inhibit lasing after as little as a few nanoseconds of operation, thus preventing continuous wave $(\mathrm{CW})$ operation for most organic lasers. Traditionally the issue of triplet accumulation has been circumvented by quickly replenishing the organic material in the laser, e.g. with a dye jet solution. Alternatively, nanosecond excitation pulses can be used to pump the laser when the repetition rate is adjusted such that there is sufficient time for triplet states to decay in between pulses, i.e. the duty cycle is reduced. More recently, other strategies for preventing triplet accumulation have been suggested and these will be discussed in sections 5 and 6.3 .

In addition to triplet absorption, there are a number of other absorption processes potentially detrimental to lasing. The excited state absorption of the $S_{1}$ state may also overlap with the emission spectrum. Furthermore, work towards developing an electrically pumped organic laser has proven to be very difficult due to absorption of charged organic molecules and electric-field induced absorption effects. These aspects will be discussed in detail in section 6 . 


\subsection{Amplified spontaneous emission and unambiguous detection of lasing}

Amplified spontaneous emission (ASE) is a process related to lasing. In the absence of an optical feedback structure, photons that are emitted by a luminophore through spontaneous fluorescence can still be amplified by stimulated emission if they interacting with other excited luminophores in the gain material. Through further stimulated emission, this can trigger an avalanche of photons known as ASE. ASE has characteristics somewhat similar to laser emission. In particular, the spectral linewidth of the emission is generally narrowed compared to the spontaneous fluorescence spectrum and there is in many cases some form of threshold behavior, i.e. the intensity of emission increases more rapidly beyond a certain threshold pump fluence. Measurements of the ASE behavior of a material can be used to quantify the gain available in a certain material and allow enable the characterization of gain materials independent of an optical feedback structure.

ASE and several other effects can also be falsely interpreted as laser activity and thus complicate the unambiguous identification of lasing in organic lasers. Particular care has to be taken when evaluating claims of lasing under electrical rather than optical pumping. ${ }^{13}$ Generally, the linewidth narrowing and the threshold behavior are less pronounced for ASE than for lasing. ASE linewidth are typically in the range of a few $\mathrm{nm}$ whereas for single mode laser operation the total linewidth will be well below $1 \mathrm{~nm}$. Other processes that may falsely interpreted as lasing include edge-emission of waveguided modes, which often show strong line-narrowing compared to the spontaneous fluorescence spectrum of the

organic material but are not expected to show threshold behavior. ${ }^{14}$ Superfluorescence or superluminescence and thermo-optic effects may also be mistaken for lasing.

\section{Materials}

Materials for organic laser gain media can be obtained from molecules with a wide spectrum of molecular weights. This includes small molecules which in the context of organic semiconductors are 
molecules with molecular weights of less than around 1,000 Da, star-shaped and dendritic emitter materials comprising a defined number of covalently linked luminophores, and linear polymers, which typically have molecular weights of up to a few hundred kDa and comprise up to several hundred repeat units. In all of these cases, the molecules will contain one or several luminophores based on conjugated $\pi$-electron systems. The luminophore units are typically functionalized with non-conjugated segments, which confer improved solubility or have steric function acting on the intermolecular packing of the materials.

For small molecule materials in particular, close packing often leads to self-quenching of luminescence, an effect well known from classical dyes and pigments. In organic pigments, the dyes molecules are closely packed so that self-quenching occurs and absorption dictates the resulting color, which is complementary to the absorption profile. Functionalization with sterically demanding aliphatic and branched chains can reduce the effect of quenching to some extent, inducing fluorescence. Alternatively, low molecular weight gain materials can be dissolved in a dielectric matrix, which is usually a solvent or a transparent polymer. Small molecules without such a matrix or sterically demanding periphery tend to crystallize, leading to undesired quenching as mentioned above. However, single crystalline materials remain a topic of active research. The confinement of the crystal lattice can inhibit ingress of oxidants and fixes the molecular arrangement into a densely packed structure. This helps to improve general stability of the material and in particular can reduce issues with photo oxidation, which may otherwise limit the useful lifetime of organic lasers. In addition, crystalline materials can offer a high density of luminophores, which results in large optical gain if self-quenching can be avoided. Finally, the resulting symmetrical crystal geometries can be employed as self-assembled laser resonators by exploiting internal total reflection at the crystal boundaries. Hexagonal disk shapes, for example, can be used as whispering gallery mode resonators, while single crystals with parallel facets act as Fabry-Pérot or microcavity resonators. These geometries will be discussed in section 3. 
While small molecules can often be processed by thermal evaporation under high vacuum and also from solution if suitable solubilizing side groups are present, the molecular weight and evaporation temperature of dendrimers and oligomers is generally too high for vacuum processing. However, they are well suited for solution processing and usually form amorphous smooth films with high optical uniformity and transparency. Like small molecules, they can still be purified via column chromatography so that materials with high purity, good molecular definition (i.e. minimal defects, side-products and catalyst residues) can be obtained. For dendrimers in particular, self-quenching can largely be suppressed, thus avoiding the need for a dielectric matrix. However, the high definition comes at the cost of more complicated synthetic procedures; as dendrimers are built up sequentially - generation by generation - multiple coupling reactions and purification steps are required to achieve the desired final product.

By contrast, high molecular weight polymeric gain materials can often be produced in a single polymerization step. However, the high molecular weight complicates purification. Polymers are typically purified using precipitation and extraction methods, impeding the complete removal of polymer chains with defects and residual catalyst. In addition, it has proven difficult to reduce polydispersity, and in most materials there is a significant distribution of polymer chain lengths. The laser performance of polymeric gain materials hence strongly depends on the purification procedure and can vary from batch to batch. Nevertheless, polymers have proven highly efficient for circumventing the issue of crystallization and aggregation. The viscous nature of most polymer solutions enables preparation of smooth thin films using simple solution processing methods.

In the following we present a summary of the latest materials and approaches to organic gain materials with particularly high performance. To compare the different materials, we assess the thresholds for detectable ASE and lasing. We have opted to compare the required energy density (i.e. threshold fluence in energy per area), as this data can be extracted from most reports. However, we acknowledge that 
there are other important parameters to characterize gain materials such as photoluminescence quantum yield (PLQY), gain and the power density at threshold and we will mention values for these where relevant.

\subsection{Small Molecules}

Small molecule laser dyes have a long history and are in fact nearly as old as the laser itself. The classic dye laser is based on a dye solution that is circulated through an optical cavity and pumped with an external source (often a $\mathrm{N}_{2}$-laser). Circulation of the dye is implemented to prevent accumulation of dye molecules in their triplet state and to enable CW operation. By selecting appropriate dyes, laser light with wavelengths across the entire visible and parts of the near infrared spectrum can be generated. However, the advent of compact supercontinuum sources and optical parametric oscillators (OPOs) and amplifiers (OPAs) has made dye lasers somewhat redundant; especially since handling of the required dye solutions can be troublesome and has frequently raised health and safety concerns.

Today, however, incorporation of dyes into solid matrices or as molecular glasses and thin films again generates substantial interest as this enables relatively simple fabrication of laser sources with micron scale dimensions. However, working in solid-state implies that problems associated with triplet accumulation and photostability have to be addressed more carefully, e.g. by improving resonator quality and the optical gain available from the materials. A variety of different approaches have been pursued, ranging from new resonator geometries (see section 3 ) to new synthetic concepts (described below).

\subsubsection{New matrices for well-known dyes}

In order to teach 'old' dyes new tricks for laser applications, one can either change their host matrix or carefully modify the dye structure to add additional functionality. Cyanines represent a class of dyes with high fluorescence yield and good photostability. Near infra-red emitting lasers have been achieved by 
incorporating 1 wt\% of a hemi-cyanine $(\mathrm{HC})$ into a fluorinated polyimide matrix in a planar waveguide structure with polished end caps. The emission wavelength was at $970 \mathrm{~nm}$, which is among the longest wavelength reported for dye-doped polymer lasers today. The lasing threshold, however, was relatively high $\left(220 \mu \mathrm{J} / \mathrm{cm}^{2}\right)^{15}$

Rhodamines are another prominent class of dye molecules. By incorporating $1 \mathrm{wt} \%$ of sulforhodamine (SRh) into a DNA matrix, a solution processable laser formulation was obtained. The solution was spin coated onto a distributed feedback (DFB) grating defined in glass (see section 3.2 for details on the DFB geometry). The DNA/SRh mixture has a refractive index of approximately 1.5 , thus forming a weak waveguide on the slightly lower refractive index glass substrate. The lasing threshold was ca. $30 \mu \mathrm{J} / \mathrm{cm}^{2}{ }^{2}{ }^{16}$

Polymethylmethacrylate (PMMA) has been widely used as host matrix for laser dyes. More recently, by adding a photo-switchable unit to a $1 \mathrm{wt} \%$ rhodamine doped PMMA system, modulation of the threshold and the emission wavelength of the laser became feasible. In one example, $20 \mathrm{wt} \%$ of spiropyrane was added. Upon irradiation with UV light, the spiropyranes were shown to transform into a merocyanine structure. This change in molecular structure also changed the electronic and spectral features of the molecule. In particular, the absorption was bathochromically shifted by more than $200 \mathrm{~nm}$ for the merocyanine isomer and partially overlapped with the rhodamine gain medium. The merocyanine structure converted back into the spiropyrane structure upon irradiation with visible light or also in the dark through a thermally driven process. It was shown that a material formulation with $1 \mathrm{w} \%$ rhodamine, 20 wt\% spiropyrane and 79 wt\% PMMA can be solution processed. When used with a DFB laser resonator produced by nanoimprint lithography (grating period of $\Lambda=510 \mathrm{~nm}$ ), the unexposed system had a threshold of ca. $50 \mu \mathrm{J} / \mathrm{cm}^{2}$ and the emitted laser light had a wavelength of $747 \mathrm{~nm}$. When exposing the system to UV light to switch the spiropyrane into the merocyanine isomer, the laser wavelength shifted to $760.5 \mathrm{~nm}$, accompanied by an increase in threshold. The molecule could be repeatedly switched between the merocyanine and spiropyrane conformation and the laser emission switched 
accordingly. However, each cycle increased the laser threshold, indicating fatigue of the switchable unit or degradation to other parts of the structure. ${ }^{17}$ Nonetheless, this study represents an interesting 'organic' example of $Q$-switching, i.e. of a controlled alteration of the quality of the laser resonator. ( $Q$ switching is widely used in inorganic lasers to generate pulsed emission with ns pulse durations.)

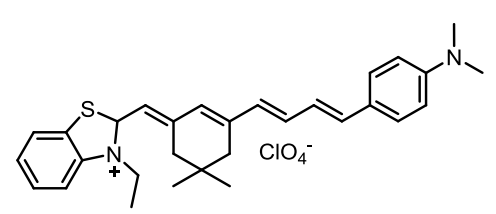

HC

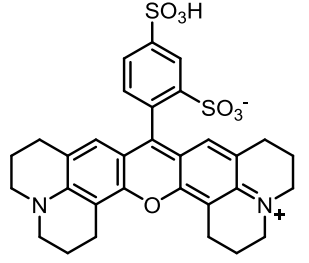

SRh
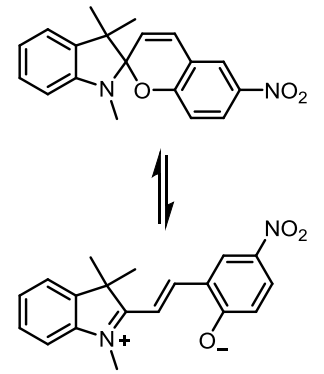

SPy

\subsubsection{Rylene dyes}

Rylene dyes are a class of materials derived from pigments. As discussed above, pigments are particles of crystallized dye molecules, which acquire their excellent stability against photo-oxidation from their crystalline structure. However, crystallinity frequently causes self-quenching and thus inhibits fluorescence. By attaching alkyl or other bulky side groups, the crystallization of rylene molecules was inhibited, resulting in fluorescent dyes with high PLQYs. ${ }^{18}$ By tuning the amount of fused rings in the core of the rylene, the emission wavelength was controlled. The most prominent and smallest representative of the rylene family is perylene. Several studies describe the functionalization of perylenes with various alkyl and phenalkyl solubilizing units (e.g. IND-PBI, IPP-PBI, EH-TBP-PBI). Recent studies have looked at $\mathrm{N}, \mathrm{N}$-bis isononadecyl perylene bisimide (IND-PBI) incorporated in a polystyrene (PS) matrix at concentrations between 0.5 and $5 \mathrm{wt} \%$. Thresholds for ASE as low as $150 \mu \mathrm{J} / \mathrm{cm}^{2}$ were determined for optimum concentrations (between 0.75 and $1 \mathrm{wt} \%$ of perylene in matrix). ${ }^{18}$ The material mix can be spin coated onto DFB resonators and lasing thresholds of $20-30 \mu \mathrm{J} / \mathrm{cm}^{2}$ were obtained. ${ }^{19}$ The same authors 
also investigated the optimal film and waveguide thickness for these lasers as well as the ideal depth of the DFB structure. ${ }^{20,21}$

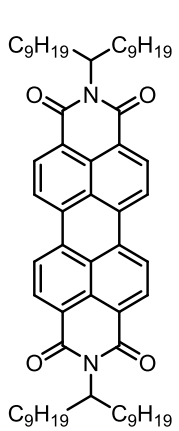

IND-PB

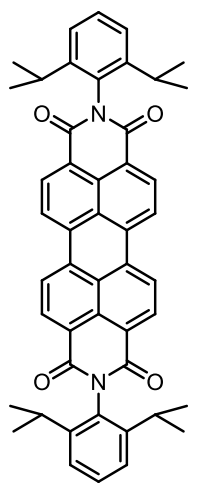

|PP-PBI

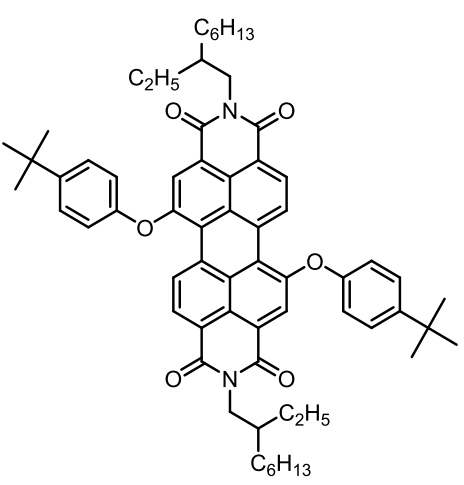

EH-TBP-PBI

In a different study, single crystals of 4-fold core-arylated perylene bisimides were obtained via Rucatalyzed arylation. This compound crystallized into hexagonal micro-disks with diameters of several microns, which sustained whispering gallery mode resonances (see section 3 for details on whispering gallery modes). These crystals exhibit fast fluorescence decay rates, fluorescence yields of around $15 \%$ and laser thresholds of $10.8 \mu \mathrm{J} / \mathrm{cm}^{2} .22$

\subsubsection{BODIPY based laser dyes}

BODIPYs are a rather young class of dye materials for laser applications and so we will also briefly summarize their recent application in conventional solution-based dye-lasers. BODIPYs are composed of a pyrromethene unit, which coordinates to borondifluoride. BODIPYs are of interest due to their high PLQY, often approaching 100\%. BODIPYs offer a variety of sites for functionalization and the position has an impact on the fluorescence and the stimulated emission characteristics. The 3 and 5 sites can be easily functionalized with methyl groups, which entail a bathochromic shift. Functionalization of the 8 position with hetero atoms resulted in meso-functionalized BODIPYs leading to a hypsochromic shift in emission wavelength. In this way, a variety of BODIPY dyes was produced with laser emission wavelengths in the blue to green spectrum. ${ }^{23}$ Lasing from BODIPY dyes was investigated in various polar 
solvents using a Fabry-Pérot geometry (see section 3.1 for description of Fabry-Pérot resonators). When functionalized with alkyl side groups, the compound became soluble in non-polar organic media and matrices (e.g. ${ }^{\mathrm{n}} \mathrm{Bu}-{ }^{\mathrm{t}}{ }^{\mathrm{Bu}}$-BODIPY). BODIPYs can also act as lasing media when dissolved in commercial liquid crystalline media. At between 0.5 and $2 \mathrm{wt} \%$ loading in a liquid crystalline matrix, BODIPYs exhibited lasing between $570-590 \mathrm{~nm}$ with thresholds $<5 \mu \mathrm{J} /$ pulse and it was shown that BODIPYs perform better in terms of threshold and dye loading than a standard laser dye such as DCM. ${ }^{24}$ Introduction of a propargylamino-unit at the 8 position of BODIPY (PrgA-BODIPY) led to a rotameric molecule with emission in the blue $(483 \mathrm{~nm})$ when pumped with $355 \mathrm{~nm}$ excitation light. This PrgABODIPY exhibited stable laser emission when dissolved in ethyl acetate and placed in a grazing incidence tunable grating resonator. The dye was relatively stable, with bleaching experiments showing that the emission intensity only dropped by $21 \%$ after 18,000 excitation pulses; however, in this study the excitation power was kept below the lasing threshold. Nevertheless, the measurements still show that PrgA-BODIPY is more stable than established laser dyes emitting in a similar spectral range, such as Coumarin $480 .{ }^{25}$ When functionalizing both, the pyrromethene 3,5-positions as well as the 8-position, the electronic properties of BODIPY can be finely tuned. When flanking the BODIPY motif with two EDOT units and meso-substituting with a nitrophenyl moiety, precise tuning of the HOMO and LUMO distribution became possible (meso-NP-EDOT-BODIPY). The HOMO was concentrated over the EDOT units, while the LUMO resided in the nitrophenyl group. These geometries might in the future become interesting for molecules with precise alignment of the frontier molecular orbitals, as will be discussed in section 2.1.8. ${ }^{26}$ 


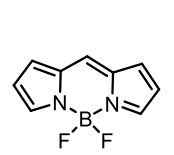

BODIPY meso-BODIPY

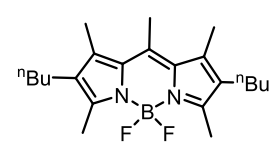

n'Bu-BODIPY

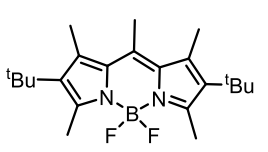

tBu-BODIPY

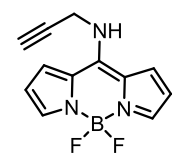

PrgA-BODIPY

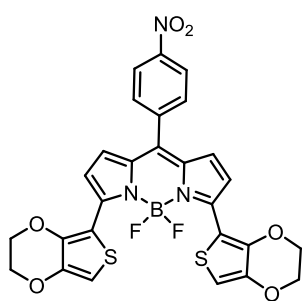

meso-NP-EDOT-BODIPY

\subsubsection{Thiophene based dyes}

The fluorescence of crystalline small molecule pigments can usually be enhanced by separating the $\pi$ conjugated units using bulky side groups like alkyl-chains. This functionalization of the $\pi$-conjugated core inhibits intermolecular quenching and increases solubility. Generally, quenching in molecular crystals is most pronounced for crystal geometries, for which the dipole-dipole interactions are aligned, i.e. when the $\pi$-systems are stacked. There are crystal symmetries, however, in which neighboring molecules are arranged such that there is no $\pi$-stacking, for example if molecules stack in a herringbone or end-to-end fashion. For instance, it was shown that alternating thiophene ( $T$ ) - phenylene (P) pentamers (PTPTP; 1,4bis(5-penylthiophen-2-yl)benzene) crystallize in this way. PTPTPs form thin hexagonal crystals and the crystal faces were used as cavity-mirrors. When excited with short pulses of $420 \mathrm{~nm}$ light, the crystal emitted green laser emission at around $550 \mathrm{~nm}$ with a threshold of $880-1100 \mu \mathrm{J} / \mathrm{cm}^{2} .{ }^{27}$ In another study a thiophene-phenylene hexamer (PPTTPP; 5,5'-bis(4'-methoxybiphenyl-4-yl)-2,2'-bithiophene) was crystallized into a single crystalline lozenge. When excited at $365 \mathrm{~nm}$ wavelength, these crystals produced green emission around $525 \mathrm{~nm}$. The lasing threshold was $208 \mu \mathrm{J} / \mathrm{cm}^{2}$ and thus much lower than for the PTPTP pentamer. In addition, it was shown that these single crystal lasers can be operated under ambient conditions without significant photo-oxidation, even at high excitation densities. ${ }^{28}$

Molecular glasses based on dithienylbenzothiadiazole (DTBT) were found to exhibit even lower lasing thresholds. Various bulky aromatic and alkyl groups were fused with the DTBT core unit to separate the emitter units, thus preventing quenching and allowing for solution processing, e.g. by spin-coating or ink- 
jet printing. The substitution with aromatic, alkyl or alkoxy units also influenced the electronic structure of the molecules, thus shifting the absorption and fluorescence maxima. Glassy films on a DFB grating with a period $\Lambda=410 \mathrm{~nm}$ were produced via spin-coating and lasing was observed at $650 \mathrm{~nm}$ for excitation fluences above $118 \mu \mathrm{J} / \mathrm{cm}^{2}\left(\lambda_{\text {ex }}=410 \mathrm{~nm}\right) .^{29}$

Fused thiophenes, for example pentathienoacenes (PTA), were incorporated into common host matrices such as PS, where they exhibited ASE $\left(10 \mathrm{wt} \%, E_{\mathrm{th}}=100 \mu \mathrm{J} / \mathrm{cm}^{2}\right)$. Dioxides of pentathioneacenes were also incorporated into non-oxidized pentathienoacene matrices, which then facilitated exploiting energy transfer phenomena from host to dopant and thus yielded lower ASE thresholds than in PS hosts. In this way, thresholds of $370 \mu \mathrm{J} / \mathrm{cm}^{2}$ were obtained at emission wavelengths between 500 and $550 \mathrm{~nm}$ for the pentacienoacene-4,4-dioxide and 550 to $600 \mathrm{~nm}$ for the pentacienoacene-9,9-dioxide, respectively. ${ }^{30}$
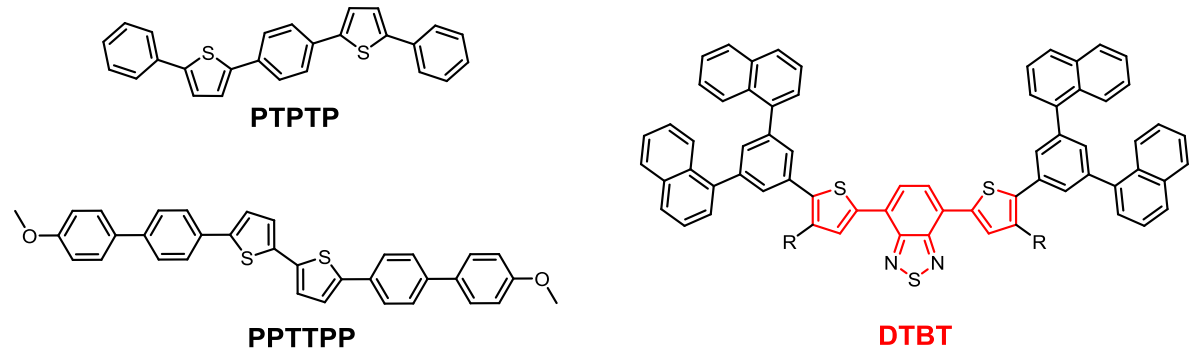

DTBT
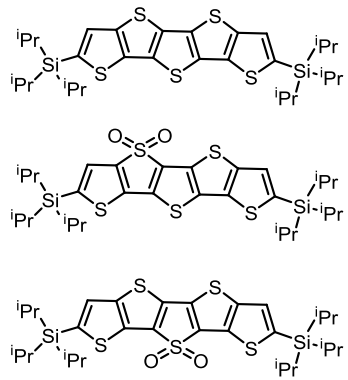

PTA

\subsubsection{Triphenyldiamines - TPDs}

Triphenyldiamines (TPDs) are well known hole transport materials and often form one of the layers in organic light-emitting diode (OLED) and organic solar cell stacks. However, individually, TPDs are light emitting materials with emission in the deep blue spectrum. $N, N^{\prime}$-bis(3-methylphenyl)- $N, N^{\prime}$ diphenylbenzidine is a well-studied TPD, which showed ASE between 415 and $427 \mathrm{~nm}$ when mixed into a PS matrix at $30 \mathrm{wt} \%$. When this mixture was spin-coated from toluene onto a DFB-grating with $\Lambda=$ $270 \mathrm{~nm}$ and excited at $355 \mathrm{~nm}$, the TPD doped film emitted $418 \mathrm{~nm}$ laser light above a threshold of 
$10 \mu \mathrm{J} / \mathrm{cm}^{2} .{ }^{31}$ A net optical gain of $16 \mathrm{~cm}^{-1}$ was determined for this material. ${ }^{32}$ However, the literature on TPDs for lasing applications remains somewhat limited so far.
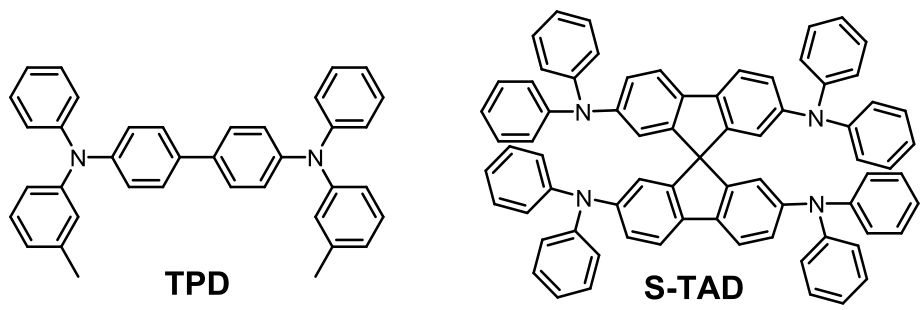

\subsubsection{Phenylene vinylene small molecules}

The most widely known organic conductors based on the phenylene vinylene molecular motif are probably the phenylene vinylene polymers (PPVs), with the PPV commercialized under the trade name Super Yellow being the most prominent representative. However, there are also potent small molecules based on this structure. Trans-1,4-distyrylbenzene (DSB), essentially a short fragment of a PPV polymer, for example has become an important small molecule emitter. Single crystals of DSB can be grown from the gas phase and these showed broad emission in the blue part of the spectrum. The gas phase crystal growth process allowed incorporation of tetracene or pentacene into the crystals at up to $10 \%$, which shifted the emission into the green and the red spectrum, respectively. Pure DSB crystals exhibited a PLQY of $65 \%$. The tetracene doped crystals showed PLQYs of up to $74 \%$, while pentacene reduced the PLQY to 28\%. In line with this, ASE thresholds were lowest for the tetracene doped crystals $\left(0.6 \mathrm{~mJ} / \mathrm{cm}^{2}\right) .{ }^{33}$

DSB can also be functionalized to tune its properties. In single crystals of (1,4-phenylene)bis(3phenylacrylonitrile) (cyano-DSB), molecules were reported to arrange into J-aggregates, resulting in exciton coherence lengths of 2-3 nm. When the crystal was excited at $\lambda_{\mathrm{ex}}=355 \mathrm{~nm}$, multi-peak lasing was observed between 590 and $600 \mathrm{~nm}$ (threshold, $4.8 \mathrm{~mJ} / \mathrm{cm}^{2}$ ). The authors attributed this to random 
lasing, even though the exact nature of the optical feedback remained somewhat unclear and may be the result of defects in the crystal or the crystal facets. Interestingly, when the crystal was excited close to its electronic band-edge at $\lambda_{\mathrm{ex}}=532 \mathrm{~nm}$, single mode lasing at a wavelength of $\lambda_{\text {las }}=580.8 \mathrm{~nm}$ and a reduction in threshold to $2.1 \mathrm{~mJ} / \mathrm{cm}^{2}$ were observed. The authors attributed the single mode lasing to stimulated resonance Raman scattering. ${ }^{34,35}$

To analyze the influence of crystal packing on the laser characteristics of DSB, para- and orthomethylated DSBs were compared. While the $p$-DSB is molecularly flat, the two terminal benzene rings of o-DSB were found to be twisted out of plane by about $30^{\circ}$. Single crystals of both species can be prepared using a vapor transport method. The $p$-DSB crystals are orthorhombic $(P b c a ; Z=4)$, while the $o$ DSB crystals are monolithic $(P \overline{1} ; Z=2) . p$-DSB and $o$-DSB crystals with thickness below $1 \mu \mathrm{m}$ both showed ASE at around $460 \mathrm{~nm}$ when pumped with $500 \mathrm{ps}$ pulses at $\lambda_{\mathrm{ex}}=337 \mathrm{~nm}$. 0 -DSB exhibited a very low threshold of only $14 \mu \mathrm{J} / \mathrm{cm}^{2}$, while with $24 \mu \mathrm{J} / \mathrm{cm}^{2}$ the threshold for $p$-DSB was somewhat higher. ${ }^{36}$

By terminal functionalization of cyano-DSB with alkoxy or fluorinated arylalkoxy, a liquid crystalline material was obtained. When incorporated into a commercial liquid crystalline matrix and co-doped with 4 wt\% of a high twisting-power chiral dopant, the cyano-DSB adopted a chiral nematic phase. The authors postulated that the resulting liquid crystal shows an optical band-gap with a band edge overlapping with the fluorescence spectrum of cyano-DSB. By exciting the sample at $\lambda_{\mathrm{ex}}=355 \mathrm{~nm}$, a sharp laser peak at $470 \mathrm{~nm}$ was obtained for pump fluences above $20 \mathrm{~mJ} / \mathrm{cm}^{2} .{ }^{37}$

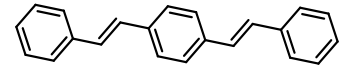

DSB

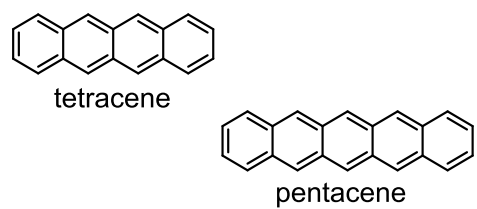

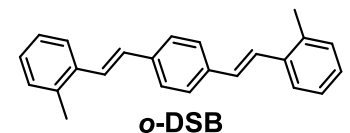

o-DSB

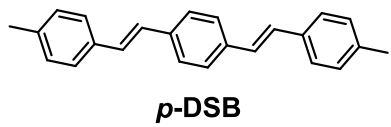

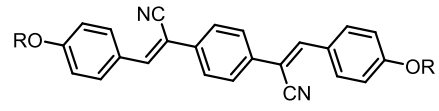

cyano-DSB

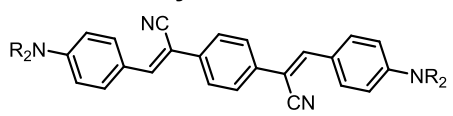




\subsubsection{Fluorene based small molecules}

Fluorene is a relatively inexpensive molecule, which can be easily functionalized with solubilizing units and halide functionalities that enable cross-coupling reactions and thus facilitate generation of larger macromolecules. Fluorenes are usually solids, even when functionalized with alkyl chains in the 9,9'position. Double functionalization of fluorene with longer and branched alkyl groups leads to viscous liquids after synthesis that crystallize slowly over time. However, when functionalized with short siloxane groups, fluorene and short fluorene homologues can be obtained as liquids. Such liquid state fluorene dimers and trimers exhibited ASE thresholds of $1.4 \mu \mathrm{J} / \mathrm{cm}^{2}$ and $22 \mu \mathrm{J} / \mathrm{cm}^{2}$, respectively. ${ }^{38}$ The molecules were non-volatile and exhibited charge carrier mobilities similar to other fluorene based glassy thin films. Alternatively, liquid ethylhexyl functionalized carbazole represents a good host and solvent for fluorene based emitters. When dissolving a heptafluorene in liquid carbazole, the resulting non-volatile emitter blend can be spread over DFB gratings to form relatively efficient lasers; for a $10 \mathrm{wt} \%$ content of heptafluorene lasing thresholds as low as $2.2 \mu \mathrm{J} / \mathrm{cm}^{2}$ were reported. ${ }^{39}$

More complex fluorene architectures that are on the verge to star-shaped and dendritic structures were also investigated. In particular the difluorene cored molecules BP, ES and BPCz were studied. ${ }^{40}$ All three show fluorescence spectra that are dominated by the fluorene units, with maxima between 400 and $440 \mathrm{~nm}$. Their PLQYs increase and ASE thresholds decrease in the order BPCz $\left(\phi=43 \%, E_{\mathrm{th}}=50 \mu \mathrm{J} / \mathrm{cm}^{2}\right)$, $\mathrm{ES}\left(\phi=52 \%, E_{\mathrm{th}}=25 \mu \mathrm{J} / \mathrm{cm}^{2}\right)$ and $\mathrm{BP}\left(\phi=92 \%, E_{\mathrm{th}}=16 \mu \mathrm{J} / \mathrm{cm}^{2}\right)$. These thresholds are comparable to the threshold of the fluorene based polymer poly(dioctylfluorene) (PFO) which is $E_{\mathrm{th}}=29 \mu \mathrm{J} / \mathrm{cm}^{2}$. The lower threshold of BP was attributed to the structural similarity to PFO, improved purity and high gain of $g=$ $51 \mathrm{~cm}^{-1}$. (We will discuss in section 2.3 that polyfluorenes can exist in different morphologies, which allows substantial reduction of the ASE thresholds in PFO.) Lasing was demonstrated for BP spin-coated onto a 2-dimensional DFB grating and lasing thresholds of $4.5 \mu \mathrm{J} / \mathrm{cm}^{2}$ were achieved. 


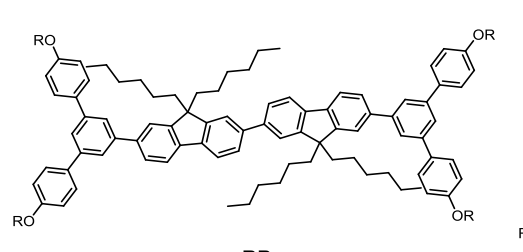

BP

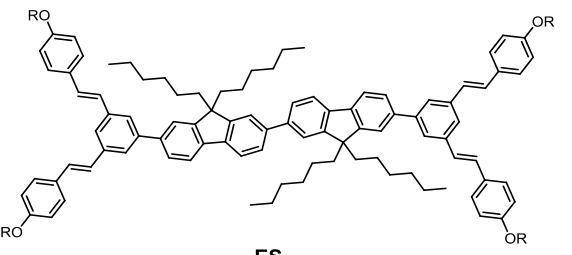

ES

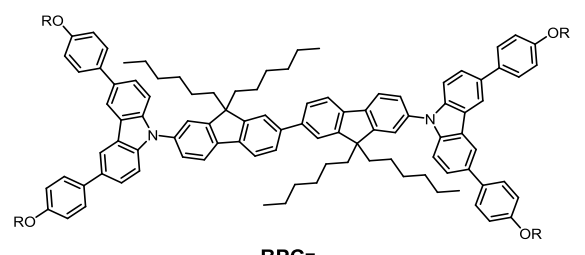

$\mathrm{BPCz}$

As will be discussed in the next section, spirobifluorene molecules that combine two perpendicular fluorene units in one molecule, offer facile functionalization of fluorene units. Their perpendicular arrangement allows for decisive synthesis of hetero-substituted molecules with precisely oriented donor and acceptor units.

\subsubsection{Towards orbital design via precise functionalization with donor and acceptor groups}

If the overlap between the HOMO and the LUMO orbital in a molecule is very small, the energy gap between the singlet $S_{1}$ and triplet $T_{1}$ state can be reduces to values on the order of $k T$. Under these circumstances, a substantial reverse intersystem cross rate from the $T_{1}$ state back to the $S_{1}$ state is expected at room temperature. Following conversion back to the S1 state, fluorescence can occur and this process is referred to as 'thermally activated delayed fluorescence' (TADF). In OLEDs TADF provides an alternative to phosphorescent emitters for harvesting the $75 \%$ of excitons with triplet character that are generated during electrical excitation ${ }^{41}$. The large fraction of triplets formed under electrical excitation also represents a major challenge on the way to electrically driven organic lasers. While the use of TADF emitters may be appealing in this context, the reverse intersystem crossing rates achieved so far appear to be too low and the triplet-triplet absorption too high to allow direct use of TADF molecules as gain materials. However, there is promising work on using these materials as co-dopants, as will be discussed (in section 6.3). ${ }^{42}$ 
During the early development stages of TADF, functionalization of spirobifluorenes with electron donating units on one hemisphere and acceptor units on the other was used to engineer a suitable configuration of frontier orbitals. DPA-SBF, for instance, is a spirobifluorene, which is functionalized with diphenylamine groups as donor moieties on one hemisphere and cyano groups as acceptor groups on the other. The frontier molecular orbitals are thus separated on the two perpendicular fluorene sites. Indeed, this molecule showed TADF and OLEDs based on DPA-SFB showed higher external quantum efficiency than would be expected for a conventional fluorescent emitter with the PLQY of DPA-SBF. ${ }^{43}$

Although the spirobifluorene motif is currently not the most attractive structure for TADF in OLEDs, it remains highly promising as a laser material. This is because it offers the ability for rational design of the overlap between HOMO and LUMO orbitals and generally shows high ASE performance. The carbazol functionalized spirobifluorene Cz-SBF, which resembles DPA-SBF but lacks the cyano moieties, shows clear separation of HOMO and LUMO orbitals to both hemispheres. Extension of the donor side by introduction of a connecting phenyl group (yielding CzP-SBF) led to a change in the electronic wave function density and to considerable orbital overlap. Extension with a phenylene-vinylene group (CzPVSBF) evoked a similar effect. Unlike their TADF counterparts, these molecules exhibited efficient stimulated emission and showed no significant decrease in fluorescence upon cooling (the fingerprint of TADF). A film of neat Cz-SBF had an ASE threshold of $25 \mu \mathrm{J} / \mathrm{cm}^{2}$, while the extended CzPV-SBF exhibited an ASE threshold of only $0.4 \mu \mathrm{J} / \mathrm{cm}^{2} .44,45$ When doped into a TPD derivative matrix at $6 \mathrm{wt} \%$, ASE thresholds down to $0.11 \mu \mathrm{J} / \mathrm{cm}^{2}$ were achieved for CZPV-SBF, which represents one of the lowest threshold reported for organic emitter to date. ${ }^{45}$ The carbazole units can also be replaced by diphenylamine groups, which allowed more rotational freedom of the individual phenyl rings. This resulted in a shift of the stimulated emission wavelength from $424 \mathrm{~nm}$ for Cz-SBF to $452 \mathrm{~nm}$ for TPA-SBF. The latter exhibited a temperature dependent ASE threshold between 1 and $2 \mu \mathrm{J} / \mathrm{cm}^{2}{ }^{26}$ The respective phenylenevinylene extended spirobifluorene with diphenylamine instead of carbazole had an ASE 
threshold of $2 \mu \mathrm{J} / \mathrm{cm}^{2} .{ }^{42}$ Such emitters can be combined with TADF molecules to recycle triplets (or at least prevent accumulation of molecules in the triplet state). This will be discussed in section 6.3.

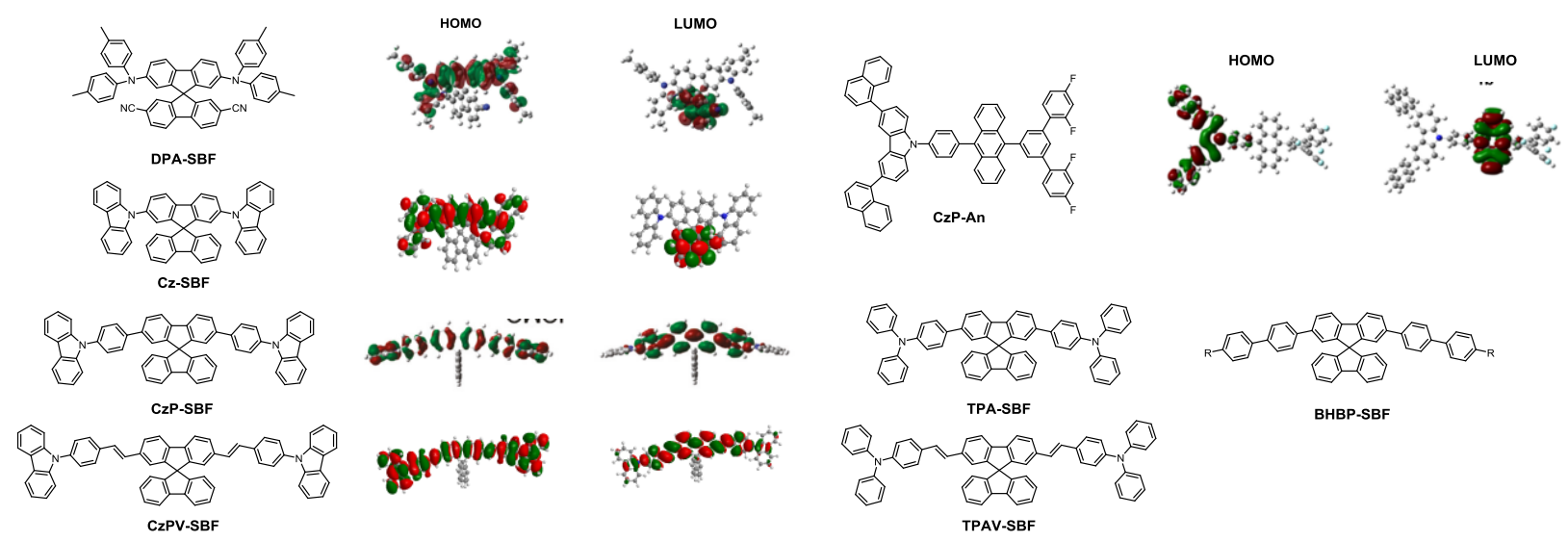

Molecular frontier orbitals calculated using DFT B3LYP functionals. Molecular frontier orbitals were taken from references (DPASFB). Reproduced in part from $\left[{ }^{43}\right]$ with permission of The Royal Society of Chemistry., CzP-SFB Reproduced from $\left[{ }^{44}\right]$. Copyrigh2011 American Chemical Society, CzP-An Reprinted from $\left[{ }^{47}\right]$, Copyright 2015, with permission from Elsevier) or were calucated by the authors of this review (for Cz-SFB and CzPV-SFB).

Spirobifluorenes without dedicated donor acceptor interplay were also investigated with regards to their laser performance. On DFB gratings bis-hexylbiphenyl spirobifluorene (BHBP-SBF) doped into PMMA exhibited lasing at $408.8 \mathrm{~nm}$ with a threshold of $42 \mu \mathrm{J} / \mathrm{cm}^{2}$.

Other molecular geometries exhibiting separation of the HOMO and LUMO distributions were also investigated. Asymmetrically substituted anthracenes are an important class of such molecules. Carbazole substituted anthracenes were found to exhibit orthogonal HOMO and LUMO distributions, as the anthracene twists out of plane with the carbazole. This effectively separates the HOMO and LUMO orbitals and minimizes the overlap integral. The molecules formed glasses and it was possible to process them onto DFB gratings via spin-coating. The laser emission was at $\lambda_{\text {las }}=460 \mathrm{~nm}$ and the lasing threshold was $5.7 \mu \mathrm{J} / \mathrm{cm}^{2} .{ }^{47}$

When comparing the molecules DPA-SBF, Cz-SBF and CzP-An, one finds that molecules with separated frontier orbitals appear to be either excellent laser gain media (CZ-SBF, CzP-An) or can be TADF active, in 
which case they seem not to be good gain media (DPA-SBF). Frontier orbital separation and minimization of the overlap integral on its own thus does not guarantee good TADF performance. Further research may yield materials, which combine both TADF and high gain. Alternatively, effective pairs of gain media and TADF materials can be of great interest ${ }^{42}$ to advance the recently re-sparked work towards electrically driven organic lasers, which remains the holy grail for many scientists working in this field.

\subsection{Defined star-shaped macromolecules}

Dendrimers and star-shaped oligomers are interesting materials as they combine the morphological characteristics of polymers (see section 2.3) with the ability to be purified like small molecules. Amorphous, liquid crystalline and supra-molecular arrangements can be obtained depending on the molecular architecture and the processing conditions and the materials can be purified by sublimation for smaller molecules - or by column chromatography for larger star-shaped molecules. (Both of these means of purification are not applicable for polymers and colloids.) As a result, compared to $\pi$ conjugated polymers, $\pi$-conjugated dendrimers exhibit higher PLQYs, fewer molecular impurities and improved photo-stability. A very substantial review article about star-shaped oligomers and their applications in organic electronics and photonics was published in 2010 and covers various conjugated and non-conjugated cores with arms of fluorenes, phenylenes, phenylene-vinylene, arylene-acetylene, thiophene and combinations of these. ${ }^{7}$ Here, we therefore limit the discussion to recently reported materials that have shown particularly promising laser characteristics.

An important class of star shaped oligomers are fluorene based materials with truxene cores. The truxene motif consists of three fluorene units, which share or overlap in one benzene ring. Truxenes with between one to four fluorene generations were compared to benzene cored star-shaped fluorene materials. ${ }^{48}$ In general, the ASE thresholds decreased with increasing number of generations and fluorene arm length. The truxene cored materials exhibited lower ASE thresholds $\left(T_{3}=2.1 \mathrm{~kW} / \mathrm{cm}^{2}\right)$ than 
the benzene cored materials $\left(B_{3}\right.$ and $\left.B_{4}=4.5 \mathrm{~kW} / \mathrm{cm}^{2}\right)$. The truxenes also exhibited much higher PLQY in solution and solid state, ranging for generations 1 to 4 between $78 \%$ and $87 \%$ while the spread was much larger in the benzene cored dendrimers (from $36 \%$ to $86 \%$ ).

The $T_{3}$ molecule was also used for further studies. To produce free standing and photo-lithographically patternable films, $T_{3}(20 \mathrm{mg} / \mathrm{mL})$ was incorporated into an aliphatic vinyl ether based photoresist. Diluted in this matrix, $\mathrm{T}_{3}$ exhibited an ASE threshold of $E_{\mathrm{th}}=400 \mu \mathrm{J} / \mathrm{cm}^{2}{ }^{29}$ By contrast, the pure $\mathrm{T}_{3}$ material had an ASE threshold of $8.4 \mu \mathrm{J} / \mathrm{cm}^{2}$ and a lasing threshold of $2.06 \mu \mathrm{J} / \mathrm{cm}^{2}$ with a very broad tuning range of $51 \mathrm{~nm}$ in the blue part of the spectrum. ${ }^{50}$ The pure $\mathrm{T}_{4}$ material exhibited an ASE threshold of $40 \mu \mathrm{J} / \mathrm{cm}^{2}$ in the pure state and a laser threshold of $2.7 \mu \mathrm{J} / \mathrm{cm}^{2}$ on 1- and 2-dimensional DFB gratings. ${ }^{51}$
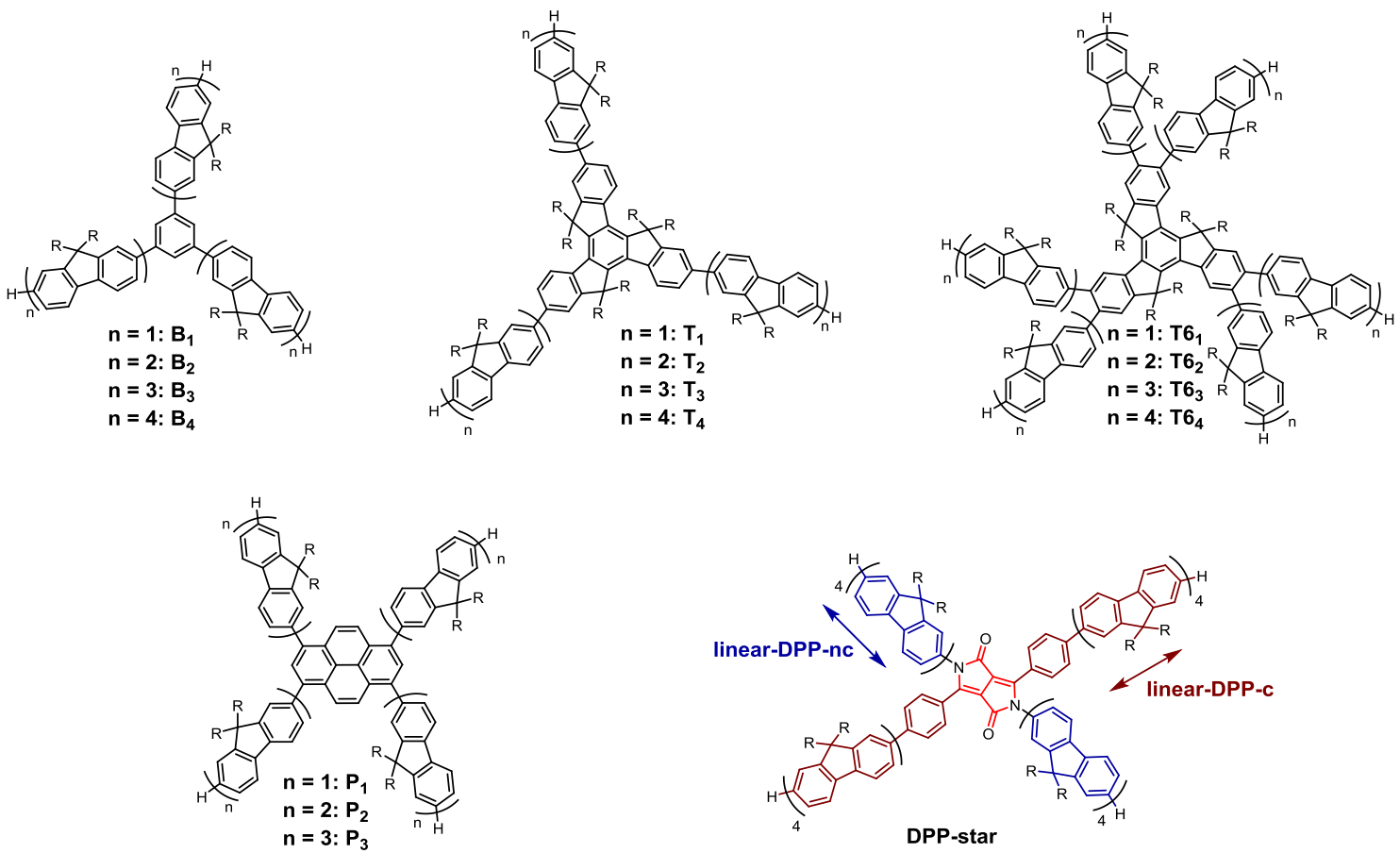

The concept can be extended by attaching six instead of three dendrons to the truxene core. The solid state PLQY of a third generation T6-truxene $\left(\mathrm{T}_{3}\right)$ was $70 \%$ and $\mathrm{T}_{3}$ exhibited tunable lasing on a 1dimensional DFB-grating. (The emission wavelength was tuned by adjusting the film thickness.) At $1.3 \mu \mathrm{J} / \mathrm{cm}^{2}$ the laser threshold is very similar to the 3 -armed truxenes. ${ }^{52,53}$ 
The concept of star-shaped dendrimers lends itself very well to a bio-mimetic approach. Following the strategy of bacterial absorption cascade photosynthesis, the arms of the molecule can be used as absorbing antennas that transfer the absorbed energy to the core of the molecule. One example is the functionalization of pyrene and diketopyrrolopyrrole (DPP) cores with four fluorene arms. ${ }^{54,55}$ While the pyrene-cored dendrimer $\left(\mathrm{P}_{1-3}\right)$ is fully $\pi$-conjugated over the entire molecule, the DPP-cored star (DPPstar) has a conjugated and a non-conjugated axis. To understand the electronic effects involved, linear polymers with the DPP unit in the conjugated orientation (linear-DPP-c) and the non-conjugated orientation (linear-DPP-nc) were produced as well. While the fluorene arms worked as efficient antennas, resulting in a PLQY of over $90 \%$ for the $\mathrm{P}_{2}$ and $\mathrm{P}_{3}$ dendrimers, the DPP star and linear polymers exhibited only low PLQY of up to $6 \%$. When the DPP-star was excited in a spectral region where the DPPcore showed strongest absorption, a PLQY of up to $20 \%$ was achieved. This indicates a rather inefficient energy transfer from the fluorene arms to the DPP core, most likely due to insufficient energetic (and spectral) overlap of the DPP-core with its fluorene arms. The absorption of the pyrene core, by contrast, overlaps well with the emission of the fluorene arms, thus facilitating efficient energy transfer to the core in this molecule. High gain and low laser thresholds were obtained for the $\mathrm{P}_{2}$ and $\mathrm{P}_{3}$ molecules $(g=$ $\left.78 / 75 \mathrm{~cm}^{-1} ; E_{\mathrm{th}}=0.15 / 0.26 \mu \mathrm{J} / \mathrm{cm}^{2}\right)$. In the DPP cored molecule no spectral narrowing was observed; however, the linear-DPP-c polymer exhibited spectral narrowing (indicating the presence of ASE), in particular when the DPP core was excited directly (threshold excitation density, $42 \mu \mathrm{J} / \mathrm{cm}^{2}$ versus $106 \mu \mathrm{J} / \mathrm{cm}^{2}$ when pumping the fluorene arms).

\subsection{Linear Macromolecules}

$\pi$-conjugated fluorescent polymers are produced from a variety of monomers and can range from highly defined and sequence controlled to randomly composed chains. Polymers exist in a variety of morphologies, ranging from completely amorphous to highly crystalline. While the former usually show good optical characteristics, the latter often exhibit better charge transport characteristics. Polymers 
exhibit attractive mechanical properties and excellent film forming properties and thus they are highly attractive for solution-based fabrication of flexible electronic and photonic devices.

An important class of polymers in the context of organic lasers are polyfluorenes and their co-polymers. Polyfluorene can adopt various metastable morphologies and the exact morphology depends on the thermal history of the material and/or on the fabrication process. A large body of literature exists on the various phases and photo-physical characteristic of polyfluorenes with different side chains. Poly(9,9dioctylfluorene) (PFO) is probably most widely known and exhibits a particularly rich phase behavior. The lowest lasing thresholds amongst the fluorene polymers were observed for the $\beta$-phase morphology, in which individual polymer chains adopt an extended $\pi$-conjugation without inter-molecular alignment. More recent studies concentrated on the refractive index difference between the $\beta$-phase and the amorphous phase of polyfluorene, which allowed generation of interesting device geometries featuring both morphologies. The $\beta$-phase of PFO showed an additional absorption shoulder at lower energies ( $\sim 30 \mathrm{~nm})$ compared to the amorphous morphology. This led to a 1-2\% increase in refractive index in this spectral range. DFB lasers based on spin-coated layers of PFO in $\beta$-phase morphology showed a bathochromic shift of the $\beta$-phase laser line by a few nanometers versus a DFB laser based on the amorphous material. ${ }^{56}$

Different morphologies of PFO can be introduced via thermal cycling, spin-coating from various solvents and exposure to solvent vapors. Liquid crystalline phases were also induced by deposition of the PFO onto rubbed polyimide layers. ${ }^{57}$ A DFB grating was imposed on the PFO layer via nanoimprinting. If the orientation of the PFO chains is controlled with respect to the grating direction of the nanoimprinted 1dimensional DFB resonator, the wavelength of the laser emission and the laser threshold can be tuned. In this way, the effective refractive index of the material can be adjusted to the resonator geometry and laser thresholds as low as $20 \mu \mathrm{J} / \mathrm{cm}^{2}$ were achieved. At the same time, alignment of the polymer 
improved charge carrier mobility. Being able to improve these two properties simultaneously is of great interest for electrically pumped lasing as described in section 6.2.

More recently and to combine both the amorphous and the $\beta$-phase morphology in one device, PFO was spin-coated onto a grating in the amorphous phase and then exposed to toluene vapor through a mask. In this way, different morphologies were induced side by side on one grating. ${ }^{58}$ In a different approach, PFO was functionalized with vinyl ether moieties in the alkyl periphery. These polymers can be crosslinked amongst each other using UV-initiated photoacid generators (PAG) or electron beam lithography to form an insoluble network. ${ }^{59,60} \mathrm{~A}$ particular advantage of this strategy is that $\beta$-phase induced in these polyfluorenes can be fixed by crosslinking. This allows subsequent solvent processing without dissolving the crosslinked PFO film and affecting the morphology pattern. Using this strategy, a DFB grating was defined into a $\beta$-phase crosslinkable VE-PFO using electron beam lithography followed by spin-coating of an amorphous layer of PFO on top, yielding a layered mixed morphology device. Such a stack of amorphous and $\beta$-phase material emitted laser radiation from both morphologies at the same time if the excitation densities exceeded the threshold of both. In the $\beta$-phase morphology and for a well-tuned grating, the crosslinkable VE-PFO material exhibited laser thresholds as low as $0.5 \mu \mathrm{J} / \mathrm{cm}^{2}$. In a related approach, polysilafluorenes were equipped with vinyl and epoxide containing side groups. Using a PAG, which decomposed into an acid and an undefined amount of radical species, these side groups were photo-chemically crosslinked and films of the polymer were patterned using photolithography. On a 1-dimensional DFB grating, crosslinked films of this polymer exhibited lasing between 430 and $450 \mathrm{~nm}$; however, no lasing thresholds were reported. ${ }^{61}$

PFO can also be pumped in 2- and 3-photon absorption processes. When excited with sufficiently short pulses, organic materials show two photon absorption cross-sections. Nano- and femto-second pulses with intensities in the gigawatt per $\mathrm{cm}^{2}$ range in the red spectral range were used to pump a PFO film on a 1-dimensional DFB grating and tunable laser emission between 440 and $460 \mathrm{~nm}$ was observed. ${ }^{62}$ A 3- 
photon process was used to pump an oligofluorene that was end-capped with diphenylamine and triazole as electronic donor-acceptor system to enhance polarizability. In this example, femto-second pulses from a near-IR laser were used to induce up-converted deep blue laser emission. Oligomers with a larger number of fluorene units showed better performance than those with lower molecular weight. ${ }^{63}$ While lasing thresholds for multi-photon pumping have so far been much higher than for single-photon pumping (due to the relatively low two photon absorption cross-section), multi-photon pumping may still be beneficial in the future as a way of reducing photo-oxidative stress to the material.

To improve the charge carrier mobility in polyfluorenes, dioctylfluorene was copolymerized with diethylhexylfluorene. A homo-polymer consisting of only dioctylfluorene (PFO), and polymers with 4:1 and 1:1 ratios of dioctylfluorene and diethylhexylfluorene (PFO-EH) were synthesized. While ASE and laser wavelength were around $450 \mathrm{~nm}$ for all polymers, the lowest lasing threshold $\left(0.3 \mu \mathrm{J} / \mathrm{cm}^{2}\right)$ was obtained for the 4:1 ratio. At the same time, this material exhibited the highest hole mobility (determined by time-of-flight, TOF) amongst these materials $\left(\mu_{\mathrm{h}}=370 \times 10^{-4} \mathrm{~cm}^{2} /(\mathrm{Vs})\right){ }^{64}$ The increased mobility is thought to result from improved interchain charge hopping at the ethylhexyl functionalized monomer sites, where the proximity between polyfluorene chains is greater than at the octyl sites, which isolate the polyfluorene chains.

Looking at other polyfluorene co-polymers, poly(dioctylfluorene-co-benzothiadiazole) (F8BT) is a wellknown yellow-green emitting conjugated polymer with acceptable lasing performance $\left(28 \mu \mathrm{J} / \mathrm{cm}^{2}\right)^{65}$. By changing the ratio of fluorene (F8) to benzothiadiazole (BT) units from 1:1 to 9:1 the gain of this material was greatly improved as shown by an ASE threshold as low as $2 \mu \mathrm{J} / \mathrm{cm}^{2}$. This improvement was attributed to enhanced chain isolation through the octyl side chains effectively preventing exciton annihilation processes. ${ }^{66}$ To enhance and control the molecular microstructure, fluorene units were bridged with methylene groups to force neighboring fluorene units into conjugation. Different persistence lengths were induced using molecular motifs with predetermined conjugation, from the non- 
bridged polyfluorene via poly(indenofluorene) (PIF) and higher poly(indacenofluorenes) (PIAF) to the fully bridged and rod-like ladder-type poly(p-phenylenene) (PPP). PIF with 3 bridged phenyl rings (or two overlapping fluorene units) showed the lowest ASE threshold and highest gain. ${ }^{67}$ Inspired by this study, indenofluorene co-polymers were investigated further. Poly(indenofluorene-co-phenanthrene) (PIF-PhA) was found to have an ASE threshold close to that of the PIF homopolymer $\left(4.5 \mu \mathrm{J} / \mathrm{cm}^{2}\right.$ and $2.8 \mu \mathrm{J} / \mathrm{cm}^{2}$, respectively). ${ }^{68}$ However, PIF-PhA exhibited very high optical gain of up to $g=154 \mathrm{~cm}^{-1}$. PIF-PhA also showed a TOF hole mobility of $\mu_{\mathrm{h}}=10^{-2} \mathrm{~cm}^{2} /(\mathrm{Vs})$. When tested in an OFET geometry, which is often argued to be the more relevant device setup for organic lasers, hole mobilities up to $0.5 \mathrm{~cm}^{2} /(\mathrm{Vs})$ were obtained. These examples of simultaneous optimization of laser performance and charge carrier mobility represent important steps on the long route towards electrically pumped lasers (see section 6.2).

A second important class of linear macromolecules besides the fluorene-based polymers are poly(phenylenevinylenes) (PPVs). The most widely used PPV is known under the trade name 'Super Yellow' and still is sometimes used as a gold standard for polymer based OLEDs. For organic lasers, terphenylene vinylene polymer (BBEHP-PPV) is more relevant due to its high gain. Lasing thresholds as low as $0.04 \mu \mathrm{J} / \mathrm{cm}^{2}$ were reported and BBEHP-PPV was used in an organic laser based sensor to detect explosives. ${ }^{69}$ In other work, BBEHP-PPV was mixed with CHDV at $10 \mathrm{mg} / \mathrm{mL}$ and spin-coated onto a DFB grating. Diluted in this transparent matrix, BBEHP-PPV exhibited a lasing threshold of $1.1 \mathrm{~mJ} / \mathrm{cm}^{2}{ }^{70}$ BBEHP-PPV was also diluted by adding monodisperse silica spheres. These can assemble into a photonic glass and act as a resonator for the BBEHP-PPV gain polymer. Random lasing was observed at thresholds around $3 \mathrm{~mJ} / \mathrm{cm}^{2} .{ }^{71}$ In a different approach, silica and titania nanoparticles were used to produce a 1dimensional vertical cavity DFB device which was infiltrated with PPV. Using two-photon pumping, this device was excited with $180 \mathrm{fs}$ pulses at $780 \mathrm{~nm}$ and laser emission from PPV was observed at $530 \mathrm{~nm}$ for excitation fluxes above $150 \mu \mathrm{J} / \mathrm{cm}^{2}{ }^{72}$ Poly(2-methoxy-5-(2'-ethyl-hexyloxy)-1,4-phenylene vinylene) was infiltrated into an aligned mesoporous silica template with $\sim 5 \mathrm{~nm}$ pores, allowing the arrangement of a 
few polymer chains per pore with perfectly parallel alignment. ${ }^{73}$ The confinement led to polarized ASE with thresholds twenty times lower than for non-oriented polymer samples. Furthermore, ASE depended on the polarization of the incoming excitation beam with respect to the orientation of the pore/polymer chain direction.

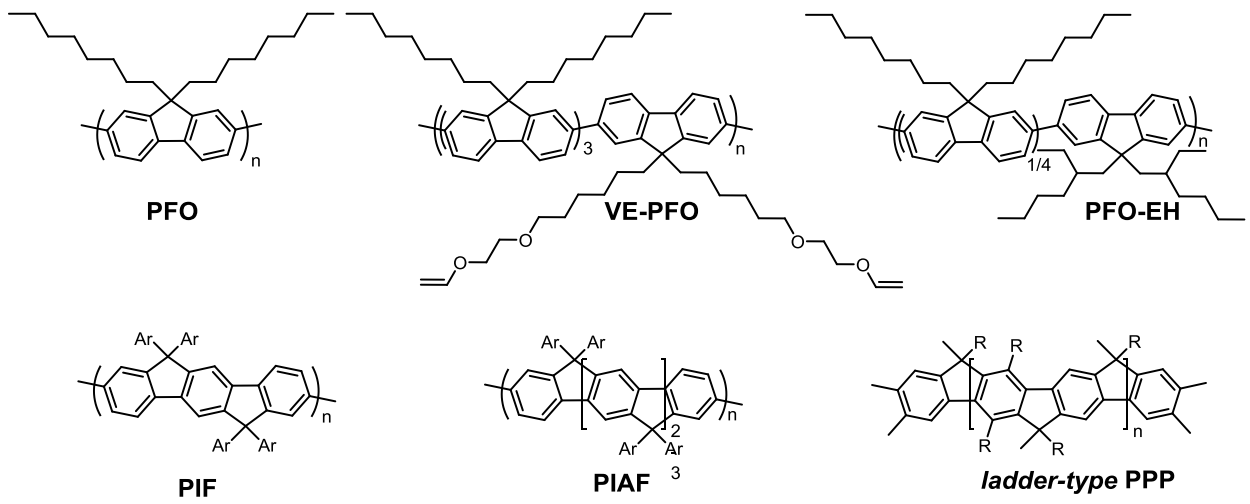

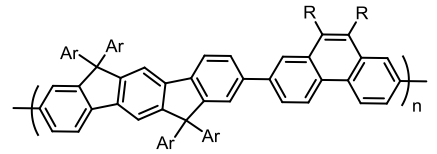

PIF-PhA
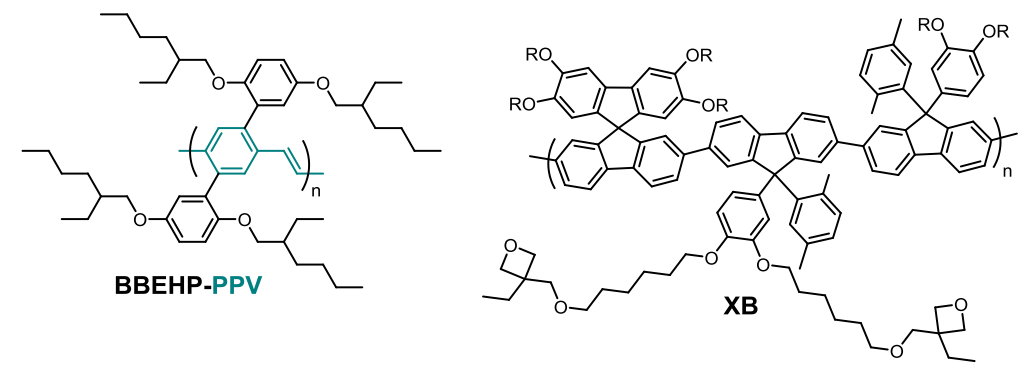

Lasing was also observed in ternary and higher co-polymers. Binary blends of poly(hexylthiophene) and F8BT were compared to the copolymer poly(fluorene-co-benzothiadiazole-co-thiophene-cotriphenylamine) which is known as Red F. ${ }^{74}$ Mixing of RedF into F8BT allowed dilution of the dithiophene benzothiadiazole (DTBT) emitter unit and prevented unproductive concentration quenching. ${ }^{75}$ Using this strategy, thresholds were reduced by up to six-fold, to $44 \mu \mathrm{J} / \mathrm{cm}^{2}$, when $30 \%$ RedF was incorporated in an F8BT matrix. Similarly, blending of poly(hexylthiophene) into F8BT prevented quenching from stacked polythiophene segments and facilitated ASE and lasing $\left(E_{\mathrm{th}}=26 \mu \mathrm{J} / \mathrm{cm}^{2}\right)$ from polythiophene. Ternary and higher spirobifluorene copolymers also facilitated tuning of the emission wavelength across the entire visible spectrum. ${ }^{76}$ In particular, the BT unit as well as a DTBT and other units, including hole-conducting TPD units, were incorporated into the polymer. Amongst these were also polymers that were 
functionalized with oxetane units and these were used to define multilayer structures via photocrosslinking as explained above for VE-PFO above. The crosslinked polymers exhibited the same ASE threshold fluences as non-oxetane functionalized polymers with otherwise identical backbone structure and aliphatic, non-crosslinkable side groups. ASE thresholds were in the range of 4.4 to $10 \mu \mathrm{J} / \mathrm{cm}^{2}$ for green and red emitting polymers and in the $\mathrm{mJ} / \mathrm{cm}^{2}$ range for the blue emitting polymers. Lasing was also obtained for a spirofluorene-co-dibenzothiophenedioxide polymer (25-35\% dibenzothiophenedioxide). Using a 1-dimensional DFB grating structure, the emission wavelength was $\lambda_{\text {laser }}=475 \mathrm{~nm}$ and thresholds were similar to other fluorene-based polymers. ${ }^{77}$

Various approaches were pursued to control the intermolecular interactions with the goal of adjusting charge-carrier mobility and optical gain characteristics independently, which in the long-run may overcome current limitations for electrically pumped lasers. Remaining drawbacks and further strategies towards improved lasers that go beyond material optimization on its own are discussed in section 6 .

\subsection{Biological Dyes and Fluorescent Proteins}

Fluorescent proteins may represent the class of fluorescent molecules, in which humans first observed fluorescence although their official discovery only dates back to $1962 .^{78}$ Even more recently, fluorescent

proteins have also been applied as laser gain medium. ${ }^{79,80}$ The most well-known fluorescent protein is the green fluorescent protein (GFP), a polypeptide macromolecule naturally found in the Aequorea Victoria jellyfish. The $27 \mathrm{kDa}$ protein comprises a much smaller fluorescent moiety, which forms on oxidative coupling of the three amino acids Tyr, Gly, and Ser. This luminophore is situated at the center of the protein molecule inside a so called beta-barrel structure (essentially a peptide nano-cylinder). The beta-barrel effectively shields and protects the luminophore from the environment. Recently, it was shown that this prevents concentration-quenching in GFP even if molecules are present in their solidstate form, a property that renders GFP highly attractive for laser applications. ${ }^{81}$ 
By extracting a short segment of DNA from Aequorea Victoria and introducing it as a transgene into other species, one can program nearly any organism - from bacteria to higher mammals-to produce GFP. Fluorescent proteins with other emission colors can be obtained by mutation of the original DNA, which leads to shortening (blue) or extension (yellow, red) of the $\pi$-conjugated emitter system. In the first demonstration of a laser based on fluorescent proteins, a cell line based on human embryonic kidney (HEK) cells was programmed to produce eGFP (a genetically enhanced GFP variant) and these cells were placed between two dielectric mirrors. Upon pumping with ns-pulsed blue light, lasing was observed in the green part of the spectrum. ${ }^{79}$ E. coli bacteria programmed to produce GFP also showed lasing in biofilms $\mathrm{s}^{80}$ and when dispersed in microdroplet cavities ${ }^{82}$.

In addition to using fluorescent biomolecules inside cells, they can also be useful to generate lasing outside their biological hosts. For instance, thin films of eGFP and other fluorescent proteins were used as laser materials in planar micro-cavities and to form self-assembled ring-resonators lasers by exploiting the 'coffee-stain effect'. The sensitivity of the optical gain of fluorescent proteins to local humidity levels was used for sensing. ${ }^{81}$ Förster resonance energy transfer (FRET) between green and red emitting fluorescent proteins has been used to shift the emission wavelength of optofluidic protein lasers. ${ }^{83}$ Lasing has also been achieved using single molecular layers of fluorescent proteins. ${ }^{84}$ eGFP based lasers were also used to analyze complex mode interactions in planar micro-cavities. ${ }^{85}$ Most recently, it was shown that the special molecular structure of fluorescent proteins enables polariton lasing under nanosecond pumping (ps or shorter excitation was so far required for other synthetic organic materials, see section 7.1). ${ }^{86}$

Small biological fluorescent molecules can also be applied as laser gain media. Luciferin, the bioluminescent molecule in fireflies, bacteria and marine species have been applied in optofluidic lasers. When a luciferin solution is pumped through a glass micro-capillary, whispering gallery mode lasing can 
be obtained at $E_{\mathrm{th}}=7.6 \mathrm{~mJ} / \mathrm{cm}^{2}$. The large Stokes shift in luciferin is believed to avoid undesirable reabsorption effects. which may be important for ultra-sensitive sensing applications. ${ }^{87}$

Riboflavin (vitamin B2) is another fluorescent biomolecule that was used for lasing. It has a molecular weight of $457.36 \mathrm{Da}$ and emits in the green part of the spectrum. Ribovlavin was explored in a variety of resonator structures, including Fabry-Pérot, whispering gallery mode droplet resonators formed on a superhydrophobic surface, micro-droplets in a poly-L-lactic acid micro-well and films on a DFB gratings. ${ }^{88,89}$ Typical laser thresholds of riboflavin droplets and riboflavin coated DFB gratings were around $1 \mathrm{~mJ} / \mathrm{cm}^{2}$ and $18 \mathrm{~mJ} / \mathrm{cm}^{2}$, respectively.

\subsection{Perovskites}

Perovskite materials have recently received a lot of attention by members of the organic electronics community. Initially the interest was mostly focused on applications in photovoltaics where very promising results have been achieved in terms of power conversion efficiency. More recently, there has also been work on lasers based on perovskite materials. Per definition perovskites are inorganic materials; however, they share some qualities with organic materials and can easily be interfaced with organic electronic and photonic devices. It is therefore worthwhile to review some of the recent advances in perovskite based lasers. In contrast to inorganic III-V semiconductors, perovskites can be produced not only by vapor deposition but can also be readily obtained by wet chemical processing. The recent wave on perovskite activity in optoelectronics mostly evolves around alkyl ammonium leads halides, which are produced from precursors - an alkyl ammonium halide and a lead dihalide salt. Such perovskite precursor mixtures could be spin coated from DMSO solutions, which stabilized crystal seeds, prevented further crystallization and thus yielded stable precursor solutions. The actual perovskite was produced upon deposition and baking of the material. ${ }^{90}$ Baking is performed at moderate temperatures, usually below $100^{\circ} \mathrm{C}$, which makes perovskites compatible with organic materials. Nonetheless, for 
optimal performance the interfaces between organic layers and the perovskite need to be engineered, as roughness and interface chemistry can induce crystallization and direct crystal orientation of the perovskite. ${ }^{91,92}$ It was shown that the emission color of perovskites can be tuned by varying the halide composition from $\left(\mathrm{MeNH}_{3}\right) \mathrm{PbCl}_{3}$, over $\left(\mathrm{MeNH}_{3}\right) \mathrm{PbBr}_{3}$ to $\left(\mathrm{MeNH}_{3}\right) \mathrm{APbl}_{3}$ and that mixed halide perovskites can have emission ranging across the entire visible spectrum (from $400 \mathrm{~nm}$ up to $~ 800 \mathrm{~nm}$ ). ${ }^{93}$ More bulky ammonium components (with bulky group R) were shown to shift the composition to ( $R$ $\left.\mathrm{NH}_{3}\right)_{2} \mathrm{PbX}_{4}$, which allows further tuning of the electronic bandgap and emission into the UV regime. ${ }^{94}$ Perovskites have generally much higher charge carrier mobilities than organic materials, which results from their crystalline nature and large crystalline domains with few grain boundaries in the film. ${ }^{95-97}$ This feature makes perovskites interesting laser materials, especially in the context of the continuing search for simple, tunable and solution-processable electrically pumped lasers.

$\mathrm{MeNH}_{3} \mathrm{Pbl}_{3}$ perovskites were examined for their ASE behavior and thresholds of around $10 \mu \mathrm{J} / \mathrm{cm}^{2}$ were reported. ${ }^{98}$ The low ASE threshold was attributed to the high absorption coefficient $\left(5.7 \times 10^{4} \mathrm{~cm}^{-1}\right.$ at $600 \mathrm{~nm}$ ), the low density of bulk defects and slow Auger recombination. Optically pumped lasing from perovskites was achieved in a variety of different geometries: One example is based on a perovskite layer sandwiched between a distributed Bragg reflector (DBR) and a gold film. As exposure to oxygen causes decomposition of perovskites and deterioration of their opto-electronic performance, a thin PMMA layer was inserted between the gold and the perovskite layer for protection. Using this approach line narrowing was observed for a mixed $\mathrm{Cl} / \mathrm{I}$ perovskite at a fluence of $0.2 \mu \mathrm{J} /$ pulse. ${ }^{99}$ To produce whispering gallery mode (WGM) lasers (see section 3 for optical feedback geometries), $\mathrm{MeNH}_{3} \mathrm{Pbl}_{3}$ was deposited onto silica spheres with diameters of $>50 \mu \mathrm{m}$ using an atomic vapor deposition process. Thresholds of $65 \mu \mathrm{J} / \mathrm{cm}^{2}$ were obtained for ASE and lasing thresholds of $76 \mu \mathrm{J} / \mathrm{cm}^{2}$ were determined for the whispering gallery resonator. ${ }^{100}$ Perovskite micro-crystals were also used to directly achieve WGM lasing in green and in the NIR $(\sim 80 \mathrm{~nm})$ with thresholds of $11 \mu \mathrm{J} / \mathrm{cm}^{2}$ and $37 \mu \mathrm{J} / \mathrm{cm}^{2}$, respectively. ${ }^{101,102}$ In addition, 
lasing was observed for nanowires with mixed halide compositions and emission lines from 500 to $800 \mathrm{~nm}$ were obtained. Nanowires were produced in a surface initiated growth strategy, where a solid film of lead acetate was brought in contact with a highly concentrated isopropanol solution of the methyl

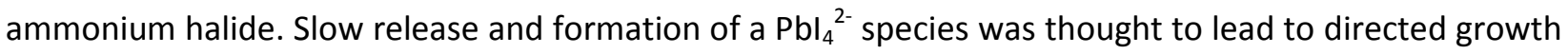
following the two following equations:

$$
\begin{aligned}
& \mathrm{PbAc}_{2} \text { (solid) }+4 \mathrm{I}^{-} \text {(solution) } \rightarrow \mathrm{Pbl}_{4}{ }^{2-} \text { (solid) }+2 \mathrm{Ac}^{-} \text {(solution) } \\
& \mathrm{Pbl}_{4}{ }^{2-} \text { (solution) }+\mathrm{MeNH}_{3}{ }^{+} \text {(solution) } \rightarrow \mathrm{MeNH}_{3} \mathrm{Pbl}_{3} \text { (nanowire) }+\mathrm{I}^{-} \text {(solution) }
\end{aligned}
$$

The nanowires had end facets with widths of a few hundred nanometers and lengths of up to $20 \mu \mathrm{m}$. Lasing was obtained at low thresholds, down to $0.22 \mu \mathrm{J} / \mathrm{cm}^{2}$, and the laser activity was attributed to the nanowires acting as Fabry-Pérot resonators (see sections 3.1 and 3.5 for details on this optical feedback geometry). ${ }^{103}$

\subsection{Conclusion}

There have been a number of exciting developments on materials for organic lasers over the last decade. Small molecules that form their own resonator upon crystallization have improved in terms of laser thresholds. Although the crystalline state of the molecules may be expected to be detrimental to lasing due to enhanced self-quenching, this issue can now be managed for certain molecular designs. The constraints of a crystal lattice can then lead to materials with improved stability against photo-induced degradation. However, finding the optimal balance between dense molecular packing and reduced selfquenching through intermolecular separation of $\pi$-systems remains a challenge. The unique nanostructure of biologically produced fluorescent proteins, which has evolved in a multi-million year process, may provide important guidance in this context.

Over the years organic gain materials were improved and optimized for their laser performance in various ways. In earlier studies the development of new materials was to some extent serendipitous with 
improvements made in a trial and error fashion. By careful analysis of the most potent laser materials available, the community then began to develop methods for morphology control so that the crystalline and amorphous character of the materials can now be tuned. In more recent years scientists have focused on the synthesis of molecularly precise architectures and on materials with specific intermolecular and electronic interactions, leading to further advances in terms of threshold, gain and photo-stability.

Perovskites may also well play a role in future organic/inorganic hybrid laser devices due to their relatively low laser thresholds, high absorption coefficient and high charge carrier mobilities.

The recent development of molecules with carefully designed frontier orbitals led to highly efficient small molecule emitters that offer some of the lowest ASE thresholds reported to date (see Figure 2). Furthermore, the development of triplet converting TADF molecules that can be used as or in conjunction with low threshold emitters may help to overcome the challenge of triplet accumulation and thus improve the chances of achieving CW operation and electrical pumping in organic lasers. (While most TADF materials reported to date are of low molecular weight - typically mixed into a small molecule or polymer host - there are already first reports on polymeric materials supporting TADF. ${ }^{104}$ )

It is intriguing to see that fluorene based materials remain amongst the most powerful laser materials, offering the lowest ASE- and lasing thresholds. It is highly promising to see that further improvements to the performance of fluorene homopolymers can be made by fine tuning the fluorene moiety into to indenofluorene, phenanthrene or by variation of the alkyl periphery. 


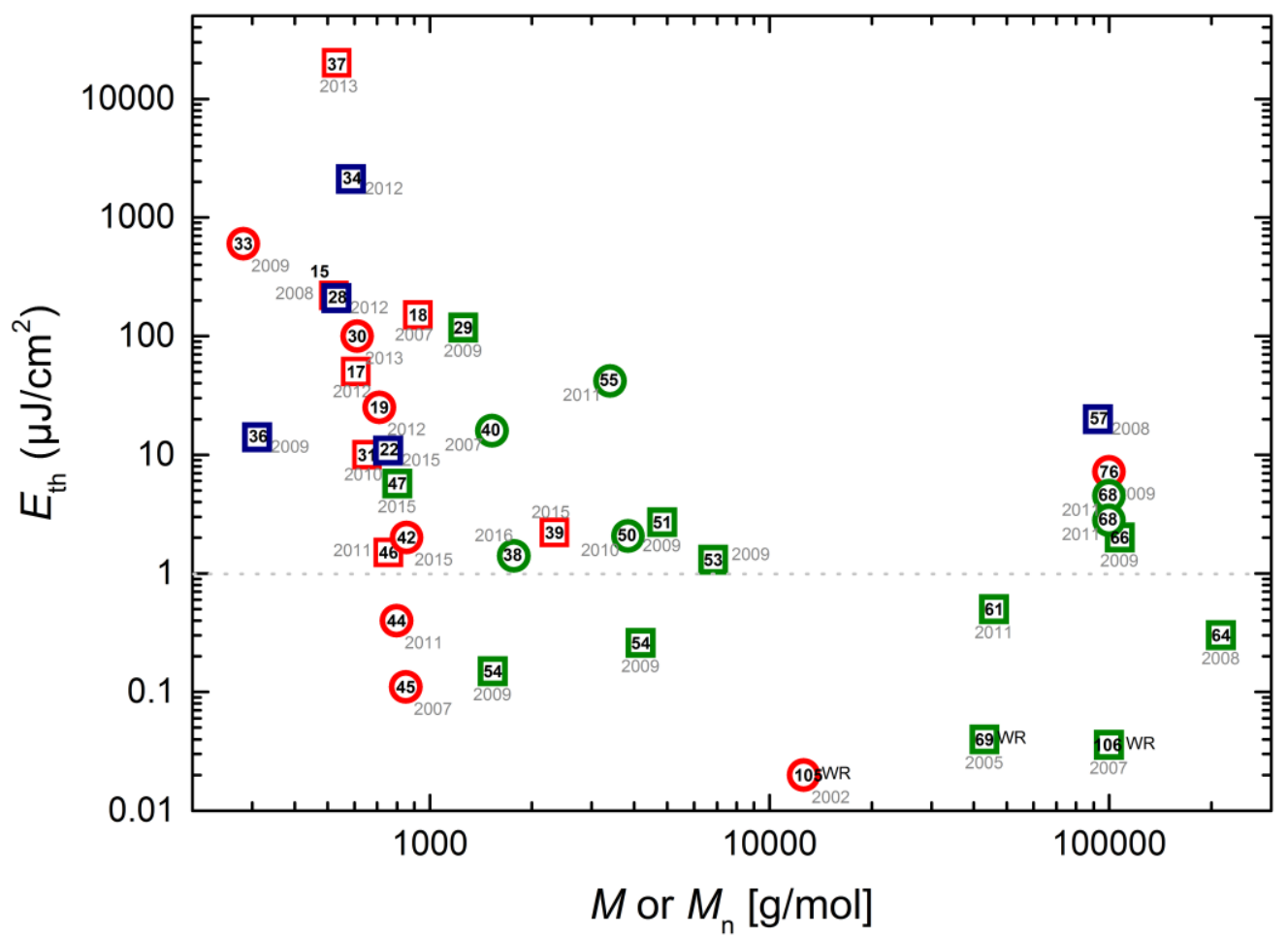

Figure 2: Thresholds for ASE and lasing of organic gain materials reported since 2007 versus molecular weight of the material. Three "world record" low thresholds from before 2007 are also included, marked with WR ${ }^{69,105,106}$. Round data points represent ASE thresholds and square data points represent laser thresholds. Red data were obtained from host guest systems, green data from glassy and blue data from single crystalline specimen (except for reference [57], which is liquid crystalline).

This leads to the exciting question of whether generally applicable structure property relations exist that allow identifying molecular structures that facilitate particular low ASE and lasing thresholds. Unfortunately, there is no straightforward answer to this question, as the lasing threshold depends on both the material and the optical feedback structure used (feedback structures will be discussed in the next section). In addition, ASE thresholds depend on film thickness, substrate quality and measurement configuration, which makes them difficult to compare between labs. Due to a wealth of different potential applications and interests, the community has so far unfortunately not been able to adopt a standard configuration to characterize organic materials with regard to their lasing performance. 
From the data reported in the literature, one empirically finds that the materials, which have shown the lowest ASE or laser thresholds $\left(<1 \mu \mathrm{J} / \mathrm{cm}^{2}\right.$, Fig. 2), are mostly based on fluorene units such as spirobifluorene, ${ }^{44,45}$ star-shaped molecules with fluorene $\operatorname{arms}^{54}$ and poly(fluorene)s ${ }^{61,64,106}$ or on fluorene-vinylene-phenylene-vinylene copolymers ${ }^{105}$ and poly(phenylene-vinylene)s ${ }^{69}$. In addition, it appears that for all systems tuning of the intermolecular distance and random molecular orientation or amorphous morphology is crucial for obtaining low laser thresholds. This non-ordered molecular morphology can be achieved by dispersion of the gain medium into dielectric host materials, which is often done with small molecules to prevent crystallization and aggregation or by carefully designing the peripheral side groups of macromolecular gain media. The individual molecules need to be separated far enough to prevent self-quenching but when considering electrically pumped lasing (see section 6) separation can be detrimental to charge carrier mobility. ${ }^{64,69}$

The thresholds listed in Fig. 2 are given in terms of pump fluence (i.e. in $\mu \mathrm{J} / \mathrm{cm}^{2}$ ). When this measure is used, the best laser performances are generally achieved with resonators based on the DFB geometry, with mixed order DFB gratings (for efficient feedback and outcoupling) resulting in the lowest thresholds among these ${ }^{106}$ (section 3.2.1). When instead looking at the absolute threshold pump pulse energy required to reach the lasing threshold, more compact planar or whispering gallery mode resonators reach the most attractive values. To explore the influence of structure, the following section will therefore review the different optical feedback structures that are used by the community and explain their advantages and disadvantages.

\section{Optical Feedback Structures}

A plethora of different concepts for providing optical feedback in lasers has been developed since the initial demonstration of lasing in the 1960. Most of these designs have also been applied to organic lasers. Although the general resonator geometry and the underlying physics are similar for organic and 
inorganic lasers, the special characteristics of organic gain materials provide distinct opportunities for optimizing the optical feedback structure. Therefore, it is important to discuss recent developments in the field of optical feedback structures for organic lasers. The following section will review the main resonator structures that are considered for organic lasers, again with a strong focus on developments reported over the last decade. The most common resonator structures are summarized in Figure 3.

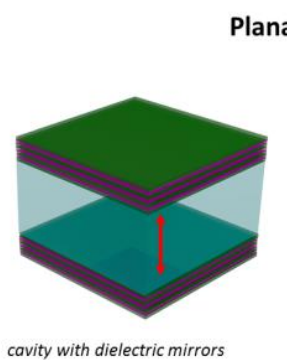

cavity with dielectric mirrors

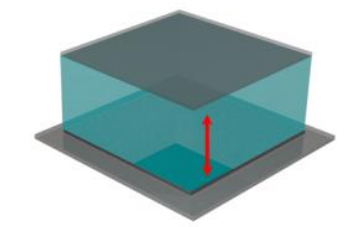

cavity with (semitransparent) metal mirrors

Whispering Galleries
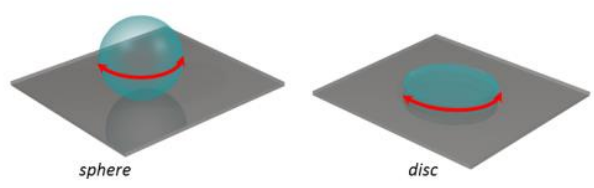

Photonic Crystals

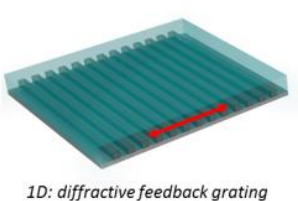

1D: diffractive feedback grating

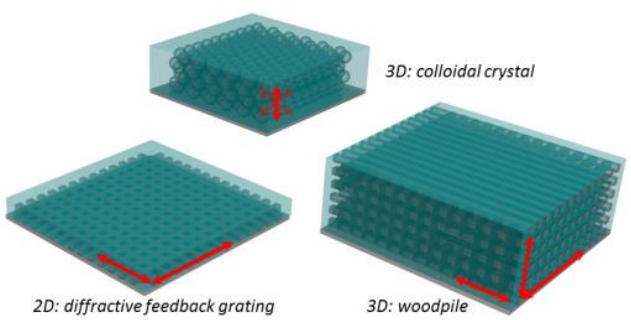

Single Crystals

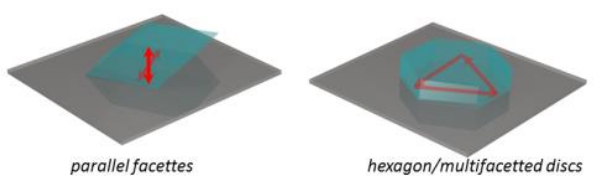

Figure 3: Resonator structures used for organic lasers: Planar cavities are sandwich-type, layered structures with the gain medium between a pair of reflective mirrors. At least one of the mirrors is partially transparent in the spectral range where the gain medium emits to allow light extraction. Photonic crystals rely on diffractive feedback by highly periodic grating structures. There are one, two and three-dimensionally patterned structures. In whispering gallery mode resonators, feedback is created by repeated or continuous reflection within a circular resonator. Organic gain media can also be crystallized into facetted single crystals with planar faces. Depending on the geometry, the crystals themselves can act as the resonator.

Although the use of an organic gain medium would generally be considered sufficient for a laser to qualify as 'organic laser', there is an increasing number of examples where both the gain medium and the optical feedback structure are 'organic'. In many cases, the feedback structure is in fact formed by the gain material itself. Such approaches are advantageous in terms of fabrication cost and mechanical flexibility. Yet, many of the most efficient organic lasers continue to be based on feedback structures produced from inorganic or non-emissive organic materials interfaced with an organic gain material. 


\subsection{Planar cavities}

Perhaps the most obvious structure for an organic laser is a planar cavity comprising two highly reflective mirrors sandwiching the organic gain material (Figure 3). These Fabry-Pérot optical micro-cavities were among the first to be investigated for generating laser emission from organic semiconductors ${ }^{107,108}$. The mirrors of organic micro-cavity lasers can be either formed by thermally evaporated metal layers or by DBR mirrors. The limited reflectance of the former often prevents efficient laser operation, in particular for thin cavity structures with optical thickness of a few hundred nanometers to a few microns (i.e. only a few multiples of $\lambda / 2$ ). By contrast, DBR mirrors, which are typically formed by alternating layers of two oxides with different refractive index, provide much higher reflectance (often above 99\%) enabling organic micro-cavity lasers with extremely low absolute threshold pulse energies (down to < $100 \mathrm{pJ} /$ pulse). ${ }^{81}$ The deposition of a DBR mirror usually requires electron beam evaporation or sputtering and this can lead to issues with degradation of the organic material underneath.

Micro-cavity structures are well suited for manipulating and studying the mode structure of organic lasers. This can be easily achieved by varying the thickness of the cavity along one axis of the sample. By moving the excitation spot along this axis, the laser wavelength can then be changed. ${ }^{109}$ Over the past decade, the introduction of lateral structures into the micro-cavity has become increasingly popular. These structures can provide lateral confinement of light and thus reduce lasing thresholds. As the emission from micro-cavity lasers is usually confined to a small area, one can readily investigate the influence of these lateral geometries on the mode structure, e.g. by recording spectrally resolved maps of the in-plane momentum of cavity photons (energy dispersion) ${ }^{110}$ or by taking hyperspectral images of the spatial emission profile ${ }^{79}$ (Figure 4). These methods have been applied for example to study microcavities containing 'photonic dots ${ }^{111,112}$, dielectric gratings ${ }^{110}$, metallic gratings ${ }^{113}$, perforated metal layers $^{114}$, ring structures of biologically produced fluorescent protein material ${ }^{85}$ or even the emission from biological cells embedded in a micro-cavity ${ }^{79,115,116}$. 
(a)

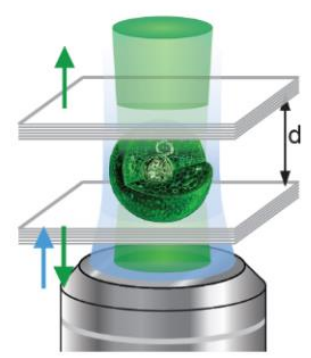

(b)

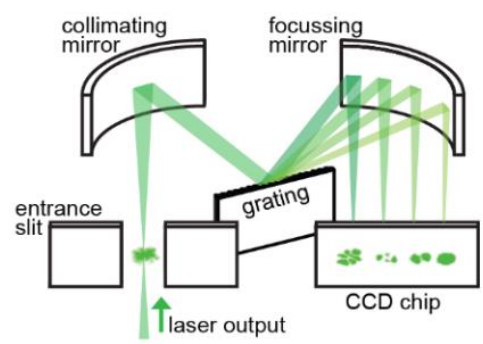

(c)
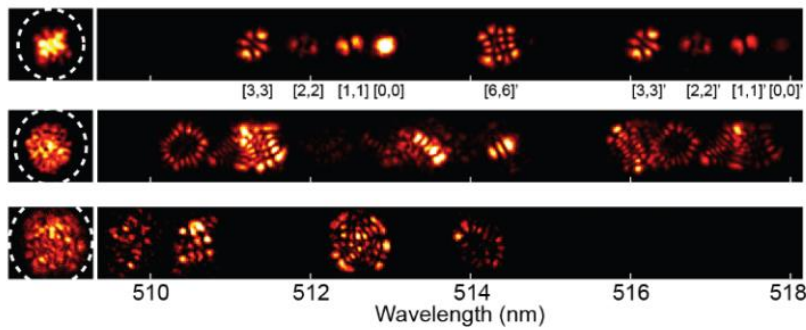

(d)

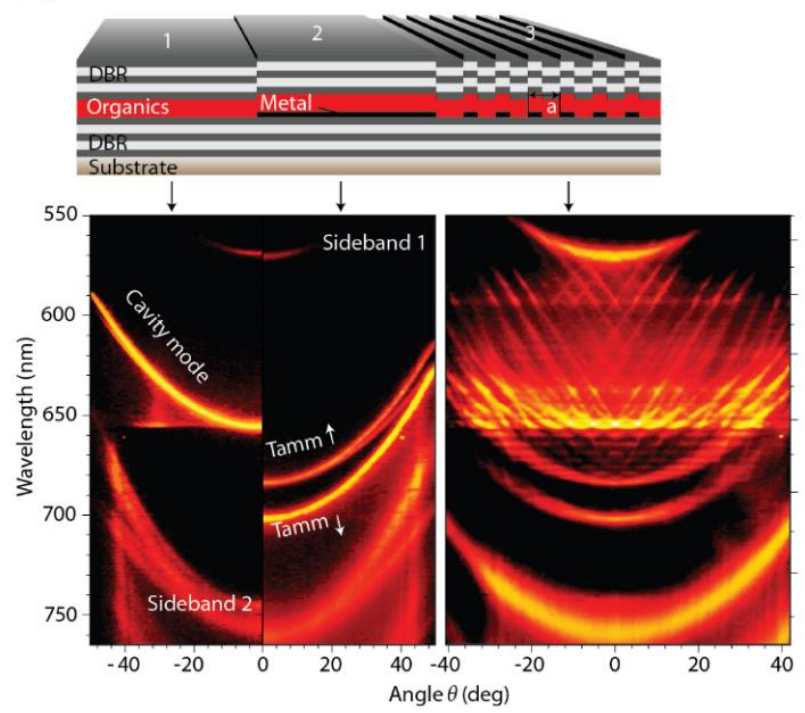

Figure 4: Organic lasers based on planar cavities are well suited for manipulating and studying their mode structure. (a) Planar cavity laser based on single live cell expressing the green fluorescent protein as optical gain medium ${ }^{79}$. (b) Schematic of hyperspectral imaging setup and (c) resulting mode analysis for three representative single cell lasers, showing on the left the spatial emission pattern of the laser and on the right the spectrally separated spatial patterns ${ }^{79}$. (d) Top: Schematic of plain organic micro-cavity, 1 , and micro-cavity with embedded metal layer, 2 , or metal grating, 3 . The presence of the metal gives rise to hybrid states resulting from coupling of plasmonic Tamm modes and cavity modes. Bottom: Momentum space imaging of the cavity emission allows direct investigation of the dispersion of mode in the different cavities. ${ }^{113}$ Reprinted by permission from Macmillan Publishers Ltd: Nature Photonics [ $\left.{ }^{79}\right]$, copyright 2011 and $]^{113}$ ], copyright 2012.

Micro-cavity structures supporting strong coupling between the excitons present in the organic material and the cavity photons have recently enabled room-temperature exciton-polariton condensation in organic materials. ${ }^{86,117-119}$ These exciton-polariton condensates are discussed again in section 7.1. 
In most organic micro-cavity lasers, the organic material is in an amorphous or semi-crystalline state. However, it has also been proposed to sandwich single-crystalline materials between DBR mirrors. One advantage of this approach would be the ability to align all transition dipole moments of the material in the plane of the cavity, which would result in better coupling of emitted photons to the cavity modes $^{120,121}$. There are also a number of reports on organic lasers where the micro-cavity itself is formed by organic crystals; these are discussed in section 3.5 .

Instead of defining the micro-cavity with metallic mirrors or with DBR mirrors made from oxides, one can also use two polymers with different refractive index to form DBR mirrors. Besides simplifying device fabrication, this approach also helps to avoid damage to the organic gain material, which is often considered an issue for sputter deposition of oxide DBRs. Recent work in this area has focused on efficient melt-extrusion of such micro-cavity lasers ${ }^{122}$ and on improving DBR reflectivity by optimizing the refractive index contrast of the used polymers ${ }^{123}$.

Over the past decade, a number of planar cavity structures with macroscopic cavity lengths, i.e. many wavelengths in optical thickness, have also been used for organic lasers. In one example, a disposable glass slide coated with a dye doped polymer film was inserted into a macroscopic external cavity ${ }^{124}$. Very recent work demonstrated that such external cavity lasers can be driven with a pulsed blue-emitting laser diode and that slope efficiencies in excess of $10 \%$ and output pulse energies of up to $280 \mathrm{~nJ}$ can be achieved with this approach ${ }^{125}$. External cavity lasers also allowed introduction of additional intra-cavity optical elements, e.g. for frequency doubling (Figure 5) ${ }^{126}$. Wavelength tuning was also be achieved by adjusting the thickness of the film forming the gain medium and by using this film as an intra-cavity etalon. ${ }^{127} \mathrm{~A}$ different form of macroscopic cavity organic laser was based on self-written active waveguides that were fabricated within the gap between the end facets of two optical fibers ${ }^{128}$. 


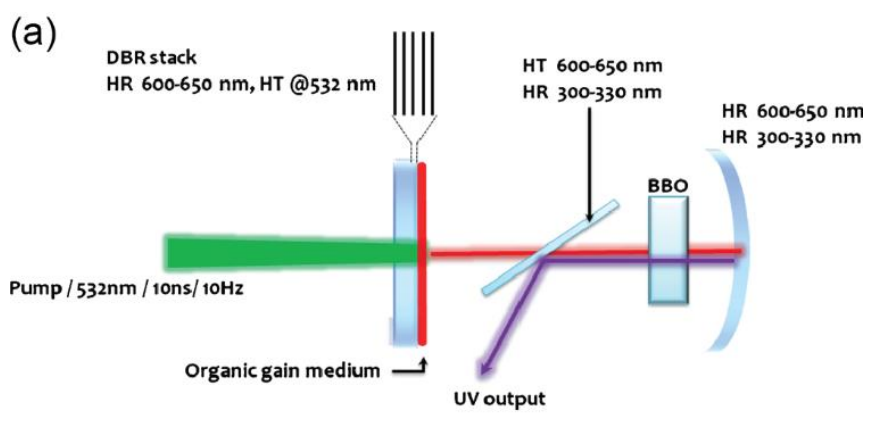

(b)
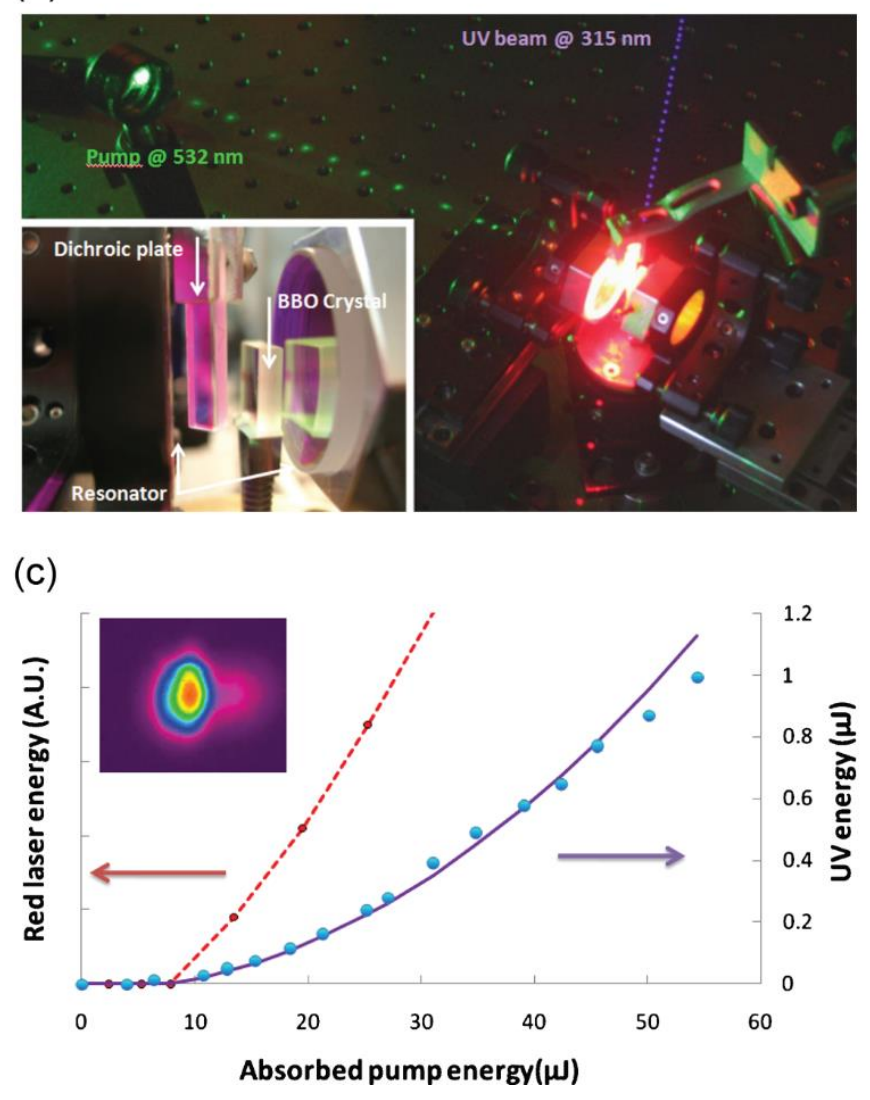

Figure 5: External cavity organic lasers. (a) Schematic and (b) photograph of a configuration with frequency doubling intracavity optics for generation of UV light. (c) Input-output characteristics for fundamental red and frequency doubled UV emission from laser, with beam profile of UV beam shown as inset. Reprinted from $\left[{ }^{126}\right]$ with the permission of AIP Publishing.

In most micro-cavity lasers, the optical axis of the cavity is perpendicular to the plane of the substrate and the mirrors are layered onto the substrate. There are however also planar micro-cavity structures. For these the mirrors are often formed by gratings, and due to their structural similarity to DFB lasers, they will be discussed together with these in section 3.2. An interesting alternative to this approach is to 
define an in-plane reflector by a line of sub-wavelength sized cylinders ${ }^{129}$. When two of these lines are placed a few $\mu \mathrm{m}$ apart and the structure is filled with a fluorescent polymer, lasing can be obtained under sub-ps pumping.

For many applications, real-time tuning of the lasing wavelength is of great interest and there have been reports on how this can be achieved for many resonator configurations. For planar micro-cavities, this can be achieved by direct electrostriction if one of the two cavity mirrors is mechanically flexible. This was realized for example by a thin suspended membrane that deflects when an electric field was applied. With this approach the laser wavelength was tuned over a $10 \mathrm{~nm}$ range by applying a voltage of just $6 V^{130}$

The micro-cavity geometry is also highly relevant for potential electrically driven organic laser diodes. This is in part due to its similarity to the standard OLED device configuration, which means that one can benefit from the relatively mature OLED technology. Nevertheless, important technical challenges remain. In order to from highly reflective cavity mirrors, one usually has to resort to oxide-based DBR mirrors but their deposition on an OLED stack may damage the organic material underneath. In addition, electrodes need to be inserted into the cavity to deliver sufficient current to the OLED stack sandwiched between the mirrors but the optical absorption of these electrodes can cause a substantial reduction in the $Q$-factor of the cavity. This reduction in $Q$-factor often prevents lasing entirely, not only under electrical pumping but also under optical pumping. One approach to overcome this limitation is the introduction of metal gratings into a DBR cavity. When designed carefully, these gratings did not prevent built-up of coherence in the cavity ${ }^{113,114}$ and may in the future be used for current injection with low Ohmic loss. Other recent work (re)visited the effect of introducing a transparent conductive oxide (TCO) layer and thin metal films into DBR based micro-cavities ${ }^{131}$ and also looked at fabricating high- $Q$ microcavity OLEDs in a configuration where a DBR mirror is only required underneath the OLED stack ${ }^{132}$. 


\subsection{Distributed feedback and photonic crystal structures}

\subsubsection{One dimensional DFB structures}

Distributed feedback (DFB) structures are another highly popular configuration for organic lasers. In these, light usually propagates in a slab waveguide in the plane of the sample. The substrate or the waveguide core contains a periodic corrugation, which scatters guided modes so they can recombine coherently. For certain wavelengths this leads to constructive interference and can thus provide the optical feedback required for laser action. A large number of reports on highly efficient, low threshold

DFB lasers were published in the early 2000s. These have been reviewed in detail in Refs. $\left.{ }^{3,10}\right]$, together with the general operation principle of organic DFB lasers. In the following we therefore focus on developments reported over the last decade. These were mostly aimed at reducing lasing thresholds further to facilitate optical pumping by low cost laser diodes and LEDs and also looked at simplifying device fabrication, developing versatile sensors based on DFB lasers, and modifying the device geometry to bring direct electrical pumping into reach.

While the absolute pump energy required to achieve lasing is often comparable to other designs with smaller mode volumes (such as the micro-cavity geometry discussed in the previous section), the threshold pump fluence (i.e. pulse energy per area) and threshold power density in optically pumped organic DFB lasers can be exceptionally small. This is a direct consequence of the fact that in most DFB configurations the resonator and the mode are spread out in the plane of the substrate. The low threshold flux of DFB lasers is advantageous for optical pumping because it reduces photo-oxidative stress exerted on the material. It also simplifies the use of poorly collimated or un-collimated light sources for optical pumping. Following a series of initial demonstrations of lasing from organic DFB lasers pumped by pulsed laser diodes ${ }^{133-135}$ the next milestone was to realize lasing with incoherent LEDs as pump sources. An important step in realizing this was the optimization of so-called mixed order DFB gratings. These combine a first order grating for optimal in plane feedback with a second grating that 
facilitates efficient outcoupling. Using this approach, absolute lasing thresholds of down to $45 \mathrm{pJ}$, threshold fluxes of $36 \mathrm{~nJ} / \mathrm{cm}^{2}$ and threshold power densities of $4 \mathrm{~W} / \mathrm{cm}^{2}$ were reported ${ }^{106}$. By combining these geometrical advances with improvements in organic gain materials and high-brightness blue LEDs, it became possible to achieve lasing in an organic DFB structure using pulsed excitation from an incoherent InGaN LED source ${ }^{136}$. While the initial demonstration was based on a grating structure defined lithographically in a silica substrate, it was recently shown that LED pumping can also be achieved with UV nano-imprinted DFB lasers structures (Figure 6) ${ }^{137,138}$. The latter geometries are simpler and cheaper to produce, which may be important in the context of laser based sensing applications (see section 4.3). Another recent study describes improvements in interfacing LED pump sources efficiently with organic DFB lasers (in terms of geometry and polarization) and how LED driving can be optimized. ${ }^{139}$ 
(a)

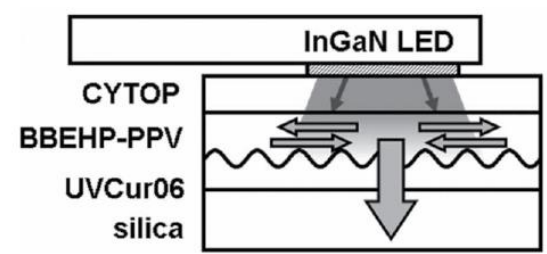

(b)
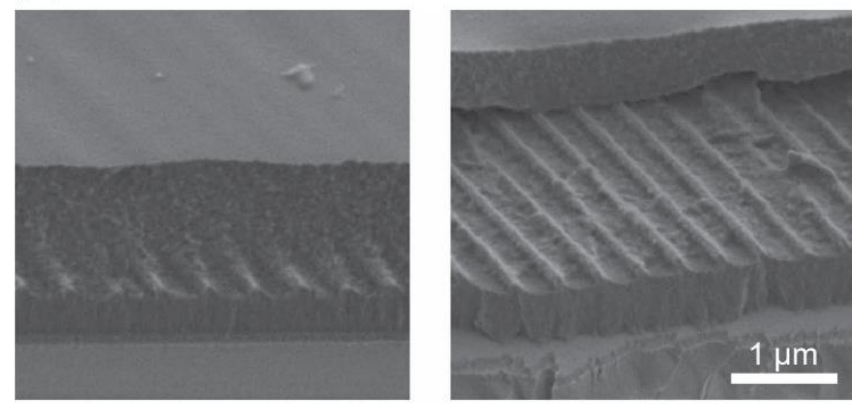

(c)

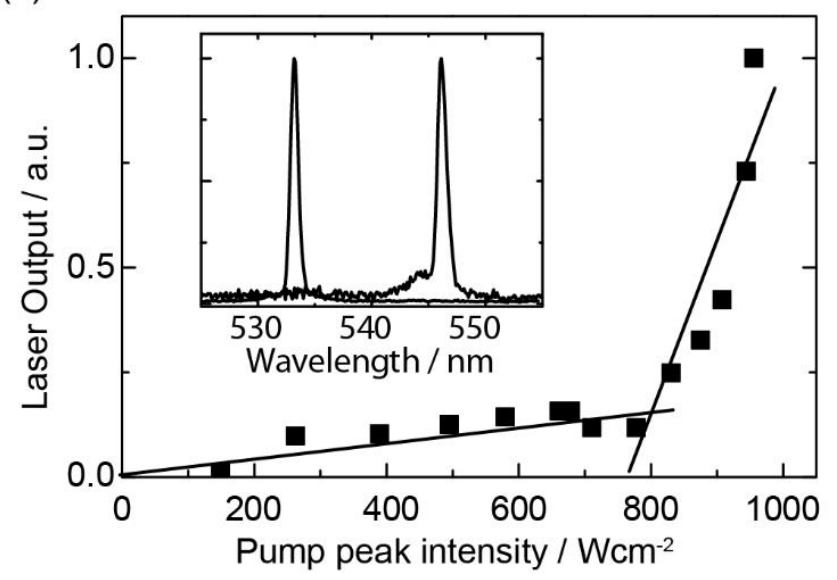

Figure 6: Organic lasers based on a distributed feedback structure pumped by an inorganic LED. (a) Schematic of the device architecture with organic laser on a silica substrate mounted onto an InGaN based LED. (b) SEM images of a cross-section of the nanoimprinted grating structure (left) and of the bare grating, i.e. with the gain polymer layer peeled back (right). (c) Inputoutput characteristic of the laser with threshold at around $800 \mathrm{~W} \mathrm{~cm}{ }^{-2}$. Inset shows lasing spectrum for two different devices with different active layer thickness. Reproduced with permission from $\left.{ }^{137}\right]$. Copyright 2013, Wiley-VCH Verlag GmbH \& Co. KGaA.

Despite dramatic improvements in lasing thresholds, efforts to optimize the geometry parameters of DFB lasers continue. Recent experimental work on second order DFB gratings looked in more detail at the dependence of grating depth on the laser wavelength and on the shape of the emission spectrum ${ }^{140}$. There were also efforts to simplify modelling of DFB lasers, e.g. to predict the lasing wavelength for multi-layer DFB structures ${ }^{141}$. 
A variation of the mixed order grating design introduced above are so-called 'sub-structured gratings'. These are second order gratings with two different grooves in their unit-cell. Such a grating effectively provides both first and second order components and by careful tuning of the width and position of the groves, the balance between the two can be adjusted. ${ }^{142}$ DFB lasers have also been stacked on top of each other to facilitate dual wavelength operation, in one example even using two different organic gain materials. This allowed simultaneous operation in the blue and red part of the spectrum. ${ }^{15}$

The fact that the laser wavelength not only depends on the grating period but through the effective refractive index of the supported mode also on the thickness of the different layers in the structure can be exploited for wavelength tuning. In one example, a DFB laser with a tuning range of about $35 \mathrm{~nm}$ was obtained by depositing a gain layer with a thickness gradient on a holographically produced DFB grating and moving the excitation spot along the substrate ${ }^{143}$. Similar devices were also obtained by solution processing, forming the thickness gradient through dip-coating of either a conjugated polymer or a blended small molecule film. In this case optical pumping was achieved with a diode laser. ${ }^{144}$ Even larger tuning ranges were achieved by introducing a high index layer of $\mathrm{Ta}_{2} \mathrm{O}_{5}$ with variable thickness into the device stack $^{145}$.

The laser wavelength can also be tuned by modifying the grating period in real time, e.g. if the grating is defined in an elastomeric substrate. For gratings defined by nano-imprint in poly(dimethylsiloxane) (PDMS) and coated with F8BT a $20 \mathrm{~nm}$ tuning range was achieved and lasing thresholds of $6.1 \mu \mathrm{J} / \mathrm{cm}^{2}$ were obtained under 150 fs pumping. In other work, the DFB gratings were defined by self-wrinkling that occurred after surface oxidization of a pre-stretched PDMS layer (Figure 7a-c). ${ }^{146}$ In addition, arrays of DFB lasers with different grating periods were defined within a common mechanically flexible substrate and tuning of laser wavelength by mechanical flexing was again observed. ${ }^{147}$ Very recently, the concept was extended further by inscribing DFB gratings into shape memory materials. This facilitated a permanent adjustment of the laser wavelength by non-elastic mechanical stretching of the substrate 
(Figure 7d-e). Thermal annealing above the switching temperature initialized a recovery of the original laser wavelength. ${ }^{148}$ 

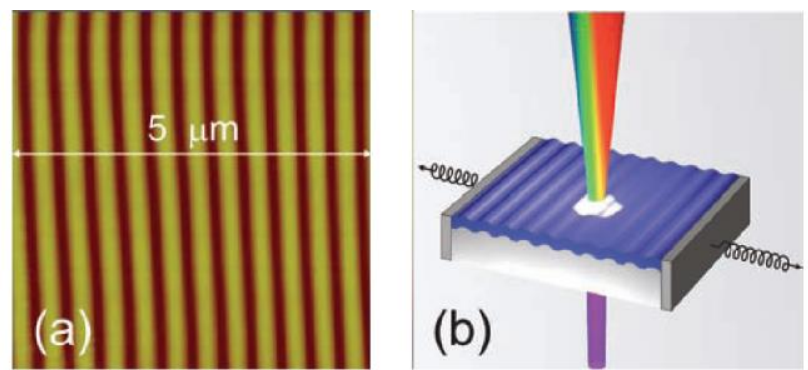

(c)

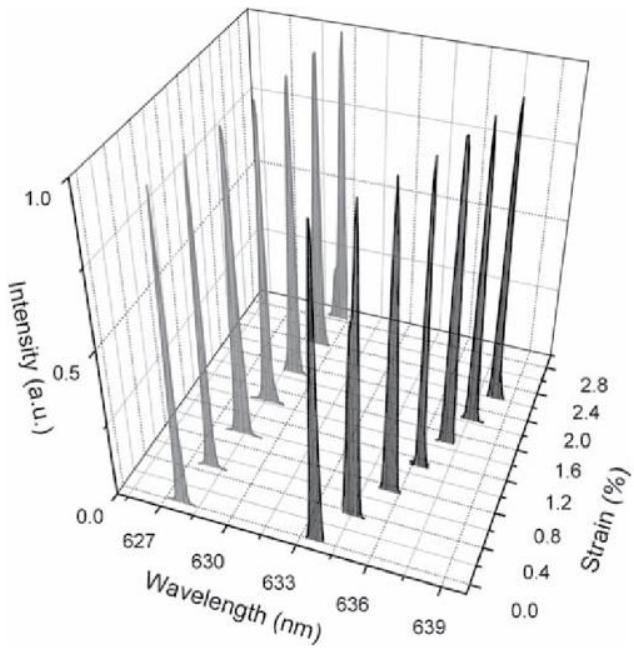

(d)

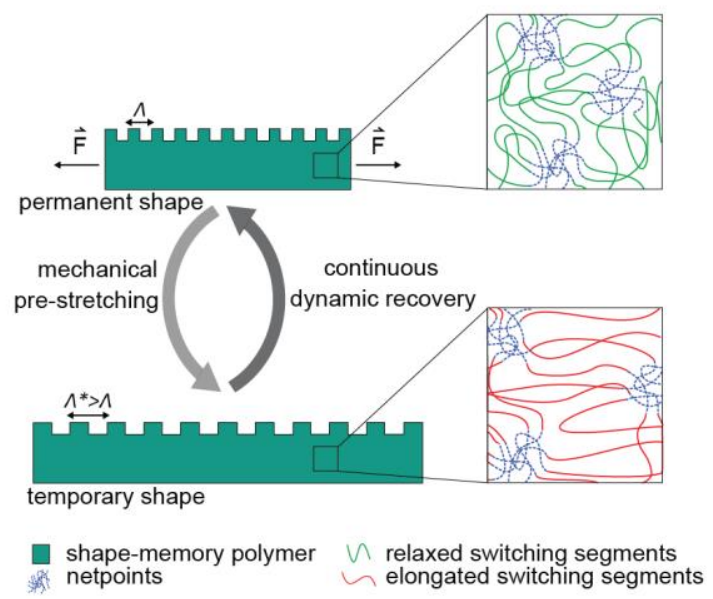

(e)

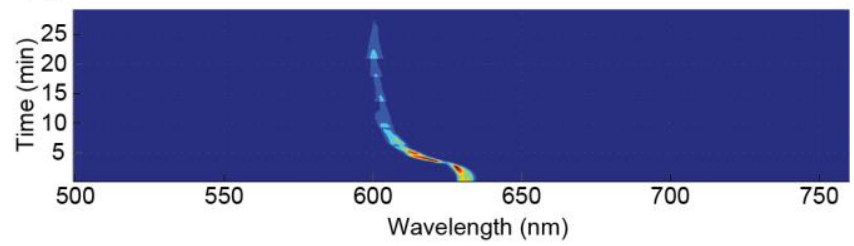

Figure 7: By depositing organic lasers on a deformable substrate, the wavelength of emission can be tuned mechanically. (a) Self-assembled grating structure formed by oxidization-induced surface wrinkling of a siloxane-based elastomer. ${ }^{146}$ (b) Schematic illustration of the laser tuning process. ${ }^{146}$ (c) Emission spectra of an organic DFB laser on a siloxane substrates for different levels of substrate strain. ${ }^{146}$ (d) Using shape-memory polymers, the laser wavelength can be adjusted, locked in and later recovered to its original value. ${ }^{148}$ (e) Representative change of lasing wavelength for a shape-memory polymer based DFB laser. ${ }^{148}$. Reproduced with permission from $\left[{ }^{146}\right]$. Copyright 2011, Wiley-VCH Verlag GmbH \& Co. KGaA. 
For most organic DFB lasers the feedback structure is defined by a corrugation of the substrate or of an additional non-emitting dielectric within the structure. An alternative configuration is to directly modulate the refractive index or thickness of the organic gain layer. In recent reports this has been achieved by controlled introduction of a periodic arrangement of polymer domains in $\beta$-phase conformation $^{60}$ and by using photo-crosslinkable fluorescent polymers and holographic patterning ${ }^{149}$ (see section 4 for more details).

\subsubsection{Two and three dimensional photonic crystal structures}

Most of the DFB structures discussed above restrict the propagation of light only in one direction within the plane of the sample (and prevent propagation out of the plane by the slab-waveguide geometry). By using 2-dimensional grating structures, one can restrict propagation in both directions within the substrate plane. 2 -dimensional photonic crystals with particularly small footprint $\left(\sim 30 \times 30 \mu \mathrm{m}^{2}\right)$ have been reported but thresholds were in the $1 \mathrm{~mJ} / \mathrm{cm}^{2}$ range (under $800 \mathrm{ps}$ excitation) and in the $40 \mu \mathrm{m} / \mathrm{cm}^{2}$ range when similar structures were pumped with 120 fs pulses ${ }^{150,151}$.

2-dimensional photonic crystal structures show rich dispersion characteristics, i.e. the emission intensity and wavelength change with angle of observation. Traditionally this has been measured by mounting the laser on a goniometer but increasingly single shot reciprocal space imaging setups are being used. This method can be applied either to look at emission/fluorescence or at the transmission and reflectance of a device. This 'Fourier spectroscopy' has also be applied to determine the quality factor of the modes supported by a DFB structure. ${ }^{152}$

Coupled wave theory is currently among the most popular approaches to model the modes in DFB structures. For 2-dimensional structures, a number of refinements were reported over recent years, in particular with respect to modelling different polarizations and describing the modes in square, rectangular and triangular lattice geometries ${ }^{153-157}$. 
The number of reports published over the past decade on truly 3-dimensional photonic crystal structures for lasing is rather limited. Inverse opal structures of zirconia were made in a sol-gel process using opals of sintered polystyrene beads as templates. The inverse opals where then backfilled with solutions of Rhodamine dyes in ethylene glycol. These structures showed lasing when excited at $\sim 4 \mathrm{GW} / \mathrm{cm}^{2}{ }^{2}{ }^{158}$ Similar inverse opal structures were also made from silica. In this case, the refractive index contrast between the silica structure and the backfilled ethylene glycol solution is rather low $(\Delta n \approx 0.04)$ and the authors of this study have suggested that feedback is due to gain modulation rather than due to refractive index modulation. ${ }^{159} \mathrm{~A}$ more recent example of a 3-dimensional photonic crystal structure is based on a self-assembled nanostructure. Here, blue-phase liquid crystals self-assembled into a 3dimensional periodic lattice with a diamond-like structure. This structure was then used as a template for a second liquid crystalline material that was doped with a conventional organic laser dye (pyrromethene 597). According to the information provided in the report, the lasing threshold in these structures was much higher than for other lasers discussed in this review $\left(\sim 13 \mathrm{~mJ} / \mathrm{cm}^{2}\right) .{ }^{160}$ There were also several other reports on the use of photonic crystal type structures to form conventional cavity structures, e.g. to define the mirrors of a micro-cavity ${ }^{161}$ or a 1-dimensional photonic crystal type structure $^{162}$. In many of these examples formation of the photonic crystals was achieved through selfassembly of colloids particles.

\subsubsection{Development of designs for future electrical operation}

The low threshold power densities in some of the DFB and photonic crystal feedback structures have sparked hopes that sufficient exciton densities for lasing can be achieved via electrical pumping. The electrical excitation schemes considered include both planar light-emitting organic field-effect transistor (OFET) geometries and vertical OLED architectures. For instance, rib waveguide DFB structures aligned along the channel of an OFET were reported. The position of the electrodes and the thickness of the gate dielectric were carefully optimized in these devices to achieve efficient coupling of the generated light to 
the resonant mode of the DFB structure (Figure $8 a-c) .{ }^{163}$ The emission spectrum generated under electrical operation was indeed influenced by the DFB structure but there was no evidence of lasing under electrical excitation. The lasing thresholds under optical pumping for a reference device without electrodes and for an optimized transistor geometry were shown to be very similar (4.1 versus $4.6 \mu \mathrm{J} \mathrm{cm}^{-}$ ${ }^{2}$ under $\sim 100 \mathrm{fs}$ excitation). In a different example, OFETs were based on $\sim 100 \mu \mathrm{m}$-sized single crystalline slabs of material and a diffraction grating was defined in a region of the gate dielectric located outside the active channel ${ }^{164}$. Again the grating structure influenced the emission spectrum under electrical excitation, even though there was no evidence for laser operation. Since the publication of these reports in 2009 and 2010, respectively, there have been various efforts to improve the performance of lightemitting OFETs in general, for instance by introducing multilayer structures to reduce the charge density within the emissive layer ${ }^{165}$, by introduction of a split gate structure for independent control of electron and hole injection ${ }^{166}$, by current confinement structures for increased local current density ${ }^{167}$, or by utilizing micro-cavity effects originating from the gate electrode ${ }^{168}$. It will be interesting to see how these improvements impact on the quest for electrically driven organic lasers.

OLED architectures have also been used for charge injection into DFB laser structures ${ }^{149,169}$. Using thick electron and hole transporting layers while employing a high refractive index emissive layer, one can form a waveguide in which the evanescent tails of the modes are separated from the highly absorbing metal electrodes ${ }^{170}$. In one example, the electron and hole transport layers were formed by inorganic oxides $\left(\mathrm{MoO}_{3}\right.$ and $\left.\mathrm{ZnO}\right)$ and the DFB grating was defined directly in the organic gain layer sandwiched between these via hot embossing ${ }^{169}$. Lasing was observed under optical excitation for pump fluences above $27 \mu \mathrm{J} / \mathrm{cm}^{2}$. Under electrical excitation, the gratings enhanced light extraction but the absolute efficiency was less than $0.02 \mathrm{Cd} / \mathrm{A}$, presumably due to poor charge carrier balance in these devices. In more recent work, a photo-crosslinkable green-fluorescent polymer was used in combination with UV holography and sandwiched between organic charge transport layers (hole transport layer > $500 \mathrm{~nm}$, 
electron transport layer $>175 \mathrm{~nm}$; Figure $8 \mathrm{~d})^{149}$. These structures showed improved laser performance (threshold flux of $4.8 \mu \mathrm{J} / \mathrm{cm}^{2}$ ) compared to the earlier work, where lower charge transport layer thicknesses were applied and absorption from the electrodes may have been limiting performance. Current efficiencies of $5.8 \mathrm{~cd} / \mathrm{A}$ were achieved when the devices were operated electrically, comparable to the values obtained for an optimized OLED reference device based on the same emitter polymer. 
(a)

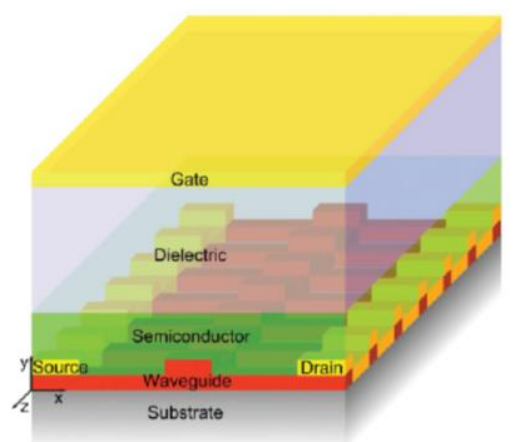

(b)

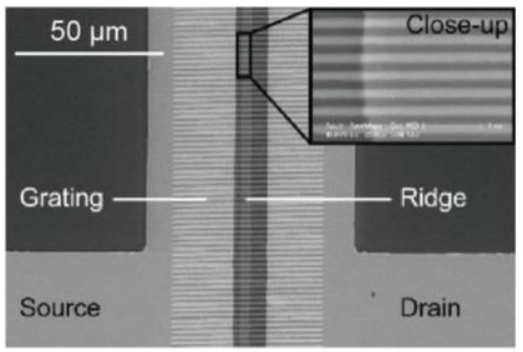

(c)
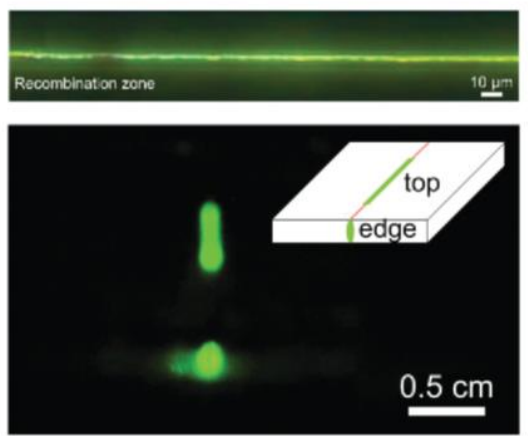

(d)

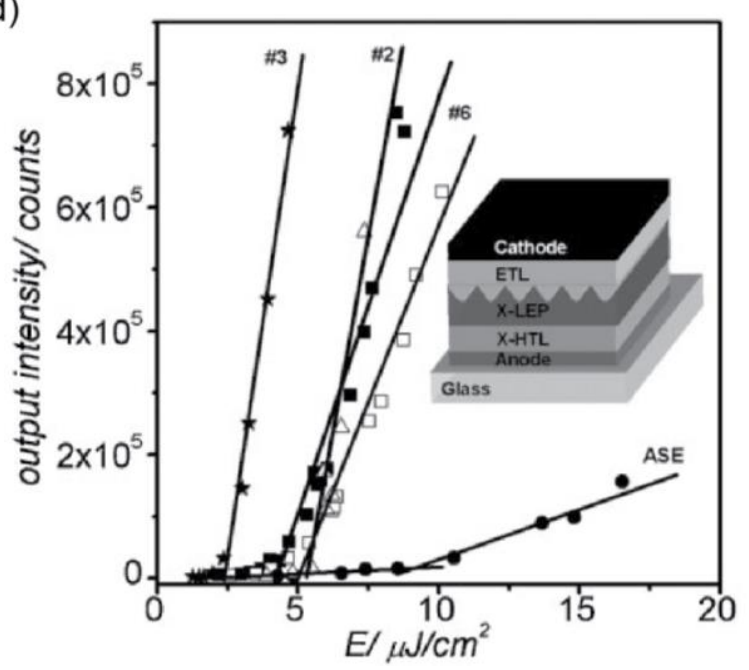

Figure 8: Examples of integration of organic DFB laser with electrical excitation schemes. (a) Schematic and (b) SEM picture of rib waveguide DFB structure aligned along the channel of an OFET. (c) Microscope pictures of top-view (above) and side view (below) electroluminescence from same OFET structure. (d) Input-output characteristics of organic DFB laser integrated into an OLED stack under optical pumping. Inset shows schematic of the laser. Reproduced with permission from [ ${ }^{149}$ ] Copyright 2010, and $\left[{ }^{163}\right]$ Copyright 2009. Wiley-VCH Verlag GmbH \& Co. KGaA. 


\subsection{Fibers and wires}

Fabry-Pérot resonators with microscopic dimensions can be prepared from fibers and wires, where the gain material is part of the fiber. In this arrangement the fiber acts as optical waveguide and the endfacets of the fiber or wire act as mirrors. Fibers with sub-micrometer diameters can be produced by electrospinning. Small fluorescent molecules such as Coumarin 334, Rhodamine 6G, and Nile Blue A Perchlorate were dissolved in a PMMA solution and then electrospun. This allowed control over fiber diameter between 100 and $600 \mathrm{~nm}$. Lasing was achieved at thresholds of $60 \mu \mathrm{J} / \mathrm{cm}^{2}$. It was argued that the observed multi-mode emission was not due to random lasing but originated from variations in fiber length, with each fiber in the sample acting as an individual Fabry-Pérot laser. ${ }^{171}$

Alternatively, electrospun fibers can be produced from conjugated polymers to obtain non-woven fleeces-i.e. networks of randomly entangled fibers-consisting entirely of gain material. Such electrospun conjugated polymer fibers were imprinted with periodic gratings using room temperature nano-imprint lithography (NIL, see section 4.3), which significantly enhanced waveguiding along the fibers. It was suggested that such a geometry can be used for fiber based DFB lasers but lasing was not demonstrated. ${ }^{172}$

To make short wires from such fibers, one can cut non-wovens using for example a microtome. However, the use of templating has been suggested as a more elegant and efficient approach. Here, porous alumina membrane templates with high surface energies were used and a conjugated polymer, e.g. polyfluorene, was infiltrated deep into the pores of the membrane by heating the polymer above its melting temperature. After cooling the infiltrated alumina template was removed and a 'forest' of nanowires was obtained. The melt processed polymer nanowires were semicrystalline and had diameters of a few hundred nanometers and lengths in excess of $1 \mu \mathrm{m}$. Evidence for lasing action presumably with the end-facets forming a Fabry-Pérot cavity - was presented, with reported lasing thresholds of around $2.8 \mathrm{~mJ} / \mathrm{cm}^{2} .173$ 
As an alternative to the template approach, compounds which easily crystallize along one axis can be used to generate nanowires. A THF solution of 2-(N,N-diethylanilin-4-yl)-4,6-bis(3,5-di-methylpyrazol-1yl)-1,3,5-triazine (DPBT), which prefers molecular packing along one crystal axis, was injected into an aqueous CTAB solution leading to surfactant-assisted formation of uniform single-crystalline nanowires. The nanowires had diameters of $<1 \mu \mathrm{m}$ and lengths of several $\mu \mathrm{m}$. The wires were pumped with femtosecond pulses to excite 2-photon fluorescence. Upon cooling to $77 \mathrm{~K}$, exciton-polariton emission occurred and lasing was observed at thresholds of $60 \mathrm{~nJ} /$ pulse. ${ }^{174}$

\subsection{Whispering gallery mode resonators}

Whispering gallery mode (WGM) resonators make use of the fact light can be trapped within a sphere or ring of gain material with higher refractive index than the environment. In a simplified picture, the whispering gallery wave can be described as multiple total-internal reflections at the curved surface of the sphere or ring. This effectively leads to circular standing waves with wavelengths that are integer multiples of the effective circumference of the structure. ${ }^{175,176}$ If the wavelength of these waves are within the gain spectrum of the used material, these waves can be amplified and lasing is obtained within the structure (Figure 3).

\subsubsection{Spherical WGM Resonators}

The $Q$-factor of WGM resonators can be very high (up to $10^{10}$ has been reported ${ }^{177}$ and is often only limited by the surface roughness of the ring or sphere. This makes the WGM approach well suited for microfluidics as liquid droplets containing a dissolved gain medium form spherical WGM resonators with extremely smooth surfaces. (A liquid-air or a liquid-liquid interface is generally atomically flat due to surface tension effects.) This approach was used in a microfluidic dropmaker, where alternating droplets of different laser dye solutions were produced. By optically exciting each droplet, a laser with alternating or switchable wavelength was obtained. ${ }^{178}$ Recently, emulsions of dye-doped microdroplets and an 
immiscible host liquid were also used as WGM lasers. This idea was combined with optical trapping which enabled fixing of the lasing droplets at a certain position and also facilitate translation throughout a sample chamber. ${ }^{179}$ In addition, droplet lasers were used to study FRET between two rhodamine laser dyes which established the dominance of non-radiative FRET over cavity-assisted radiative energy transfer. $^{180}$

In order to enable stable operation of microdroplet WGM lasers in air rather than in a host liquid, fluorescent dyes were mixed into slowly evaporating solvents. On superhydrophobic surfaces these solvents from stable microdroplets, which acted as WGM resonators. However, for Rhodamine B-doped glycerol/water microdroplets, the reported threshold fluences were extremely high $\left(750 \mathrm{~J} / \mathrm{cm}^{2}\right)$ even though the absolute pulse energies used were apparently below $40 \mu \mathrm{J} /$ pulse. In other work, a similar WGM lasers were realized that were based entirely on biomaterials that are listed by the FDA as GRAS ('Generally-Recognized-As-Safe') materials. Gain was provided by a flavin mononucleotide, a biomolecule produced from vitamin B2. Flavin containing microdroplets of glycerol with diameters of 10 to $40 \mu \mathrm{m}$ were formed by spraying onto pre-patterned super-hydrophobic poly-L-lactic acid films. Lasing was observed at $15 \mathrm{~nJ} /$ pulse, corresponding to about $1 \mathrm{~mJ} / \mathrm{cm}^{2}{ }^{88}$ More recently, glycerol-water droplets containing a fluorescent protein or a suspension of E.coli bacteria producing a fluorescent protein were used as WGM lasers. Although threshold fluences remained high $\left(46 \mathrm{~mJ} / \mathrm{cm}^{2}\right)$, the concentration of fluorescent protein required for lasing was very low $(49 \mu \mathrm{M})$. In situations where the gain material is produced by cells in situ and lasers are used predominantly for bio-sensing, the ability to achieve lasing at low concentrations of organic gain material may be more important than low threshold fluences.

As an alternative to superhydrophobic surfaces, airborne glycerol-water droplets were used and these were kept in position using optical trapping. ${ }^{181}$

Spherical WGM resonators can also be fabricated from solid gain materials, e.g. if dye doped polymer microspheres are used. For instance, WGM lasers based on Nile-Red doped polystyrene nanoparticles 
were used to follow adsorption kinetics of bovine serum albumin. When operated above threshold, the signal-to-noise ratio was improved eightfold relative to conventional sub-threshold fluorescence. In addition, the spectral linewidth of emission narrowed above the lasing threshold, thus rendering WGM lasers interesting for sensing applications. ${ }^{182}$ (Spectral shifts of the modes of WGM resonators are already widely used in bio-sensing already. However, the resonator is normally not emissive so that the mode structure needs to be probed using light from external light source, e.g. a spectrally tunable laser that is coupled to the resonator.) In further work, the dimensions of polymer WGM microsphere lasers were optimized to increase their sensitivity in the lasing regime. ${ }^{183}$

Very recently, it was shown that spherical WGM resonators can be introduced into live cells to achieve intracellular lasing (Figure 9). This demonstration was based on dye-doped polystyrene microspheres with a refractive index of 1.6 . The average refractive index inside cells is typically $1.37-1.4$ and the resulting index contrast was sufficient to achieve efficient trapping of light within the microsphere and thus obtain large $Q$-factors even for spheres with diameters well below $15 \mu \mathrm{m}$. A number of different cells types were found to be capable of internalizing the polystyrene microspheres. Thresholds for lasing were typically around $1 \mathrm{~nJ} /$ pulse, well below the onset of cellular damage. It was demonstrated that the strong dependence of the lasing spectrum on the microsphere size can be exploited for cell tagging. Once a given cell internalized a laser, the laser spectrum of this cell provided a unique optical barcode that allowed re-identification of this cell amongst a large number of other tagged cells. ${ }^{184}$ Related work looked at lasing from cells without solid WGM resonators. In particular adipocytes, which are fat cells that naturally contain a large lipid vesicle (typically diameter 40 to $50 \mu \mathrm{m}$ ), were investigated. When the adipocyte vesicle was stained with a lipophilic fluorescent dye, lasing from these cells was achieved without the need to introduce an artificial resonator structure. ${ }^{185}$ 
(a)

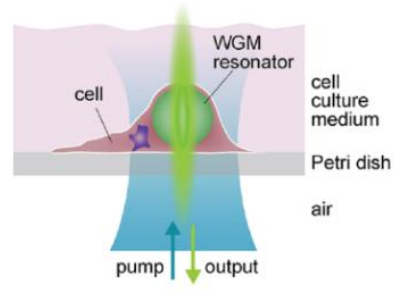

(b)

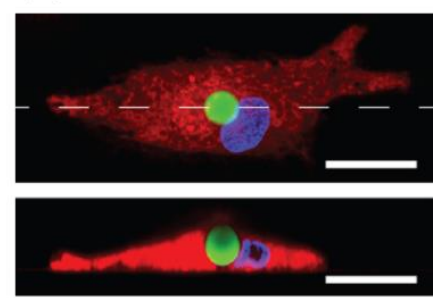

(c)

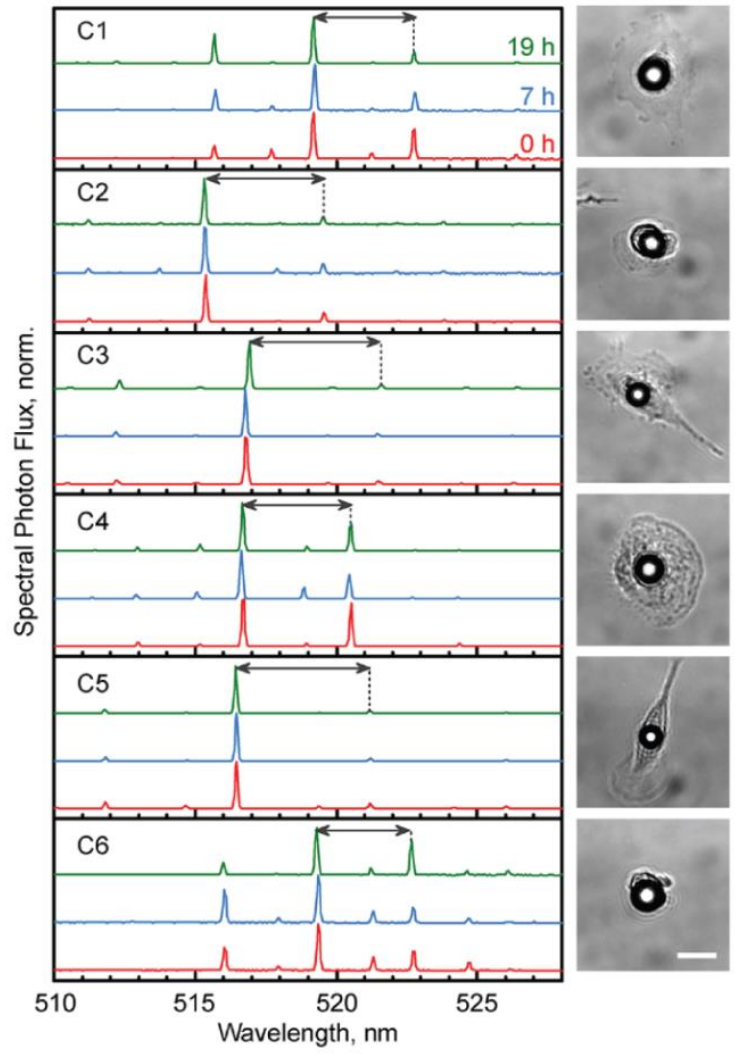

(d)

(e)

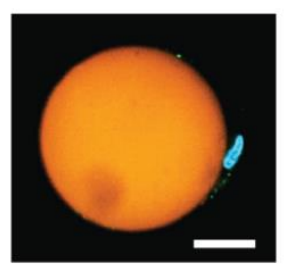

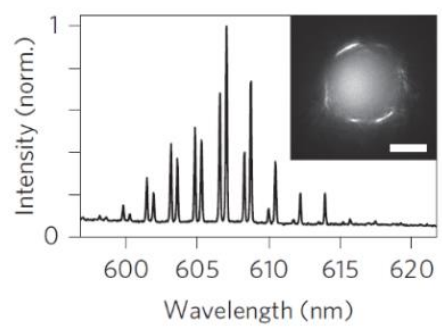

Figure 9: Whispering gallery mode resonators allow lasing within individual living cells. (a) Schematic of cell with WGM resonator and path for optical pumping and detection. ${ }^{184}$ (b) Confocal microscopy images showing a green fluorescing polystyrene bead internalized by a macrophage cell. Cell cytoplasm stained in red, cell nucleus in blue. Maximum intensity projection (top) and cross section along dashed line (bottom). Scale bar, $20 \mu \mathrm{m} .{ }^{184}$ (c) Laser spectra from six representative cells, recorded over the cause of $19 \mathrm{~h}$, illustrate that each cell has a unique laser spectrum (left). Bright-field microscopy images of cells (right). ${ }^{185}$ (d) Confocal image of an adipocyte containing a fluorescently stained lipid droplet. Scale bar, $20 \mu \mathrm{m}$. (e) Emission spectrum of an adipocyte above lasing threshold. Inset shows fluorescence image of the lasing cell. ${ }^{185}$ Reproduced from [ ${ }^{184}$ ]. Copyright 2015 American Chemical Society. Reprinted by permission from Macmillan Publishers Ltd: Nature Photonics [ ${ }^{185}$ ], copyright 2015. 


\subsubsection{Microgoblet and microdisk WGM resonators}

Microgoblets and microdisks are 2-dimensional WGM resonators. In these structures the plane in which the light circulates is fixed by the geometry of the device. Using photolithography, wet-etching and thermal reflow, PMMA microgoblets doped with the BODIPY dye PM597 were prepared and incorporated into a microfluidic device. The microgoblets formed a WGM resonator and showed lasing thresholds below $0.6 \mathrm{~nJ} /$ pulse, which allowed efficient pumping via free-space optics and a compact lowcost green laser diode. It was demonstrated that the integrated device can be applied as a refractive index sensor with a sensitivity of $10.56 \mathrm{~nm}$ per refractive index unit. ${ }^{186}$ Similar devices were realized

using Rhodamine 6G doped in PMMA. ${ }^{187,188}$ Microgoblet lasers were also produced by coating a nonemissive resonator with small molecule materials (such as a $\mathrm{Alq}_{3}: \mathrm{DCM}$ blend) using thermal evaporation. Low lasing thresholds (in this case $1.1 \mathrm{~nJ} /$ pulse) and optical pumping by laser diodes were again achieved. Applications of these devices for single molecule detection were suggested but not demonstrated yet. ${ }^{189,190}$

Microdisk resonators are similar to microgoblets, only that the microdisk is flat. Microdisks can reside on pedestals, sit flat on a low-refractive index substrate or be embedded in a low-index mold. Small molecule gain materials were thermally evaporated onto previously etched microdisk and microring molds prepared by electron-beam lithography (see 3.4.3 for more on microring structures). The resulting organic WGM lasers exhibited thresholds of around $200 \mu \mathrm{J} / \mathrm{cm}^{2} .{ }^{191}$ A microdisk prepared from silica sitting on a pedestal of silicon was coated with two layers of different low molecular weight organic dyes. First terrylene in a matrix of PMMA was spin-coated on top of the silica microdisk. Subsequently, a blend of $\mathrm{AlQ}_{3}$ :DCJTB ((4-(dicyanomethylene)-2-t-butyl-6-(1,1,7,7-tetramethyljulolidyl-9-enyl)- 4H-pyran) was evaporated onto the resonator. When pumped with a UV source, efficient energy transfer from Alq ${ }_{3}$ to DCJTB and to terrylene occurred and lasing was observed above a pump fluence of $4.5 \mu \mathrm{J} / \mathrm{cm}^{2}$. ${ }^{192}$ 
By defining two WGM microdisk lasers in very close proximity $(\sim 150 \mathrm{~nm})$, strong optical coupling between the resonators was introduced. This 'photonic molecule super-mode laser' was investigated by direct imaging of its rich mode structure, which showed the presence of modes localized across both resonators. It was suggested that this configuration may be suitable for sensing applications, in particular in aqueous environments. ${ }^{193}$

Single crystals of low molecule weight organic gain molecules, which crystallize into hexagonal disks, can also be used as WGM resonators (see sections 2.1 and 3.5).

\subsubsection{Microring-type WGM resonators}

Another widely applied optofluidic WGM resonator geometry is a ring resonator formed by an ultra-thin glass capillary that is filled with a liquid that contains the gain medium ${ }^{194}$. This approach has been used with cyanine functionalized DNA as gain medium. The DNA was programmed to assemble into tetrahedral nanostructures which allowed tuning of energy transfer phenomena between cyanine dye FRET pairs (cyanine-5, cyanine-7). In these devices the lasing thresholds were $450 \mu \mathrm{J} / \mathrm{cm}^{2}{ }^{83}$ The same resonator design was also used to analyze FRET between peptide-linked fluorescent proteins and it was demonstrated that FRET induced spectral changes were more pronounced in the lasing regime. ${ }^{83}$ The high Q-factor of WGM resonators also enabled lasing from ring resonators coated with a monolayer of fluorescent protein molecules. ${ }^{84}$

A solid-state version of the microring type resonator was reported in the form of a dye doped polymer fiber. Using a direct drawing approach, a PMMA:epoxy mixture doped with Rhodamine 6G was pulled into fibers of different diameters and lengths. Both multi- and single mode operation were demonstrated; the lowest lasing threshold reported was $53 \mu \mathrm{J} / \mathrm{cm}^{2}$. A proof of concept demonstration of refractive index sensing with a sensitivity of up to about $300 \mathrm{~nm} / \mathrm{RIU}$ was shown. ${ }^{195}$ 


\subsubsection{Further considerations on WGM lasers}

Fabrication of microdisks and microrings can involve multiple lithographic processing steps. However, recently several much simpler methods have been reported. One approach made use of the so called coffee-stain or coffee-ring effect. (This describes the fact that any dissolved or dispersed compounds accumulate at the edge of a droplet during evaporation of the solvent. This is a result of pinning of the liquid contact line at the surface and evaporation induced hydrodynamic flow.) In this way, drop casting and subsequent evaporation of an aqueous solution of a biologically produced fluorescent protein led to the formation of ring shaped deposits and thus yielded WGM ring resonators without the need for lithographic patterning and etching. ${ }^{81}$ Alternatively, dye-doped hemispherical microresonators were produced on a DBR mirror by exploiting spontaneous droplet formation on a hydrophobic surface. These resonators had lasing thresholds around $2.6 \mu \mathrm{J} /$ pulse. ${ }^{196}$ Another simple method for WGM resonator fabrication relies on ink-jet printing. Here, a triazine based polymer was printed and then coated with a layer of a different polymer material. Exposure to solvent, which only dissolved the first polymer and not the second layer, produced microdisk lasers, which resided on a thick pedestal. ${ }^{197}$

Unlike most other laser resonators, WGM lasers do not emit light in a directed fashion but instead radiate circularly away from the resonator. (In some cases this results in vector vortex beams that carry discrete orbital angular momentum. ${ }^{198}$ ) However, it was shown that by deliberately introducing specific defects into circular resonators, the emission profile of micro-disk WGM lasers can be tuned such that most light is emitted along one direction. ${ }^{199}$

Like for other resonator geometries, wavelength tuning is of great interest for organic WGM lasers. In one report this was achieved by embedding dye-doped liquid crystal droplets in a polymer matrix and applying an external electric field. The nematic liquid crystal microresonators had a tuning range of about $20 \mathrm{~nm}$ and high Q-factors up to 12,000, meaning that the tunability was approximately two orders of magnitude larger than usually achieved in solid-state microresonators. ${ }^{200}$ 
In a related approach, four liquid crystal channels were incorporated around the core of an optical fiber carrying a gain material. The core was cladded with a cylindrical photonic band gap layer. Each liquid crystal channel was flanked with individually addressable electrodes. Precise control over the omnidirectional intensity distribution from the surface of the optical fiber was achieved, which leads to intriguing opportunities, such as use as a directionally emitting laser catheter for angularly selective irradiation of pathological tissue in blood vessels. ${ }^{201}$

\subsection{Organic crystal based cavities}

Crystalline organic materials become increasingly popular for forming optical resonators as reflection at the crystal surfaces can provide substantial optical feedback and crystals offer high optical quality. One way of forming optical feedback structures from organic crystals is to make use of Fresnel reflection at their surface. Crystals can have group refractive indices of up to $~ 3.5$ and allow cavity lengths on the $100 \mu \mathrm{m}$ scale. Although the resulting Fresnel reflection at a crystal-air interface is only $~ 30 \%$, $Q$-factors of in excess of 1,000 have been reported for single-crystal Fabry-Pérot cavities ${ }^{28,202}$. This is a result of the much longer cavity lengths compared to conventional micro-cavities with length of a few multiples of $\lambda / 2$ (often sub- $\mu \mathrm{m}$ ). More recently, similar crystal cavities have also been arranged into micro-laser arrays. ${ }^{203}$

In addition to the Fabry-Pérot resonator geometry, square or hexagonal shapes are of great interest as they can provide total internal reflection and thus much better confinement of light, similar to the WGM resonators discussed in the previous section. Several examples of these configurations with different small-molecule materials were reported over recent years. ${ }^{22,204,205}$ In addition, processes were developed to arrange these crystalline organic laser cavities into defined arrays, thus providing means for implementing multiple lasers with defined properties on a single substrate. ${ }^{206}$ Very recently, WGM lasing from crystalline perovskite nano-platelets and Fabry-Pérot lasing from perovskite nanowires were also reported. ${ }^{102,103}$ 
In particular when working with single crystals, special care has to be taken when interpreting results. The dispersion relation in the crystal can lead to spectrally narrow edge emission but this must not be mistaken for lasing per-se (see section 1.4). As a whole, over recent years the community has become much better at describing observations carefully. Narrow band "conspicuous light-confined edge emission" from a single crystal embedded into and OFET for instance may be useful for certain applications but it is good to see that such observations are not hastily attributed to lasing. ${ }^{207}$

\subsection{Optofluidic resonator structures}

Achieving CW operation with organic solid-state lasers remains highly challenging. In this context, optofluidic device configurations can retain many benefits of modern organic solid state lasers (small size, simple fabrication) but at the same time can allow CW operation in a similar manner like the bulky dye jet lasers of the 1970 s and 80 s. This is because in these microfluidic optical devices the gain material is continuously replenished (either through active circulation or through diffusion), thus reducing accumulation of triplet states and photo-bleaching of the gain material in the active region of the laser. Optofluidic lasers have been reported for a range of different device geometries. A recent example based on a few $\mu \mathrm{m}$-long cavity that is formed by a planar and a spherical DBR mirror supports CW lasing for several minutes and allows tuning of the emission wavelength by translating one of the mirrors with a piezo drive (Figure 10a) ${ }^{208}$.

Even if CW operation is not achieved, optofluidic dye lasers offer interesting features, such as the ability to quickly exchange the optical gain material and hence the wavelength of the laser. Using digital microfluidics-a concept that enables controlled transport of droplets between parallel electrodes-a droplet micro-cavity dye-laser was realized that allows fast switching of the emission wavelength by electrically driven exchange of the gain medium (Figure 10b). ${ }^{209}$ Another type of optofluidic Fabry-Pérot 
micro-cavity laser was recently reported to improve the sensitivity of Enzyme-linked immunosorbent assays (ELISAs) ${ }^{210}$.

For DFB type organic laser structures, optofluidic designs are made somewhat challenging by the fact that a liquid gain material often has a lower refractive index than the surrounding solid structure containing the DFB grating. This can be addressed by using low refractive index materials such as PDMS to define the DFB structure and a high refractive index solvent for the organic gain material (like $\mathrm{DMSO}^{211}$. It has been argued that the requirement for a high refractive index core layer can be relaxed by using higher order DFB gratings and threshold fluxes of $700 \mu \mathrm{J} / \mathrm{cm}^{2}$ have been demonstrated with third order gratings. ${ }^{212}$ As an alternative, structures with a solid-state waveguide core covered with a liquid cladding containing the gain material can be used. In this configuration, amplification is restricted to the evanescent tail of the guided mode. This reduces the mode overlap and thus requires higher material gain, potentially increasing the lasing threshold. Despite this additional complication, evanescently amplified DFB lasers have been demonstrated by defining a DFB grating in a thin, high refractive-index photoresist layer and covering this layer by a liquid gain layer formed by a dye solution in DMSO and water (refractive index, 1.401, Fig. 10c-d). ${ }^{213}$ Lasing thresholds for this configuration were $9.5 \mu \mathrm{J} /$ pulse. Carefully designed 2D photonic crystal structures have also enabled optofluidic lasing from methanol based solutions of Rhodamine $6 \mathrm{G} .{ }^{157}$ Very recently, evanescent amplification has also been achieved with a purely water-based DFB optofluidic laser which may be important to enable a range of bio-sensing applications. ${ }^{214}$

A very large proportion of the literature on whispering gallery mode resonators uses optofluidic configurations, in part because microdroplets and capillaries form very high quality WGM resonators. These devices were therefore discussed in section 3.4 together with other WGM designs. 
(a)

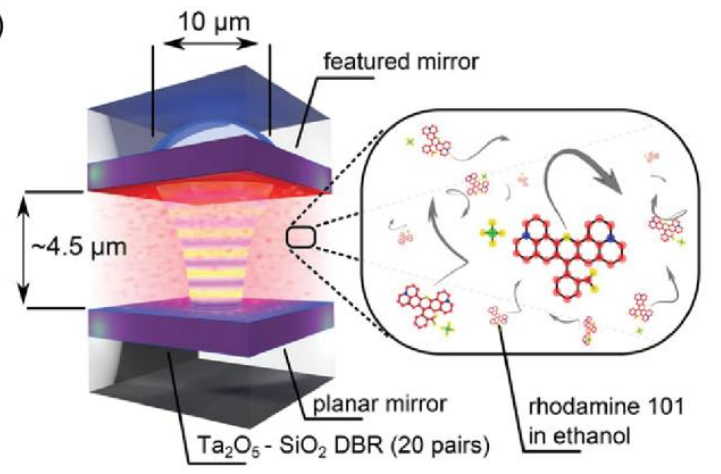

(b)

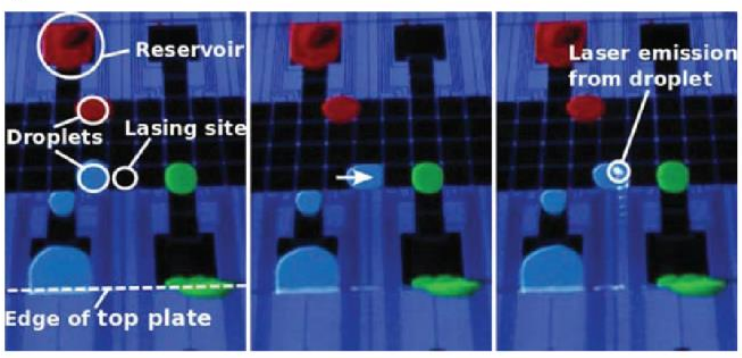

(c)
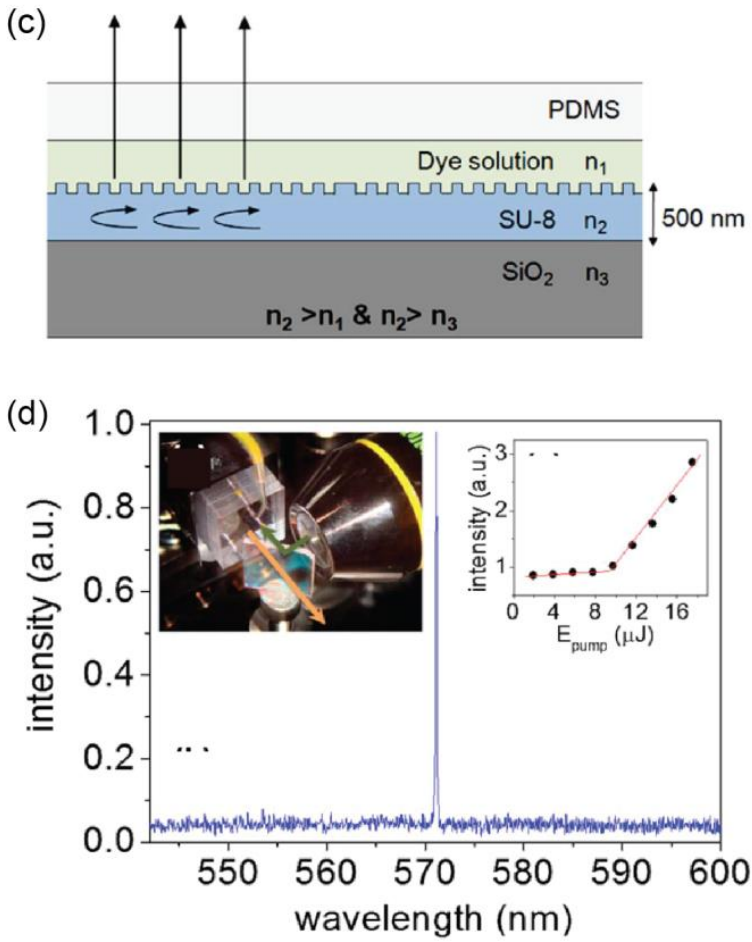

Figure 10: Different designs for optofluidic organic lasers. (a) Schematic of an optofluidic micro-cavity laser. By exploiting diffusion of luminophores within a droplet of solvent, continuous wave operation can be achieved in small optofluidic lasers. (b) Photographs of a laser that uses digital microfluidics for controlled delivery of droplets containing laser dyes emitting light of different wavelengths to a planar micro-cavity. (c) Schematic and (d) laser spectrum of a circular DFB grating based optofluidic laser. Insets in (d) show picture of the device with illustration of the excitation/detection geometry (left) and the input-output characteristics of the laser (right). Reproduced in part from $\left[{ }^{209}\right]$ with permission of The Royal Society of Chemistry. Reproduced from $\left[{ }^{208}\right.$ ] with permission. Copyright 2015 Wiley-VCH Verlag GmbH \& Co. KGaA. Reprinted from [ $\left.{ }^{213}\right]$ with the permission of AIP Publishing. 


\subsection{Random lasers}

Laser emission can also be observed in the apparent absence of a resonator structure. For example lasing was observed in plain spin coated conjugated polymer films deposited on flat substrates. ${ }^{215,216}$ Here, the lasing was attributed to multiple scattering at refractive index inhomogeneities (e.g. morphology variations, impurities, crystallites) that provided a closed path for light and thus optical feedback. For the same reason, colloidal glasses, which are amorphous and non-ordered systems by their very definition, also showed lasing when backfilled with a gain medium or when the colloids themselves carried laser dyes. ${ }^{217}$ Random lasers often exhibit a multitude of narrow laser lines, which change position and intensity upon variation of the excitation density, area and position. ${ }^{218}$ Resonance in random lasers is generally produced by multiple scattering following a random-walk and the formation of localized states in the ill-defined and disordered gain medium. ${ }^{219,220}$

A possible application for random lasers is in sensing of small molecules, e.g. explosives. Monodisperse titania or silica nanoparticles were embedded in a polyphenylene derivative, resulting in random lasers with a threshold of $191 \mathrm{~mJ} / \mathrm{cm}^{2}$ (for titania) and $3 \mathrm{~mJ} / \mathrm{cm}^{2}$ (for silica). ${ }^{71,221}$ Upon exposure to TNT, the optical gain of the used laser material was significantly lowered, which prevented lasing and thus allowed for detection of traces of TNT in the ppb range. ${ }^{221}$ (The idea of TNT detection has also be pursued with other resonator geometries, in particular with DFB gratings; see section 7.2.) Another exciting application of random lasers is for speckle free laser based imaging. In an initial demonstration of this concept, the low spatial coherence laser light provided by Rhodamine molecules in a dispersion of polystyrene spheres was exploited to achieve low-speckle full-field/wide field imaging through a scattering medium. ${ }^{222}$

To combine the advantages and features of random lasers with those of periodic lasers, quasi-periodic Fibonacci gratings were produced in an inorganic gain material and for these gratings Bragg peaks were observed together with random laser peaks. ${ }^{223}$ Such a system therefore makes use of the advantages of 
both lasing mechanisms, allowing control over strength and angle of the surface emission. Quasicrystalline lasers could also be used for intra-cavity nonlinear mixing and may in the future be implemented in compact multi-color lasers with high spectral purity of the emission lines. However, such quasi-periodic structures have not yet been realized in organic materials.

\subsection{Plasmonic structures}

Plasmon resonances represent another method of confining light. Compared to dielectric waveguides and feedback structures, the volume to which they confine light (mode volume) is often much smaller and this can result in a local enhancement of the electric field. Plasmonic field enhancement is widely used in sensing applications, e.g. for surface plasmon resonance sensors or surface-enhanced Raman spectroscopy. There is also interest in using the plasmon-mediated concentration of the electric field in the vicinity of metal surfaces or metallic nanostructures to design better lasers. In the following we will review some of the developments in this field. References $[11,224,225]$ provide more detailed reviews on small lasers and optical amplifiers that use metallic or plasmonic structures in combination with organic materials.

Local field enhancement is often considered beneficial for designing more compact or potentially even more efficient lasers. However, surface plasmon modes generally also show much larger losses than dielectric modes, i.e. the modal absorption coefficient $\alpha$ that was introduced in section 1.2 can be drastically increased. As any loss has to be compensated by optical gain for lasing or amplification to occur, the development of plasmonic lasers based on organic gain materials has been challenging so far.

One strategy towards organic lasers and amplifiers based on plasmon resonances is to make use of so called long-range surface plasmon polariton (LRSPP) modes. These modes propagate on both sides of thin metal films (thickness usually $<30 \mathrm{~nm}$ ) and show much lower losses than conventional surface plasmon modes, which propagate on a bulk metal surface. Waveguides for LRSPP modes have recently 
been combined with organic gain media to compensate the residual propagation loss present in these. Using this approach, net amplification, i.e. complete compensation of all losses plus additional gain, has been demonstrated for gold LRSPP waveguides. These waveguides were either embedded in a polymer blend based on a PPV based gain material ${ }^{226}$ or combined with an optofluidic device that passed a solution of an IR emitting organic dye (IR140) across the waveguide ${ }^{227}$. In both cases the ability to adjust the refractive index of the gain medium turned out to be important to control the losses of the LRSPP mode. More recent work demonstrated LRSPP waveguide amplifiers based on small molecule materials and showed the integration with grating-based input and output couplers. This work also reported detailed analysis of the achievable gain at different pump powers and for different samples. ${ }^{228}$ The propagation loss of LRSPP waveguides critically depends on the smoothness of the metal film. ${ }^{229}$ Tuning of the surface energy between the metal and underlying substrate material enabled fabrication of extremely smooth gold and silver films and enabled fabrication of continuous metal films that were thinner than the traditionally assumed percolation limit for these materials (approximately 10 to $15 \mathrm{~nm}) .{ }^{230,231}$ Despite these advances, to the best of our knowledge, no organic LRSPP laser has been demonstrated so far. However, designs and models for such a device - with DBR or DFB gratings embedded in the waveguide - have been reported. ${ }^{232}$

Recently, loss compensation has also been attempted for silver nanowires deposited on Rhodamine doped PMMA layers but only partial compensation of the losses was achieved. ${ }^{233}$

An alternative to propagating surface plasmons is the use of localized non-propagating modes, which are supported for instance by metallic nanoparticles. Stimulated emission of surface plasmons is expected in these structures if sufficient optical gain is provided by the medium surrounding the nanoparticles. Such a device is often referred to as spaser (surface plasmon amplification and stimulated emission of radiation) although this term has also been used for other plasmon-based lasers. Due to the large losses induced by metallic particles, the $Q$-factors expected for these localized plasmon modes are generally 
well below 100, which means that the required material gain is much higher than in other optical feedback structures. To our knowledge, there have been only been very few demonstrations of true spasers. These devices were based either on dye-coated gold nanoparticles ${ }^{234}$ or silver nanorods embedded in a dye-loaded polymer matrix ${ }^{235}$. In both cases, the experiments involved a large ensemble of particles ( $>10^{6}$ particles) and demonstration of spaser action from a single isolated particle has yet to be shown. In related work based on a similar sample structure, strong coupling of the gain material to the plasmon mode was demonstrated by a reduction in excited state lifetime but here no spaser action was claimed. ${ }^{236}$

A device configuration that makes use of localized plasmon modes but relaxes the gain requirements are ordered or random arrays of plasmonic nanocavities and there are now a number of reports on organic lasers based on such arrarys. ${ }^{235,237,238}$ By using a periodic subwavelength hole array perforated in a metal film, directional emission was recently achieved with the plasmonic array approach. ${ }^{239}$

\subsection{Conclusion}

A large variety of resonators are compatible with organic gain materials and this has allowed the development of a large number of different organic lasers with different characteristics and advantages. Some geometries, such as DFB gratings and WGM resonators, offer direct access to the organic gain materials and are therefore of interest for applications in sensing and detection (see section 7.2 for outlook on applications of organic lasers). Other resonators are particularly attractive due to their high Q-factors or facile preparation routes (see section 4 for fabrication strategies). Plasmonic structures can supply feedback on scales much smaller than the typical resonator geometries, with footprints much smaller than the wavelength of visible light. Therefore, it is unlikely that an ideal resonator design for organic lasers will emerge. Instead, the different geometries allow researchers to select the most suitable design for each application or scenario. 


\section{Fabrication}

One of the main advantages of organic lasers is that they can be produced relatively quickly and at low cost and that their exact structure and hence their optical characteristics can be easily adjusted. However, organic semiconductors are generally not compatible with conventional lithography and etching processes, which are applied to define photonic micro- and nanostructures in inorganic semiconductors. The success of organic laser technology is therefore closely linked to advancing the relevant process technology. This is particularly relevant for distributed optical feedback and photonic crystal structures, which require definition of wavelength-scale patterns within or close to the organic gain material. Traditionally, the laser resonator for these structures has been formed by spin-coating or evaporating a gain material onto an inorganic, dielectric substrate, into which a DFB resonator structure has previously been imposed. These DFB structures can be produced by electron-beam lithography followed by subsequent dry etching and some structures with certain fixed grating periods are commercially available. While this is convenient for material development and proof-of-principle demonstrations of lasing, more efficient and flexible fabrication methods are clearly desirable and a number of alternative distributed feedback resonator designs and associated fabrication methods have therefore been developed.

\subsection{Topical direct writing techniques}

Electron beam lithography is a means to precisely define 2-dimensional nanostructures. Electron beam lithography, also termed "e-beam writing", is a direct writing technique, in which each feature is drawn into a thin layer of an electron-sensitive photoresist with a focused electron-beam that is steered across the substrate using the same type of electron optics used in scanning electron microscopes. In section 2.3 we already discussed a crosslinkable VE-PFO polymer, into which DFB laser gratings can be patterned using e-beam lithography at low acceleration voltages and currents, i.e. using relatively mild electron 
exposure (Figure 11). ${ }^{60}$ At higher currents and acceleration voltages, the used electron beam may oxidize the conjugated polymer, which would result in a loss of fluorescence. While this is generally not desirable, it was shown that this effect can also been utilized to pattern a ethylhexyl functionalized polyfluorene; however, applications in laser geometries were not yet presented for this technique. ${ }^{240}$

(a)

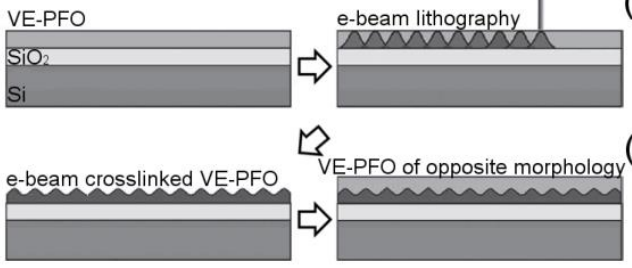

(b)

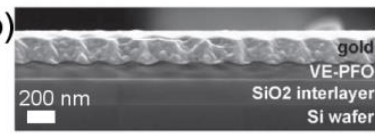

(c)

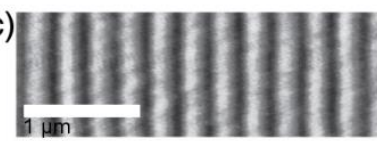

(d)

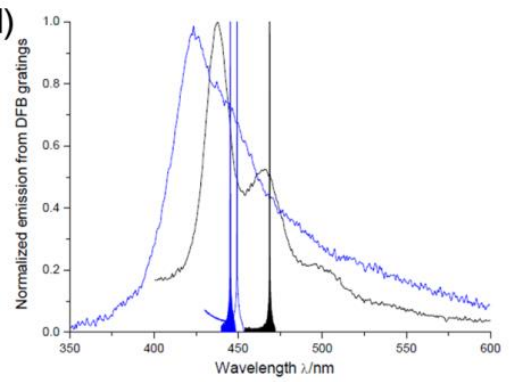

Figure 11. Direct e-beam writing. (a) Schematic of a process which directly patterns an organic gain material: A film of VE-PFO is exposed to an e-beam to induce crosslinking of the conjugated polymer. Subsequent development step removes non-exposed polymer revealing the grating. An additional layer can be deposited as the crosslinked VE-PFO is not attacked by common solvents. ${ }^{60}$ (b) SEM image of a cross-section of the grating and (c) AFM image of topography from top. ${ }^{60}$ (d) Fluorescence and lasing spectra of the two e-beam written VE-PFO based DFB gratings. Reproduced from $\left[{ }^{60}\right.$ ] with permission. Copyright 2011 Wiley-VCH Verlag GmbH \& Co. KGaA.

Even though e-beam lithography represents one of the most precise, exact and versatile methods for nanopatterning available today, it requires long processing times, high vacuum and sophisticated control electronics, which makes it expensive. Several other direct writing techniques have been explored recently. By changing from an electron beam to a laser beam, the problem of vacuum processing can be circumvented and the optics for focusing and steering the laser are generally easier to implement. However, the minimum feature size achievable with direct laser writing is much worse than for e-beam lithography. Since typical laser resonators require feature sizes of around $200 \mathrm{~nm}$, laser writing techniques, which overcome the conventional free-space diffraction limit, have been considered to directly inscribe DFB gratings into organic gain media. One such tool is scanning near-field optical lithography (SNOL). In SNOL a focused laser beam is passed through a sub-wavelength aperture (e.g. a $50 \mathrm{~nm}$ hole) and a substrate with a photosensitive gain-medium is placed in very close proximity of the aperture. Using this approach oxetane functionalized fluorene polymers mixed with a PAG were photo- 
crosslinked and feature sizes of $60 \mathrm{~nm}$ (FWHM) were achieved (film thickness after removing noncrosslinked materials with solvent was $160 \mathrm{~nm}){ }^{241}$ In related work, a soluble poly $(p$-xylene tetrahydrothiophenium chloride) was exposed to SNOL. The focused laser induced an elimination reaction, producing a less-soluble but high optical gain version of the material PPV and a small molecule as a side product. The side product was removed together with unexposed polymer in a development step. Feature sizes of $55 \mathrm{~nm}$ in width (FWHM) and about $10 \mathrm{~nm}$ in height were achieved.

A different approach to overcome the conventional free-space diffraction limit is 2-photon lithography. Here, a high-power red or near infrared wavelength laser is focused tightly into the sample so that efficient 2-photon absorption occurs at the focal point (i.e. effectively the red light from the laser acts like blue light at the focal point). This can then induce a photochemical process in the material, e.g. initiate crosslinking of the material. The focal point can be scanned in three dimensions to define arbitrary 3-dimensional structures. This is possible because photochemistry is only triggered at the focal point and material remains unaltered in out-of-focus regions where the intensity is much weaker and hence virtually no 2-photon absorption occurs. Until now, gain-materials have not been structured directly into laser resonators using 2-photon processes. However, inert photoresists were patterned into a 2-dimensional egg-box grating, an $\mathrm{Alq}_{3}: \mathrm{DCM}$ layer was evaporated onto the grating and investigated regarding the laser performance. ${ }^{242}$ Other tools originally developed for surface analysis can be adapted to direct writing techniques. SNOL for example is an adaptation of scanning near field microscopy (SNOM). Atomic force microscopy (AFM) has been adapted to dip-pen lithography (DPL). In DPL, an adapted AFM tip is placed into a solvent or a solution of a gain material and then placed on a surface to draw lines with diameters as small as tens of nanometers. Using DPL, a film of amorphous PFO was exposed to toluene. In this way domains with $\beta$-phase morphology with dimensions of a few hundred nanometers were directly written into a PFO film. ${ }^{243}$ The PFO films were patterned with grating 
structures and the formation of a photonic bandgap was observed but lasing from such a device was not yet demonstrated.

\subsection{Interference lithography and holographic patterning}

Similar to the processes of direct writing and photolithography described above, laser interference can also be used to pattern a substrate. The interference fringe pattern generated by two laser beams incident on the sample under different angles leads to an intensity modulation in one dimension, which can be used to produce a 1-dimensional grating structure (Figure 12). This process is also called holographic patterning. Imposition of such gratings either directly into a conjugated polymer, e.g. by photo-crosslinking ${ }^{149}$, or into an optically transparent photoresist layer that remained as a part of the final device ${ }^{244,245}$ allowed their use as laser resonators with low thresholds. Both approaches avoided additional etching steps for transfer of the grating structure, thus simplifying device fabrication and ensuring that the grating structure is well preserved.

(a)

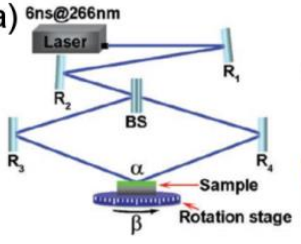

(b)

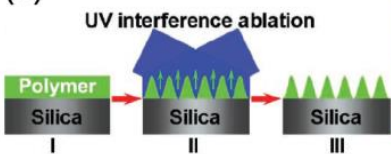

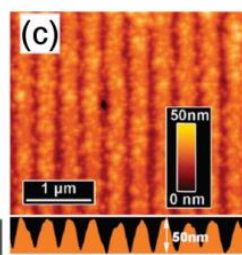

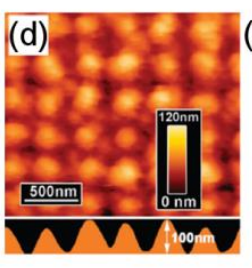

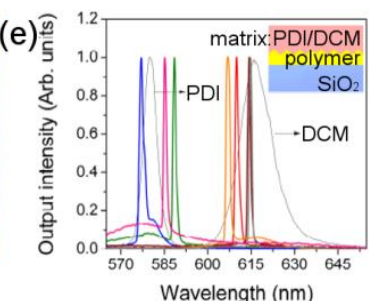

Figure 12. Interference patterning: (a) A laser beam is split into two beams, which are then recombined on a photoresist film, creating an interference pattern. ${ }^{247}$ (b) After illumination and development a corrugated surface is obtained. ${ }^{247}$ (c) AFM image of the produced grating. ${ }^{247}$ (d) If a third beam is added at an angle of $90^{\circ}$ with the other two laser beams a 2-dimensional grating is created. (e) Depending on the grating period and the gain material applied (PDI, DCM), the lasing wavelength can be tuned. ${ }^{310}$ Reproduced from [ ${ }^{247}$ ] with permission. Copyright 2011 Wiley-VCH Verlag GmbH \& Co. KGaA.

Similar to the in e-beam induced oxidation of organic materials for controlled local degradation, laserinterference lithography can induce to photo-oxidation in a conjugated polymer or even ablation of the material if sufficiently high intensity is used. This was exploited for the generation of 1-dimensional surface relief gratings in films of $\mathrm{Alq}_{3}$ :DCM or a 9:1 F8BT copolymer. These structures showed lasing with 
$\lambda_{\text {laser }}=646 \mathrm{~nm}\left(E_{\mathrm{th}}=41 \mu \mathrm{J} / \mathrm{cm}^{2}\right)$ and $569 \mathrm{~nm}\left(E_{\mathrm{th}}=130 \mu \mathrm{J} / \mathrm{cm}^{2}\right)$, respectively. ${ }^{246,247}$ A 2-dimensional (eggbox) grating was produced either by rotating the substrate by $90^{\circ}$ after the first ablation cycle, followed by a second exposure, or by using interference from three laser beams (Figure $12 \mathrm{~d}$ ). ${ }^{247}$ The resulting 2dimensional grating laser exhibited a lower threshold than the 1-dimensinonal grating, presumably due to better mode confinement $\left(E_{\mathrm{th}}=100 \mu \mathrm{J} / \mathrm{cm}^{2}\right.$ rather than $\left.130 \mu \mathrm{J} / \mathrm{cm}^{2}\right)$.

Laser interference lithography is generally faster than the direct writing methods described above as the grating is produced by a one-shot exposure rather than by line-by-line drawing. However, the increased speed comes at the cost of freedom of design as holographic patterns are usually periodic, often sinusoidal in nature.

\subsection{Nanoimprinting and micro-contact printing}

Nanoimprint lithography (NIL) relies on nano-patterned master structures - often referred to as stamps or templates - which are usually produced by conventional nanofabrication techniques, e.g. using one of the methods described above. However, this master can be reused multiple times to transfer a pattern into a gain medium of choice. This transfer is typically performed by the following steps: First, the master that contains the desired nanostructure - usually a periodic DFB grating - is coated with an elastomer-precursor. The precursor is then cured thermally or photochemically to produce a crosslinked but relatively flexible material. This process replicates the grating structure in the flexible polymer film, which can now be used repeatedly as an intermediate master or mold. In an early demonstration of this approach, the commercial photoresist SU-8 was doped with the dye Rhodamine 590 and the mixture was deposited on a nano-patterned mold. The sample was then irradiated with UV-light to cure the SU-8 and transfer the original nanostructure into the SU-8 layer. The elastomeric mold was subsequently removed simply by peeling it off yielding a device that exhibited the typical laser emission characteristics

of Rhodamine. ${ }^{248}$ Later, the process was improved by using an optically more transparent NIL resist (SU-8 
absorbs slightly in the blue part of the spectrum) and switching to Rhodamine 6G. In addition, the mold in this example consisted of a hexagonal lattice of cylinders, which resulted in a 2-dimensional photonic crystal consisting of an array of hexagonal holes in the gain medium. ${ }^{249}$ The lasing wavelength in these structures was tuned by adjusting the grating period, which was facilitated by the broad gain spectrum of the Rhodamine 6G dye.

The NIL process can be easily combined with dry and wet etching processes to pattern materials that cannot be imprinted directly. In an example of a conjugated polymer laser, a substrate was coated first with a metal oxide (flowable oxide; FOX) and then with a NIL resist. Different DFB gratings with symmetric and asymmetric lattices were transferred into the NIL resist. The substrate was then exposed to an oxidative dry etching cycle in order to transfer the pattern from the nanoimprinted layer into the FOX layer. The residual NIL resist was removed by oxygen plasma etching and BBEHP-PPV was spincoated on top of this structured oxide layer. ${ }^{142}$ This process allowed the production of gratings with extremely deep grooves of up to $150 \mathrm{~nm}$ and illustrated that - in particular in combination with established semiconductor patterning technology - NIL is capable of producing extremely high precision gratings. This was also evidenced by the low laser thresholds (in the sub $\mu \mathrm{J} / \mathrm{cm}^{2}$ regime) achieved with this process, which have allowed pumping with commercially available inorganic UV-LEDs. ${ }^{137,138}$

Free standing 2-dimensional grating resonators can be produced by combining conventional photolithography with NIL. In one example of this, the protruding features of a transparent flexible imprint mold were coated with a thin metal layer. The mold is then imprinted into a dye-doped transparent photoresist and exposed to UV light through the mold, which cured the resist everywhere but in the regions shadowed by the thin metal film. After removal of the mold and development of the resist, free standing 2-dimensional photonic crystal band-edge lasers were obtained. ${ }^{250}$ In other work, the nanoimprint stamp was embossed into a gain medium that was heated to above its glass transition temperature to render the material soft. After cooling and delamination of the stamp, the desired 
structure was transferred. Lasing was demonstrated for Ormocore films doped with Pyrromethene 597 and for polystyrene films doped with IND-PBI. ${ }^{251,252}$

The flexible molds used in the different versions of NIL can also be used for micro-contact printing. Micro-contact printing works by creating conditions where the transferred material adheres less well to the mold than to the target substrate. To achieve this, the mold may be surface-functionalized with fluorine-groups or be composed entirely of a fluorinated material. In an early example, a 1-dimensional master-grating was first transferred into a polymer film which was coated with a $1 \%$ Teflon solution. This mold was then coated with a $35 \mathrm{~nm}$ layer of $\mathrm{Alq}_{3}$ and a subsequent $15 \mathrm{~nm}$ layer of NPB (4,4'-bis[N-(1naphthyl)- $N$-phenylamino]biphenyl) by thermal evaporation in high vacuum. Finally, the mold was pressed onto a glass substrate which already contained an unstructured $200 \mathrm{~nm}$-thick layer of Alq3:DCM applying (pressure, $5 \mathrm{MPa}$; temperature, $55^{\circ} \mathrm{C}$ ). During this process, the Alq3/NPB bi-layer was transferred from the grating mold to the substrate, but only at the protruding features of the mold (Fig. 13a,b). The resulting grating of 1-dimensional lines of Alq3/NBP on a layer of Alq3:DCM exhibited single mode laser emission with thresholds on the order of $1 \mathrm{~mJ} / \mathrm{cm}^{2} .{ }^{253}$ In other work, quantum dots were transferred onto films of organic gain media to form a grating structure. The laser performance of these structures was compared to polymer-only lasers based on the same conjugated polymer but structured via holographic interference lithography. The quantum dot devices exhibited lower lasing thresholds than the all conjugated polymer devices (45 versus $65 \mu \mathrm{J} / \mathrm{cm}^{2}$ ), which was attributed to the high refractive index of the quantum dot grating yielding stronger distributed feedback in these devices (Figure 13c-f). ${ }^{254,255}$ 
(a)

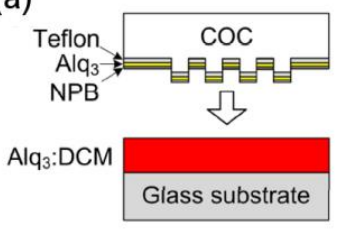

(b)

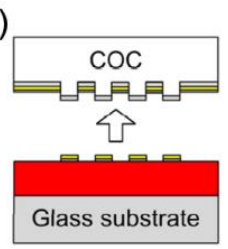

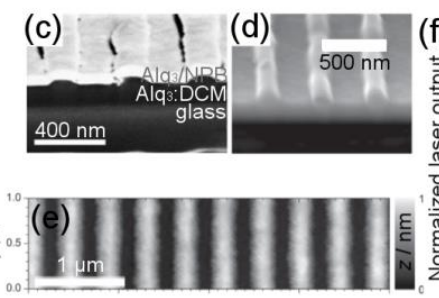

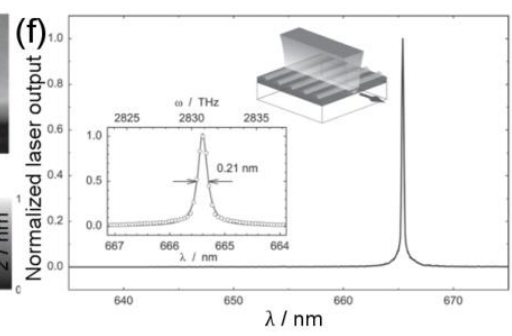

Figure 13. Micro-contact printing for fabrication of DFB gratings. (a) Schematic of the micro-contact printing process. The pattern is produced by transferring material from the protruding features of a mold to the substrate. ${ }^{253}$ (b) Transfer is facilitated by using a mold material to which the transferred material adheres weakly. ${ }^{253}$ (c) SEM image of a cross-section of a transferred $\mathrm{Alq}_{3}: \mathrm{NPB}$ pattern on an $\mathrm{Alq}_{3}: \mathrm{DCM}$ layer. ${ }^{253}$ (d) SEM image of microcontact printed quantum dots on a conjugate polymer film. ${ }^{255}$ (e) AFM image of the same quantum dot grating. ${ }^{255}$ (f) Emission spectrum of laser with micro contact printed grating of quantum dots ${ }^{255}$. Reproduced from $\left[{ }^{255}\right]$ with permission. Copyright 2014 Wiley-VCH Verlag GmbH \& Co. KGaA.

\subsection{Structure formation via colloidal self-assembly}

Sub-micrometer particles exhibit Brownian motion and when deposited from dispersion under suitable conditions, they spontaneously self-assemble into crystalline structures. The self-assembly is driven by van-der-Waals interactions between the particles and by the capillary forces arising when the dispersing medium evaporates. ${ }^{256}$ The resulting assemblies often have hexagonal symmetry with fcc or bcc crystal lattices. The defining crystal lattice distances depend on the size of the particles and can be on the order of the wavelength of visible light. This makes colloidal crystals ideal materials for the creation of 3dimensional photonic crystals. ${ }^{5}$ To utilize these materials as laser resonators, monodisperse dielectric particles are usually first assembled and then backfilled with a gain material. Optionally, the original dielectric materials can be removed to enhance the refractive index contrast. In some earlier work (before 2007), monodisperse silica particles were assembled, backfilled with laser dye solutions (of oxazine and stilbene) and pumped with picosecond excitation pulses. Lasing from the dye-solution infiltrated colloidal crystals was observed with lasing thresholds around $320 \mu \mathrm{J} / \mathrm{cm}^{2}{ }^{257}$ This approach was translated to form solid state lasers by mixing the dielectric silica colloids mixed with a laser dye solution containing a dissolved dielectric polymer. Upon deposition of this mixture, the solvent evaporated, and the colloids assembled into crystalline arrays. The polymer/laser dye mixture concentrated around the crystalline assembly until all solvent was removed. The colloidal silica crystal was then removed by 
etching in hydrofluoric acid. Hydrofluoric acid is a weak acid leaving the organic materials intact while efficiently dissolving the silica beads. The resulting inverse opal crystal exhibited laser emission at $E_{\text {th }}=$ $6 \mathrm{~mJ} / \mathrm{cm}^{2}$. However, the emission was spectrally rather broad (with FWHM of $3.5 \mathrm{~nm}$ ), which might indicate that the observed emission actually represents ASE (see characteristics of lasing in section 1.4) ${ }^{258}$ If the dielectric solid polymer matrix is replaced by a silicone elastomer, for example a transparent siloxane, the self-assembled photonic crystal can be easily deformed, which alters the photonic bandgap of the assembly. Using this approach, the stop-band in the (111) direction was shifted from deep red into the green part of the spectrum. Rhodamine dyes exhibit good solubility in silicones and it was suggested that a tunable photonic crystal loaded with Rhodamine can be used as a force or deformation sensitive tunable laser. Tunability from 588 down to $655 \mathrm{~nm}$ was demonstrated. The devices exhibited thresholds of $13.5 \mathrm{~mJ} / \mathrm{cm}^{2}$ with single line emission and linewidths of $0.06 \mathrm{~nm} .{ }^{259}$ In a similar approach, a micro-cavity laser was produced. Here, two colloidal crystals were encapsulated by a silicone matrix as described above but these crystals were then used as mirrors to sandwich a rigid polymer film doped with rhodamine dye. In this case deformation of the photonic crystal caused a spectral shift in the reflectance of the mirrors, away from the rhodamine emission. In this way it was possible to produce bendable colloidal crystal lasers with thresholds of $412 \mu \mathrm{J} / \mathrm{cm}^{2}{ }^{260}$

\subsection{Encapsulation}

During excitation of organic emitters, long lived excited states can be generated (see section 1.3). These triplet states cause issues associated with lifetime and stability of organic laser. Due to their long lifetimes, molecules in the triplet state can be very reactive and will quickly react with any oxygen present. Reducing the triplet concentration is therefore expected to slow down degradation of organic lasers and this may become particularly important for electrically pumped lasers because of additional

triplet-associated degradation pathways that also occur in the absence of oxygen (see section 6.3 ). ${ }^{261}$ 
However, the predominant pathway of degradation under ambient conditions is often photo-oxidation which can lead to formation of keto-species as is often seen in fluorene derivatives. ${ }^{261-263}$

To prevent oxidation, experiments are usually conducted in an inert atmosphere, e.g. inside a nitrogen filled glovebox or within a vacuum chamber. To use organic lasers in real world applications, devices obviously need to be encapsulated in a more compact way. For optically pumped lasers this can be achieved by simply dispersing the gain medium in a transparent oxygen impermeable matrix. ${ }^{264}$ Small molecule laser dyes, e.g. rhodamines, are frequently encapsulated in polymer matrices, such as PS and PMMA. To reduce oxygen diffusion through such polymers, trimethoxy silane groups were incorporated into the periphery of methacylate polymers and the amount of silane groups was varied. However, silane groups were found to disturb the polymer morphology, inducing a tendency for crystallization, and in addition lowered the glass transition temperature and the refractive index. Still, the polymer encapsulation increased device lifetimes of small molecule rhodamine and pyrromethane based lasers with holographically defined gratings. The encapsulated devices showed no sign of degradation after more than 100.000 pulses. ${ }^{264}$

However, this matrix encapsulation approach is not suitable for future electrically driven geometries. Here, instead the entire device needs to be encapsulated to prevent ingress of oxidizing species. Similar challenges exist for other organic semiconductor based devices, in particular for OLEDs, and there are now processes in commercial use that achieve tight encapsulation for these. One would therefore expect that this technology can be transferred to organic lasers. For completeness, we will briefly summarize some recent reports that specifically deal with encapsulation of organic lasers. Development and testing of several approaches were also described in ${ }^{4}$. Encapsulation employed spin-coatable or liquid processable sol-gel silicates $^{265}$, organic-metal oxide hybrid composites, fluorinated ${ }^{137,266}$ and water processable polymer films ${ }^{267}$ and combinations thereof. ${ }^{268}$ Micro-contact deposited nanogratings of $\mathrm{Alq}_{3}$ :DCM were encapsulated with PMMA (sold by HesaGlas ${ }^{\circledR}$ ) and COP (a cyclic olefin polymer sold by 
TOPAS $^{\circledR}$ ). The polymer layer thickness was between 250 and $500 \mu \mathrm{m}$ and full (4 inch) wafers were processed to confirm industrial feasibility of this approach. For PMMA, an 11-fold and for COP a 3-fold enhancement in lifetime over a non-encapsulated device were achieved. ${ }^{269}$ Conjugated polymers and oligomers are often deposited from organic non-polar solvents and therefore the deposition of nonpolar encapsulating polymers (such as polymethacrylates and polystyrene) can be problematic as solvent processing may destroy the underlying laser gain medium. As an alternative, water soluble encapsulating polymers were proposed (although brining the gain material in contact with water is may damage the material). Polyvinylalcohol (PVA) was applied to encapsulate $T_{3}$ truxene oligomers on a DFB grating. The PVA encapsulant increased the photostability under pumping conditions by a factor of $40 .^{267}$

\subsection{Conclusion}

A variety of fabrication techniques have been adapted to organic materials, ranging from serial beamwriting to parallel large area imprinting techniques. Some of these are borrowed from inorganic semiconductor processing, others are exclusive to soft organic materials and solvent processing procedures. High quality DFB gratings are still often best produced by beam-writing techniques. Although beam writing is inherently slow, it allows for well-defined grating periods and enables adjustment of periodicity between batches. However, if the same grating period is used repeatedly, imprinting is highly attractive as the resonator structure can be imposed much faster. If there is some flexibility with regards to the precise shape of the grating, holographic techniques are of great interest as they combine quick adjustment of grating period and high throughput. Recent results show that under optimized conditions nanoimprint and holography both offer precision akin to serial beam-writing techniques. With regard to 3-dimensional feedback structures, the resolution of direct writing techniques in z-direction remains limited and as a result 3-dimensional photonic crystals have mainly been produced using self-assembly of monodisperse particles. While self-assembly is an extremely low-cost and facile way to generate photonic crystals, the resulting crystals are prone to defects and reproducibility depends on a variety of 
environmental factors. Overall, there is now a substantial variety of highly attractive fabrication techniques, from which one can chose depending on the application and the materials used. Some of the proposed methods promise easy adaption to mass production as they are compatible with roll-to-roll processing or printing.

\section{Routes towards continuous wave operation}

CW lasing has several advantages over pulsed laser emission, for example in lighting, welding and analytical applications where a constant beam is required over seconds or longer. As described in section 1.3, CW operation in organic gain media is difficult to achieve as triplet excitons accumulate over long excitation periods. In this context, pulsed lasers with pulse durations in the upper millisecond range and lasers operating at repetition rates faster than the typical triplet decay rate are also referred to as (quasi)CW laser as similar problems need to be solved to achieve reliable laser operation.

Even though optical excitation generally generates no triplet excitons (due to spin conservation), a fraction of excited state singlets converts to triplets via intersystem crossing. Once triplets have been generated, they can be often be efficiently excited to a higher lying triplet state via absorption as this process does not violate spin conservation. Triplet absorption can happen at similar wavelength as the singlet emission or may also compete with ground state singlet absorption. Triplet absorption therefore presents a loss mechanism. As triplet excitons decay slowly, they pile up over time, which can stop laser operation after as little as few tens of nanoseconds. In conventional dye lasers this is addressed by continuously circulating the dye solution to replenish fresh luminophores at the excitation site. (The dye solution is not permanently bleached and after triplets have relaxed, the solution can be recycled back to the excitation zone.) In modern optofluidic devices this concept is frequently replicated on smaller scale as described in more detail in section 3.6. 
In organic solid state lasers, a similar mechanism was adapted using a spinning disk coated with a film of an organic laser gain medium -in the reported work a encapsulated laser dye encapsulated in a polymer matrix (e.g. PMMA). ${ }^{270,271}$ An external Fabry-Pérot resonator was aligned so that the disk can rotate freely in the cavity and can be tilted to fulfil the Brewster condition. Due to the spinning of the disk, each position on the disk is only used for a fraction of the time, providing sufficient time for triplets in the gain medium to relax. An alternative strategy to $\mathrm{CW}$ lasing is to introduce pathways for fast triplet relaxation so that the triplet population can be reduced during operation. Naturally, these pathways must not affect singlet excitons. In one example, metal nanoparticles embedded in an organic gain medium have been shown to selectively scavenge triplet excitons via resonant coupling to the plasmon of the metal nanoparticle, thus leading to non-radiative decay of triplets at high rates. Due to their shorter diffusion length, singlet excitons were not affected unless they were generated very close to the metal nanoparticles (see Fig. 14). ${ }^{272}$

(a)

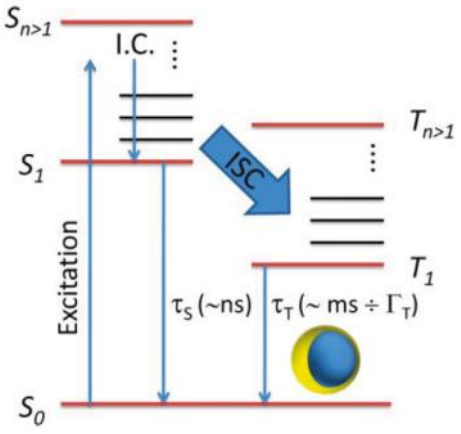

(b)

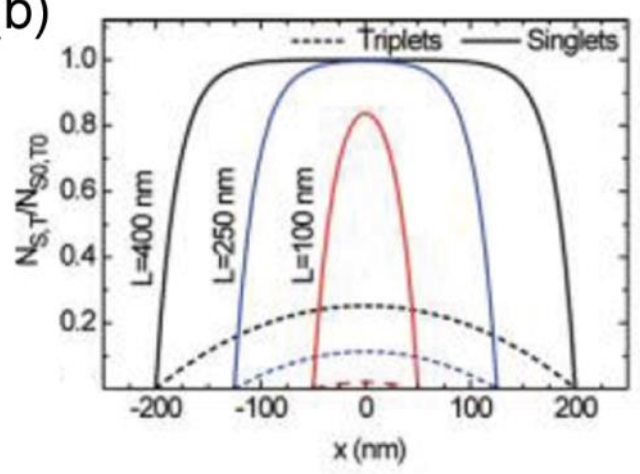

Figure 14. Quenching of triplets using plasmonic particles. (a) Triplet states can be quickly depopulated by coupling to plasmon resonances. Through this plasmon sink, the triplet lifetime $\tau_{T}$ which is usually in the ms to $s$ range can be reduced to $\tau_{T} / \Gamma_{T}$, where the decay rate factor $\Gamma_{T}$ can be on the order of $100 .{ }^{272}$ (b) The density of the plasmonic sinks can be as high as one every $100 \mathrm{~nm}$ without affecting short lived singlet states. Reproduced from $\left[{ }^{272}\right]$. Copyright 2011 American Chemical Society.

Parasitic effects from triplets can also be suppressed by smart molecular design of the gain material. For instance, incorporation of randomly distributed binaphthyl spacer groups in a PFO polymer reduced the conjugation length and allowed spatial and spectral separation of triplets and singlets, somewhat similar 
to the situation with TADF molecules discussed in section 2.1.8. The polymer was spin-coated onto a DFB grating and pumped at different pulse repetition rates. No accumulation of triplet states was observed for repetition rates of up to one pulse every $200 \mathrm{~ns}$ ( $5 \mathrm{MHz}$ ), which may be seen as quasi-CW operation, although triplets in PFO generally have much longer lifetimes of at least $100 \mu \mathrm{s}$. This was explained by an efficient separation of triplets and singlets along the polymer chain: while the PFO domains continue to provide optical gain, triplets were presumably transferred to the binaphthyl domains. The binaphthyl triplets do not show strong absorbance at the wavelength of the pump beam or the lasing wavelength of PFO. ${ }^{273,274}$ Alternatively small molecule triplet scavengers can be blended with high gain conjugated polymers. Anthracene and cyclooctatetraene are known to have short triplet lifetimes and low triplet energies were found to support efficient triplet transfer away from the gain material. ${ }^{275}$ Triplet scavengers were shown to reduce the lasing threshold and increase the maximum duration of laser activity to about $100 \mu \mathrm{s}^{276}$ This represents a major improvement over the pulse lengths of conventional organic lasers which is on the order of $150 \mathrm{~ns}^{277}$

There are materials such as bis(N-carbazole)styryl]biphenyls (similar to CzPV-SBF), which possess optical quantum yields of close to $100 \%$ and a fluorescence lifetime of around $1 \mathrm{~ns}$, when dispersed at $6 \mathrm{wt} \%$ in a bis(carbazolyl)-biphenyl host matrix. When spin coated onto a DFB grating, low threshold lasing $\left(0.25 \mu \mathrm{J} / \mathrm{cm}^{2}\right)$ can be achieved at quasi CW operation with pump pulse repetition rates of $8 \mathrm{MHz}$. Means for triplet relaxation are not required because of insignificant spectral overlap between the emission and triplet absorption of the gain medium. ${ }^{278}$

Apart from triplet states, long lived polaronic states can also contribute significantly to absorption losses. One approach to preventing the formation of polaron states is to increase the intermolecular separation in the solid state. Separation of the emitter units can be achieved by dilution in a semiconducting matrix with larger HOMO-LUMO gap. ${ }^{279}$ The use of cyclodextrin to produce polyrotaxane molecules was also shown to allow ASE under quasi-CW excitation. ${ }^{280}$ These approaches prevent accumulation of polaronic 
states but triplet states will eventually still pile up so that at best one can achieve quasi-CW operation with these.

In summary, several approaches will most likely need to be combined to achieve true CW lasing in solidstate organic lasers. The density of any long lived polaronic and excitonic species needs to be reduced by preventing their generation or finding pathways for their efficient relaxation.

\section{Strategies to overcome the challenges associated with electrical pumping of organic lasers}

The ultimate goal in the field of organic lasers is the creation of an electrically pumped organic laser diode. This would remove the need for an external optical pump source and would enable the creation of thin, conformable or even flexible low-cost lasers. Such devices may well open up a range of new applications for organic lasers. However, there are a number of challenges involved in integrating an OLED or other electroluminescent device architecture into a resonator. At the time of writing this review there is has been no convincing report of an organic laser that can be operated by direct electrical pumping. (There have been a small number of claims of electrically pumped lasing in organic materials but to the best of our knowledge all of these were quickly refuted.) In the following, we discuss different challenges that have been identified by the community and the strategies that were suggested to circumvent them.

\subsection{Absorption by conducting electrodes}

A fundamental difference between electrically pumped and optically pumped lasers is in the presence of the electrodes required to inject charges. These electrodes generally absorb light across a wide range of wavelengths and have larger absorption coefficients than organic semiconductors. While absorption coefficients are particularly large for metallic electrodes, strong absorption also occurs in transparent conductive oxide electrodes. Generally, the interaction of the resonating laser mode with the electrodes 
will reduce the $Q$-factor of the feedback structure and the presence of the electrode means that higher optical gain is required to reach the lasing threshold. This issue is illustrated in Figure $15 \mathrm{a}^{281}$

The problem of absorption losses at the electrodes can be addressed in several ways. One strategy is to increase the distance between the electrodes and the laser-active mode inside the resonator. Several examples of how this can be achieved for DFB feedback structures by increasing the thickness of charge transport layers in OLED type device architectures or by resorting to field effect transistor designs are discussed in section 3.2.3 (also see Figure 15b,c). Alternatively, it was suggested to reduce absorption losses by employing a hybrid transistor/diode structure (Figure $15 \mathrm{~d}$ ). In this design a remote cathode is applied to separate the metallic contact from the organic emission layer. It was demonstrated that the remote electrode device exhibits emission dynamics similar to a standard OLED stack, even when short electrical pulses (down to a few $\mu$ s) were applied, which indicates that this geometry is compatible with the pulsed operation required for organic lasers. ${ }^{282}$

An alternative to increasing the distance between gain material and electrodes is to design a structure that can tolerate the presence of absorbing electrodes without excessive losses. One example of this is an optical waveguide structure with an organic core surrounded by $\mathrm{Al}_{2} \mathrm{O}_{3}$ buffer layers. The thickness of the layers was tuned such that a $\mathrm{TE}_{2}$ mode was supported that had its three field maxima at the center of the organic core and at the center of the buffer layers and its nodes at the interfaces between buffer and core (Figure 15e). This enabled insertion of aluminum doped zinc oxide (AZO) electrode layers between the core and the buffer layers, i.e. at the nodes of the $\mathrm{TE}_{2}$ mode. It was shown that the $\mathrm{TE}_{2}$ mode of this structure showed greatly reduced absorption losses compared to other modes and compared to other waveguide designs with AZO electrodes. ${ }^{283}$ 
(a)

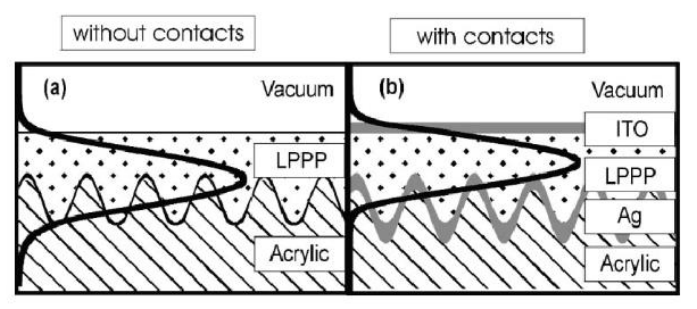

(b)

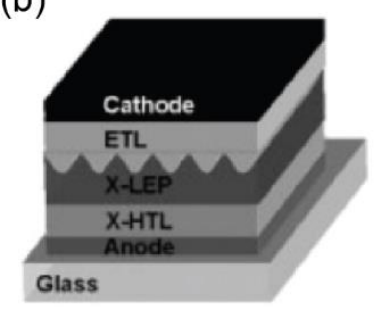

(c)

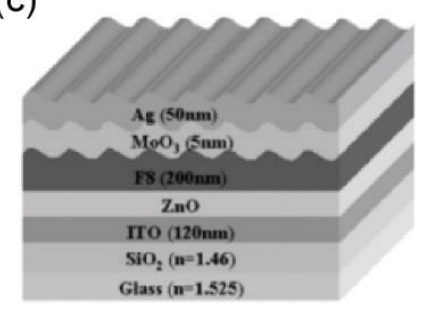

(d)
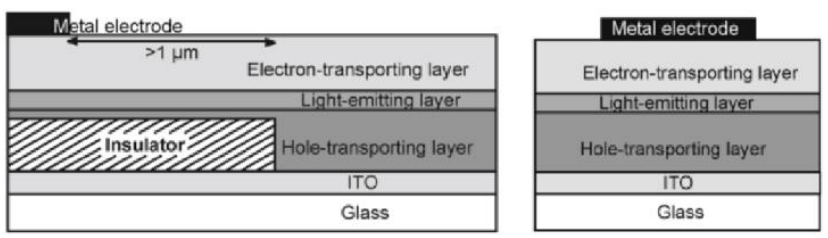

(e)

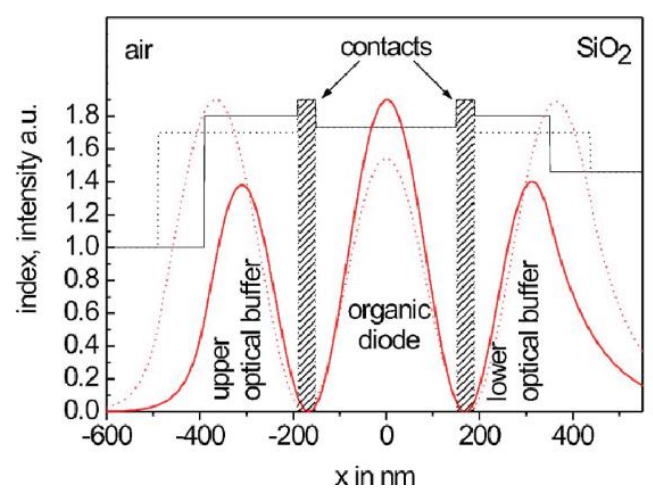

Figure 15. The issue of absorption by electrodes. (a) Mode profile in a light-emitting polymer waveguide with a DFB resonator for a situation with electrodes (right) and without electrodes (left). The overlap of the mode with the electrodes introduces additional absorption and thus is expected to increase the lasing threshold or prevent lasing altogether. ${ }^{281}$ (b) Polymer OLED stack with DFB grating defined in a crosslinkable light emitting polymer layer (X-LEP). By using thick charge transport layers (ETL: electron transport layer, X-HTL: crosslinked hole transport layer), the lasing mode was separated from the electrodes and lasing was achieved under optical pumping. ${ }^{149}$ (c) Similar device concept based on OLED with thick inorganic charge transport layers $\left(\mathrm{MoO}_{3}\right.$ as $\mathrm{HTL}$ and $\mathrm{ZnO}$ as ETL). ${ }^{169}$ (d) Remote metal electrode design (left) and for comparison conventional OLED architecture (right). By creating a region not covered by the metal electrode, absorption losses of a mode guided within the structure can be reduced. ${ }^{282}$ (e) Precise tuning of a guided mode and of the position of electrodes facilitates positioning of electrodes in the nodes of the waveguide mode thus reducing absorption losses without a need for thick charge transport layers. ${ }^{283}$ Reproduced with permission from $\left[{ }^{149}\right.$ ]. Copyright 2010 and $\left[{ }^{169}\right.$ ] Copyright 2009. Wiley-VCH Verlag GmbH \& Co. KGaA. Reprinted from [ ${ }^{281}$ ] and $\left[{ }^{283}\right]$ with the permission of AIP Publishing. Reproduced from $\left[{ }^{282}\right]$ with permission from IEEE. 


\subsection{Increasing charge carrier mobility and reducing excitonic and polaronic losses}

It has been estimated that in order to achieve electrically pumped lasing in an organic laser, current densities of $200 \mathrm{~A} / \mathrm{cm}^{2}$ or higher will be required. ${ }^{284}$ Organic semiconductors generally show much lower charge carrier mobility than their inorganic counterparts. To obtain sufficient current densities, extremely high charge densities are therefore required and the presence of these charges is expected to lead to undesired annihilation processes (in particular singlet-polaron and triplet-polaron quenching). As polarons absorb across a broad spectral range, which often overlaps with the emission of the used gain material, high charge densities may also give rise to additional absorption losses. Optimization of the involved materials towards high mobility reduces the polaron density required to achieve a given current and should help limit singlet-polaron annihilation. In addition, materials engineering can reduce polaron absorption.

An alternative approach is in the drastic reduction of the electrode size of an OLED. One rationale for this is that if electrode dimensions are on the order of the exciton diffusion length, some of the generated excitons escape from the region of current flow to a region with fewer charge carriers. In this way polarons and excitons can be partially separated and exciton-polaron annihilation can be significantly reduced. While conceptually very attractive, the short diffusion lengths of excitons in most organic gain materials (usually well below $50 \mathrm{~nm}$ ) renders practical realization of this approach quite challenging. In practice, are more important consequence of small electrodes may be the reduced Joule heating at high current densities. It has recently been shown experimentally that OLEDs with $50 \mathrm{~nm}$ diameter electrodes retain higher external quantum efficiency at large current densities than larger OLEDs and that current densities (40-fold improvement in critical current density for EQE roll-off). ${ }^{285,286}$ However, we like to point out that for realizing electrically pumped organic lasers, the dimensions of the OLED should probably not be smaller than the lateral extension of the mode supported by the structure (characteristics size, $>\lambda / 2$ ) 
as this would lead to a small overlap between the laser mode and the pumped region of the device which will lead to reduced modal gain.

The relative importance of the annihilation and loss phenomena mentioned above and of a range of other annihilation and loss processes (e.g., singlet-singlet, triplet-triplet and singlet-triplet annihilation and intersystem crossing) was also studied numerically. When taking all annihilation processes into account, the expected threshold current densities increased by more than two-fold (to over $500 \mathrm{~A} / \mathrm{cm}^{2}$ ) compared to a simulation for which these processes were turned off. ${ }^{284}$ The main annihilation process for the investigated system was singlet-polaron quenching, with other annihilation phenomena contributing only to a smaller extent and singlet-singlet-annihilation and intersystem crossing not contributing significantly. This is in contrast to optically pumped lasers, where it was found that depending on the excitation power the dominant quenching mechanisms are either triplet-singlet annihilation or triplet absorption. ${ }^{287}$

A strategy to circumvent mobility problems in organic materials and to thus reduce the charge carrier concentration is to use hybrid organic/inorganic designs. In this way one can exploit the generally higher mobility in inorganic materials but still use the highly efficient organic material for light emission. An example of this approach is a device comprising an organic PFO emissive layer with a DFB grating that is sandwiched between relatively thick inorganic hole-transport $\left(\mathrm{MoO}_{3}\right)$ and electron-transport $(\mathrm{ZnO})$ layers. ${ }^{169}$ In addition to offering increased charge carrier mobility, this design effectively decouples the electrodes from the emissive organic layer and supports a waveguided $\mathrm{TE}_{2}$ mode that is strongly confined to the polyfluorene gain material (see section 6.1). This enabled relatively efficient optically pumped lasing in this structure. While spontaneous electroluminescence was observed under electrical pumping, no indication of electrically pumped lasing was observed. ${ }^{288}$

Although hybrid or purely organic multi-layer structures help confine light to the emissive layer in laser structures and are also widely used in OLEDs, it has been argued that the interfaces between layers also 
introduce losses. To address this issue, non-heterostructure OLEDs with a pure BSB-Cz emission layer and transport layers also based on BSB-CZ but doped with donors or acceptors have been suggested and high current densities of up to $100 \mathrm{~A} / \mathrm{cm}^{2}$ were achieved. ${ }^{289}$

In other work, tetrakis-( $N, N$-diphenylamine)spirobifluorene (S-TAD) were investigated as hole injection and transport layer for organic lasers and it was found that the polaron induced absorption of S-TAD is very low in the $560-660 \mathrm{~nm}$ range, which makes the material particularly useful when combined with red or yellow emitting gain materials. ${ }^{290}$

As discussed in section 2, there has also been a considerable amount of work on improving the charge carrier mobility of organic gain materials themselves. ${ }^{64}$

\subsection{Reducing triplet generation}

Triplet accumulation needs to be inhibited to enable CW operation of organic lasers (see section 5). Reduction of triplet accumulation also plays an important role in electrically pumped lasers because spin statistics predict that upon electrical excitation $75 \%$ of the formed excitons are triplets and only $25 \%$ are singlets. As triplets do not contribute to the lasing process and have long excited state lifetimes, they quickly accumulate in the gain material. Therefore, electrically pumped lasers will either require very short electrical pulses to provide sufficient time for triplet relaxation between pulses or have to involve some other means to reduce the triplet density within the gain material.

Different pathways have been developed to scavenge triplets as has been discussed in section 5 . However, by scavenging triplet excitons, $75 \%$ of the generated excitons remain unused. Small molecules capable of thermally activated delayed fluorescence (TADF, see section 2.1.8) may be able to provide a useful alternative. In a recent study, the TADF molecule ACRXTN was co-doped into an mCBP host, together with a green emitting laser dye (C545T). ${ }^{42}$ This three-component blend had an ASE threshold of $0.8 \mu \mathrm{J} / \mathrm{cm}^{2}$ versus $1.2 \mu \mathrm{J} / \mathrm{cm}^{2}$ for a sample without ACRXTN (in both cases, ASE was generated by the 
C545T molecules; see Figure 16). In time-resolved measurements, the fluorescence from C545T clearly showed a delayed component thus indicating efficient TADF by ACRXTN. The blend was also studied as an emissive layer of an OLED stack. Compared to a reference device without ACRXTN, the efficiency and more importantly the efficiency roll-off were improved by adding the TADF molecule. Systems with TADF based triplet managers now need to be studied in resonator geometries and further studies need to be performed under electrical excitation.

(a)

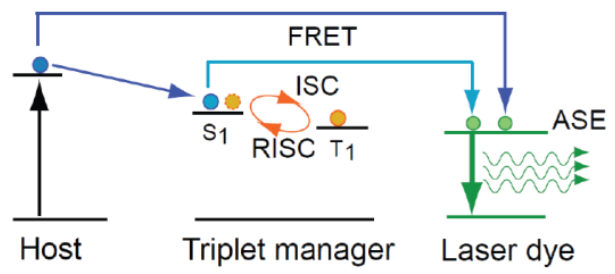

(b)
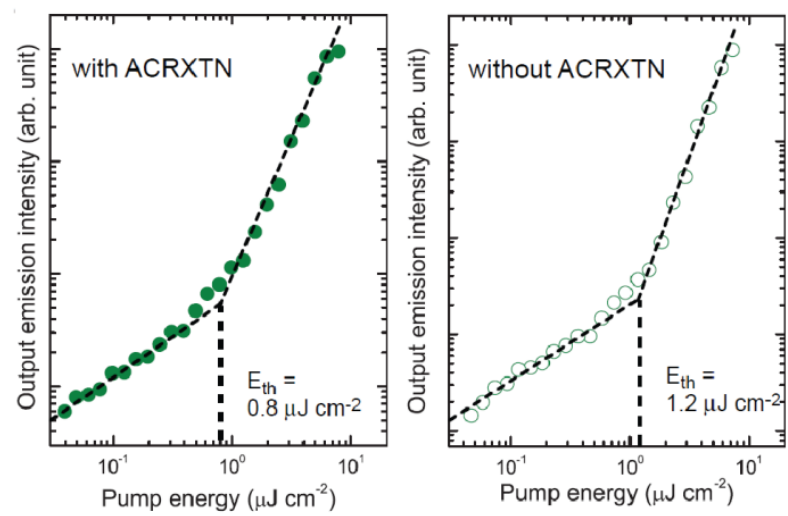

(c)

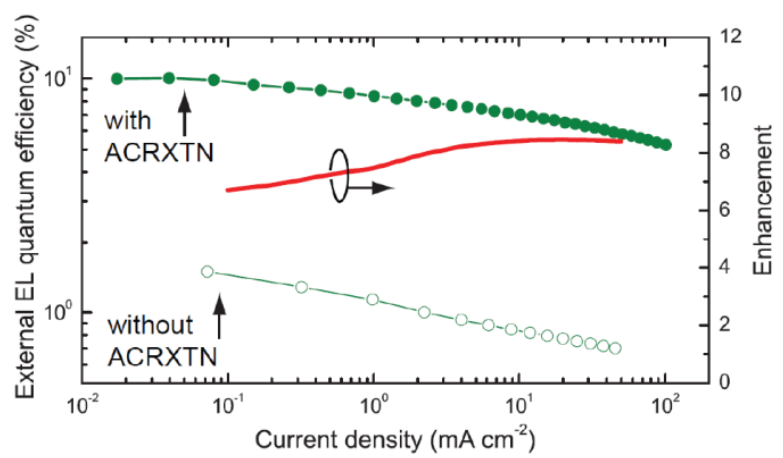

Figure 16: Using TADF materials to circumvent the problem of triplet accumulation. (a) Schematic of the expected energy transfer cascade. The host material is excited, triplets are converted to singlets through RISC on the TADF triplet manager and emission occurs from a co-doped laser dye molecule. (b) ASE characteristics of a thin film with and without the TADF triplet manager ACRXTN. (c) OLED efficiency as a function of applied current density for a device with and without ACRXTN. Reproduced with permission from $\left[{ }^{42}\right]$. Copyright 2015 . Wiley-VCH Verlag GmbH \& Co. KGaA. 


\subsection{Conclusion}

While individual solutions for the problems mentioned above have been developed, electrically pumped lasing in organic media remains unattained. Careful combination of some of the approaches described above - such as triplet recycling, reduction of electrode absorption, reduction of charge carrier densities by using high mobility materials and devices with optimized dimensions - might in the future permit electrically operated laser emission in organic materials. To achieve this, it will be important to follow a holistic approach that considers all processes and effects involved and aims for a global optimum of modal gain and absorption loss.

\section{Outlook}

Organic lasers have come a long way, from the first generation of optical gain in organic semiconductors to organic lasers with record low threshold fluences that are fully integrated with electrical driving schemes, can be fabricated by low-cost nano-imprint or be integrated with live cells. Recent years have seen renewed interest in systematic optimization of laser threshold, with both small molecule and polymer gain materials. It will be exciting to see where the journey of organic lasers is headed over the next decade. Rather than giving a speculative outlook, we will close by first discussing a quickly growing field of organic electronics that is closely related to conventional organic lasers: exciton-polaritons and their condensation. In the second part of this outlook section we will then provide a brief summary of currently emerging applications of organic lasers.

\subsection{Organic polariton lasers}

If the excitonic states in an organic material are strongly coupled to the photonic modes of a surrounding feedback structure, a new type of quasi-particle known as exciton-polaritons is formed. In a simplified picture, exciton-polaritons are formed if photons emitted by an organic material located within a 
feedback structure are more likely to be reabsorbed by the organic material (and to thus again form an exciton) than they are to escape from the structure as free photons. (More precisely, exciton-polaritons need to be introduced to describe the system if the rate of exciton-photon interaction is much larger than the individual decay rates of photons and excitons.) Due to their Bosonic nature, exciton-polaritons do not obey Pauli's exclusion principle (unlike for instance polarons in organic semiconductors). This means that all exciton-polaritons present in the feedback structure can occupy the same quantum state, i.e. they can form a coherent condensate if the polariton density is sufficient. Such a condensate will, however, not be at thermal equilibrium because even in very high $Q$-factor feedback structures a fraction of photons is constantly lost via emission. These leaking photons will retain the coherence of the condensate and thus the emission from an exciton-polariton is in many regards similar to emission from a conventional laser.

Exciton-polariton condensation has been studied for many years in inorganic semiconductor devices. However, as the exciton binding energy in these materials is generally low, condensation is often achieved only at cryogenic temperatures. Organic materials offer a very attractive alternative in this regard and a small number of reports have now been published that demonstrate exciton-polariton condensation in organic systems and at room temperature. Researchers have initially used melt-grown single crystalline anthracene sandwiched between a pair of DBR mirrors. ${ }^{291}$ Recently, this concept was extended to amorphous organic materials. One demonstration used a thermally evaporated film of small molecules $^{117}$ and another report employed a spin-cast ladder-type conjugated polymer referred to as MeLPPP. ${ }^{118}$ In all of these reports, polariton lasing was only observed when the structures were excited with sub 10 ps pulses. Using a biologically-produced green fluorescent protein as gain material, polariton lasing was recently also achieved under ns pumping. ${ }^{86}$ The ability of fluorescent proteins to sustain polariton lasing under these conditions was attributed to their lower exciton-exciton annihilation rates at high exciton densities. Besides being of great interest for studying the physics of quantum condensates, 
polariton lasers have also been hypothesized to offer an easier route to electrically pumped lasing in organic materials. This is because lasing thresholds can in principle be much lower for polariton lasing than for conventional photon lasing. The recent study on polariton lasing from fluorescent proteins demonstrated that the threshold for polariton lasing was 10 -fold lower than the onset of photon lasing. However, the absolute lasing thresholds reported for polariton lasing so far were not lower than the best values reported for conventional organic lasers (120 nJ of $150 \mathrm{fs}$-excitation in Ref. $\left.{ }^{291}\right], 60 \mu \mathrm{J} / \mathrm{cm}^{2}$ of 250 fs-excitation in Ref. $\left[{ }^{117}\right], 500 \mu \mathrm{J} / \mathrm{cm}^{2}$ of 8 ps-excitation in Ref. $\left[{ }^{118}\right]$, and $12 \mathrm{~nJ}$ of 5 ns-excitation in Ref. $\left.\left[{ }^{86}\right]\right)$. It will be exciting to see if future developments of polariton lasers, involving optimized materials, feedback structures and optical pumping schemes can reduce thresholds and thus potentially open a new avenue to electrically pumped organic lasers.

\subsection{Applications of organic lasers}

Due to a number of distinct differences compared with their inorganic counterparts, organic lasers present a highly attractive technology platform for disposable lasers. While organic lasers have relatively low output intensities and can be susceptible to photo-oxidation, they are based on amorphous materials with open molecular morphologies and on resonator structures that are accessible by external cues. This renders organic lasers potentially very useful for analytical systems, e.g. for single use cartridges in bio-medical point-of-care devices at the general practitioner's office or in chemical detection systems that "sniff" minute traces of explosives or drugs to improve security at airports and provide police patrols with on-board forensic analysis.

The organic laser community has indeed already developed several applications along these lines, often based on potentially low cost lab on a chip laser devices that were produced by thermally imprinting grating structures ${ }^{292}$ and that used flexible plastic films as substrates ${ }^{147}$. Laser sources have been

implemented in the form of on-chip DFB lasers ${ }^{293,294}$, DFB lasers on PMMA substrates for microfluidics ${ }^{295}$, 
tunable lasers ${ }^{296}$, micro-goblets ${ }^{297}$ and switchable dye lasers in micro-cavity resonators combined with digital microfuidics ${ }^{209}$. Such microfluidic platform devices have potential applications for on chip excitation of fluorescently labeled antibodies and microspheres or for Raman spectroscopy. ${ }^{298}$

Apart from serving as "simple" illumination sources, organic lasers can also act as versatile chemosensors that can detect minute differences in their chemical environment. In particular, the use of conjugated polymer lasers as sensors for explosives was explored ${ }^{69}$ and it was demonstrated that $\mathrm{DNT}^{299}$ and $\mathrm{TNT}^{69,221,300}$ can be detected at concentrations in the $\mathrm{ppb}$ range. The detection mechanism relies on the fact that interaction between the organic gain material and the explosive (an electron deficient nitroaromatic compound) quenches excitons in the organic material and thus reduces the available optical gain. This resulted in drastic changes in laser output. DFB gratings and random lasers represent ideal resonator geometries for this application as they allow unrestricted access of the analyte to the gain medium and because the laser mode samples the properties of the gain material across a relatively large volume in these resonator geometries.

It was also shown that depending on the organic gain medium, traces of amines ${ }^{301}$, oxygen ${ }^{302}$ and mercury ${ }^{303}$ can also be detected. In addition, organic lasers showed a change in laser emission in laser emission in response to humidity changes and the presence of ethanol vapor. ${ }^{304}$

Further chemicals such as sulfur and cadmium selenide were detected using a Raman spectroscopy scheme that used organic lasers as excitation sources. ${ }^{305}$

A further area where organic lasers are likely to find applications and where proof-of-principle demonstrations have already been reported is bio-sensing, i.e. the detection of small changes to the biochemical environment in the vicinity of the laser. Materials and encapsulants were optimized for operation of organic lasers under water ${ }^{306}$ and it was proposed to use organic lasers as excitation sources for the detection of biomolecules on metallic nanopillar arrays via surface enhanced Raman scattering 
(SERS). ${ }^{307}$ WGM based organic lasers were used to detect small quantities of biomolecules. Bovine serum albumin adsorbed onto organic WGM resonators and the resulting change in the effective refractive index of the circulating mode supported by the cavity caused a shift in the emission wavelength of the laser. ${ }^{182}$ WGM and DFB resonator geometries are both susceptible to changes in the nearby refractive index and depending on the $Q$-factor of the laser, the limit of detection can be on the order of $10^{-5}$ refractive index units. ${ }^{186,308,309}$

WGM resonators were also applied inside cells where they were used as optical bar-codes for cell tracking. This facilitated unambiguous re-identification of cells over prolonged times via the specific laser mode profile of the laser particles taken up. ${ }^{184}$ Intracellular lasers were also used for intracellular sensing and were able to clearly detect when cells were exposed to hypertonic conditions. ${ }^{185}$

\subsection{Conclusion}

Overall, the future appears to look rather bright for organic lasers. It is important, however, to refrain from measuring their success by how well they compete with their inorganic counterparts in wellestablished applications. Instead one should harness the potential of organic for emerging applications, in particular those requiring low cost materials, fast and easy fabrication, and tunability in wavelength or other laser characteristics. Organic lasers have already demonstrated remarkable sensitivity towards their environment, which is largely a result of their mechanical flexibility and open and accessible resonator geometry. Functionalization of organic lasers with tailored chemical and biological recognition motifs holds great potential for further increases in the specificity and sensitivity of organic laser devices. 


\section{References}

(1) Zhu, C.; Liu, L.; Yang, Q.; Lv, F.; Wang, S. Water-Soluble Conjugated Polymers for Imaging, Diagnosis, and Therapy. Chem. Rev. 2012, 112 (8), 4687-4735.

(2) Pecher, J.; Mecking, S. Nanoparticles of Conjugated Polymers. Chem. Rev. 2010, 110 (10), 62606279.

(3) Samuel, I. D. W.; Turnbull, G. A. Organic Semiconductor Lasers. Chem. Rev. 2007, 107 (4), $1272-$ 1295.

(4) Grivas, C.; Pollnau, M. Organic Solid-State Integrated Amplifiers and Lasers. Laser Photonics Rev. 2012, 6 (4), 419-462.

(5) Furumi, S. Recent Advances in Polymer Colloidal Crystal Lasers. Nanoscale 2012, 4 (18), 55645571.

(6) Grimsdale, A. C.; Chan, K. L.; Martin, R. E.; Jokisz, P. G.; Holmes, A. B. Synthesis of Light-Emitting Conjugated Polymers for Applications in Electroluminescent Devices. Chem. Rev. 2009, 109 (3), 897-1091.

(7) Kanibolotsky, A. L.; Perepichka, I. F.; Skabara, P. J. Star-Shaped Pi-Conjugated Oligomers and Their Applications in Organic Electronics and Photonics. Chem. Soc. Rev. 2010, 39 (7), 2695-2728.

(8) Thomas III, S. W.; Guy, D. J.; Swager, T. M. Chemical Sensors Based on Amplifying Fluorescent Conjugated Polymers. Chem. Rev. 2007, 107 (1), 1339-1386.

(9) von Freymann, G.; Kitaev, V.; Lotsch, B. V.; Ozin, G. a. Bottom-up Assembly of Photonic Crystals. Chem. Soc. Rev. 2013, 42, 2528-2554.

(10) Chénais, S.; Forget, S. Recent Advances in Solid-State Organic Lasers. Polym. Int. 2012, 61 (3), 390-406.

(11) Hill, M. T.; Gather, M. C. Advances in Small Lasers. Nat. Photonics 2014, 8 (12), 908-918.

(12) Clark, J.; Lanzani, G. Organic Photonics for Communications. Nat. Photonics 2010, 4 (7), 438-446.

(13) Samuel, I. D. .; Namdas, E. B.; Turnbull, G. A. How to Recognize Lasing. Nat. Photonics 2009, 3 (10), 546-549.

(14) Yokoyama, D.; Nakanotam, H.; Setoguchi, Y.; Moriwake, M.; Ohnishi, D.; Yahiro, M.; Adachi, C. Spectrally Narrow Emission at Cutoff Wavelength from Edge of Electrically Pumped Organic LightEmitting Diodes. Jpn. J. Appl. Phys. 2007, 46 (35), L826-L829.

(15) Yuyama, S.; Nakajima, T.; Yamashita, K.; Oe, K. Solid State Organic Laser Emission at $970 \mathrm{Nm}$ from Dye-Doped Fluorinated-Polyimide Planar Waveguides. Appl. Phys. Lett. 2008, 93 (2), 023306.

(16) Yu, Z.; Li, W.; Hagen, J. A.; Zhou, Y.; Klotzkin, D.; Grote, J. G.; Steckl, A. J. Photoluminescence and Lasing from Deoxyribonucleic Acid (DNA) Thin Films Doped with Sulforhodamine. Appl. Opt. 2007, 46 (9), 1507-1513.

(17) Wallikewitz, B. H.; Nikiforov, G. O.; Sirringhaus, H.; Friend, R. H. A Nanoimprinted, Optically Tuneable Organic Laser. Appl. Phys. Lett. 2012, 100 (17), 10-13.

(18) Calzado, E. M.; Villalvilla, J. M.; Boj, P. G.; Quintana, J. A.; Gómez, R.; Segura, J. L.; Díaz-García, M. A. Effect of Structural Modifications in the Spectral and Laser Properties of Perylenediimide Derivatives. J. Phys. Chem. C 2007, 111 (36), 13595-13605.

(19) Ramírez, M. G.; Morales-Vidal, M.; Navarro-Fuster, V.; Boj, P. G.; Quintana, J. a.; Villalvilla, J. M.; Retolaza, A.; Merino, S.; Díaz-García, M. a. Improved Performance of Perylenediimide-Based Lasers. J. Mater. Chem. C 2013, 1 (6), 1182.

(20) Calzado, E. M.; Ramírez, M. G.; Boj, P. G.; Díaz-García, M. A. Thickness Dependence of Amplified Spontaneous Emission in Low-Absorbing Organic Waveguides. Appl. Opt. 2012, 51 (16), 3287.

(21) Navarro-Fuster, V.; Calzado, E. M.; Boj, P. G.; Quintana, J. a.; Villalvilla, J. M.; Díaz-García, M. A.; Trabadelo, V.; Juarros, A.; Retolaza, A.; Merino, S. Highly Photostable Organic Distributed Feedback Laser Emitting at 573 Nm. Appl. Phys. Lett. 2010, 97 (17), 95-98. 
(22) Yu, Z.; Wu, Y.; Liao, Q.; Zhang, H.; Bai, S.; Li, H.; Xu, Z.; Sun, C.; Wang, X.; Yao, J.; et al. SelfAssembled Microdisk Lasers of Perylenediimides. J. Am. Chem. Soc. 2015, 137 (48), 15105-15111. Esnal, I.; Valois-escamilla, I.; Gómez-Durán, C. F. A.; Urías-Benavides, A.; Betancourt-Mendiola, M.; López-Arbeloa, I.; Bañuelos, J.; García-Moreno, I.; Costela, A.; Peña-Cabrera, E. Blue-toOrange Color-Tunable Laser Emission from Tailored Boron-Dipyrromethene Dyes. ChemPhysChem 2013, 14 (18), 4134-4142.

(24) Mowatt, C.; Morris, S. M.; Song, M. H.; Wilkinson, T. D.; Friend, R. H.; Coles, H. J. Comparison of the Performance of Photonic Band-Edge Liquid Crystal Lasers Using Different Dyes as the Gain Medium. J. Appl. Phys. 2010, 107 (4), 043101.

(25) Gómez-Durán, C. F. A.; García-Moreno, I.; Costela, A.; Martin, V.; Sastre, R.; Bañuelos, J.; López Arbeloa, F.; López Arbeloa, I.; Peña-Cabrera, E. 8-PropargylaminoBODIPY: Unprecedented BlueEmitting Pyrromethene Dye. Synthesis, Photophysics and Laser Properties. Chem. Commun. 2010, 46 (28), 5103-5105.

(26) Forgie, J. C.; Skabara, P. J.; Stibor, I.; Vilela, F.; Vobecka, Z. New Redox Stable Low Band Gap Conjugated Polymer Based on an EDOT-BODIPY-EDOT Repeat Unit The Compound 4, 4-Difluoro4-Borata-3a-Azonia-4a-Aza-S- Indacene, More Commonly Known as BODIPY , Has Enjoyed a High and Rapidly Escalating Level of Interest over. Chem. Mater. 2009, 21 (22), 1784-1786.

(27) Yamao, T.; Yamamoto, K.; Taniguchi, Y.; Miki, T.; Hotta, S. Laser Oscillation in a Highly Anisotropic Organic Crystal with a Refractive Index of 4.0. J. Appl. Phys. 2008, 103 (9), 093115.

(28) Mizuno, H.; Haku, U.; Marutani, Y.; Ishizumi, A.; Yanagi, H.; Sasaki, F.; Hotta, S. Single Crystals of 5,5'-Bis(4'-Methoxybiphenyl-4-YI)-2,2'-Bithiophene for Organic Laser Media. Adv. Mater. 2012, 24 (42), 5744-5749.

(29) Huang, J.; Liu, Q.; Zou, J. H.; Zhu, X. H.; Li, A. Y.; Li, J. W.; Wu, S.; Peng, J.; Cao, Y.; Xia, R.; et al. Electroluminescence and Laser Emission of Soluble Pure Red Fluorescent Molecular Glasses Based on Dithienylbenzothiadiazole. Adv. Funct. Mater. 2009, 19 (18), 2978-2986.

(30) Casado, J.; Hernández, V.; López Navarrete, J. T.; Algarra, M.; da Silva Filho, D. A.; Yamaguchi, S.; Rondão, R.; Seixas de Melo, J. S.; Navarro-Fuster, V.; Boj, P. G.; et al. Amplified Spontaneous Emission in Pentathienoacene Dioxides by Direct Optical Pump and by Energy Transfer: Correlation with Photophysical Parameters. Adv. Opt. Mater. 2013, 1 (8), 588-599.

(31) Calzado, E. M.; Villalvilla, J. M.; Boj, P. G.; Quintana, J. A.; Postigo, P. A.; Díaz-García, M. A. Blue Surface-Emitting Distributed Feedback Lasers Based on TPD-Doped Films. Appl. Opt. 2010, 49 (3), 463-470.

(32) Calzado, E. M.; Boj, P. G.; Díaz-García, M. A. Optimization of the Laser Properties of Polymer Films Doped with N,N'-bis(3-Methylphenyl)-N,N'-diphenylbenzidine. Materials (Basel). 2009, 2 (3), 1288-1304.

(33) Wang, H.; Li, F.; Gao, B.; Xie, Z.; Liu, S.; Wang, C.; Hu, D.; Shen, F.; Xu, Y.; Shang, H.; et al. Doped Organic Crystals with High Efficiency, Color-Tunable Emission toward Laser Application. Cryst. Growth Des. 2009, 9 (11), 4945-4950.

(34) Varghese, S.; Yoon, S. J.; Calzado, E. M.; Casado, S.; Boj, P. G.; Díaz-García, M. A.; Resel, R.; Fischer, R.; Milián-Medina, B.; Wannemacher, R.; et al. Stimulated Resonance Raman Scattering and Laser Oscillation in Highly Emissive Distyrylbenzene-Based Molecular Crystals. Adv. Mater. 2012, 24 (48), 6473-6478.

(35) Varghese, S.; Park, S. K.; Casado, S.; Resel, R.; Wannemacher, R.; Lüer, L.; Park, S. Y.; Gierschner, J. Polymorphism and Amplified Spontaneous Emission in a Dicyano-Distyrylbenzene Derivative with Multiple Trifluoromethyl Substituents: Intermolecular Interactions in Play. Adv. Funct. Mater.

2016, n/a-n/a.

(36) Kabe, R.; Nakanotani, H.; Sakanoue, T.; Yahiro, M.; Adachi, C. Effect of Molecular Morphology on Amplified Spontaneous Emission of Bis-Styrylbenzene Derivatives. Adv. Mater. 2009, 21 (40), 4034-4038. 
(37) Morris, S. M.; Qasim, M. M.; Gardiner, D. J.; Hands, P. J. W.; Castles, F.; Tu, G.; Huck, W. T. S.; Friend, R. H.; Coles, H. J. Liquid Crystalline Chromophores for Photonic Band-Edge Laser Devices. Opt. Mater. (Amst). 2013, 35 (5), 837-842.

(38) Ribierre, J.-C.; Zhao, L.; Inoue, M.; Schwartz, P.-O.; Kim, J.-H.; Yoshida, K.; Sandanayaka, A. S. D.; Nakanotani, H.; Mager, L.; Méry, S.; et al. Low Threshold Amplified Spontaneous Emission and Ambipolar Charge Transport in Non-Volatile Liquid Fluorene Derivatives. Chem. Commun. 2016, $52,3103-3106$.

(39) Kim, J.-H.; Inoue, M.; Zhao, L.; Komino, T.; Seo, S.; Ribierre, J.-C.; Adachi, C. Tunable and Flexible Solvent-Free Liquid Organic Distributed Feedback Lasers. Appl. Phys. Lett. 2015, 106, 053302.

(40) Ribierre, J. C.; Tsiminis, G.; Richardson, S.; Turnbull, G. a.; Samuel, I. D. W.; Barcena, H. S.; Burn, P. L. Amplified Spontaneous Emission and Lasing Properties of Bisfluorene-Cored Dendrimers. Appl. Phys. Lett. 2007, 91 (8), 24-27.

(41) Uoyama, H.; Goushi, K.; Shizu, K.; Nomura, H.; Adachi, C. Highly Efficient Organic Light-Emitting Diodes from Delayed Fluorescence. Nature 2012, 492 (7428), 234-238.

(42) Nakanotani, H.; Furukawa, T.; Adachi, C. Light Amplification in an Organic Solid-State Film with the Aid of Triplet-to-Singlet Upconversion. Adv. Opt. Mater. 2015, 3 (10), 1381-1388.

(43) Nakagawa, T.; Ku, S.-Y.; Wong, K.-T.; Adachi, C. Electroluminescence Based on Thermally Activated Delayed Fluorescence Generated by a Spirobifluorene Donor-acceptor Structure. Chem. Commun. 2012, 48 (77), 9580.

(44) Komino, T.; Nomura, H.; Yahiro, M.; Endo, K.; Adachi, C. Dependence of the Amplified Spontaneous Emission Threshold in Spirofluorene Thin Films on Molecular Orientation. J. Phys. Chem. C 2011, 115 (40), 19890-19896.

(45) Nakanotani, H.; Akiyama, S.; Ohnishi, D.; Moriwake, M.; Yahiro, M.; Yoshihara, T.; Tobita, S.; Adachi, C. Extremely Low-Threshold Amplified Spontaneous Emission of 9,9'-Spirobifluorene Derivatives and Electroluminescence from Field-Effect Transistor Structure. Adv. Funct. Mater. 2007, 17 (14), 2328-2335.

(46) So, H.; Watanabe, H.; Yahiro, M.; Yang, Y.; Oki, Y.; Adachi, C. Highly Photostable DistributedFeedback Polymer Waveguide Blue Laser Using Spirobifluorene Derivatives. Opt. Mater. (Amst). 2011, 33 (6), 755-758.

(47) Niu, Q.; Zhang, Q.; Xu, W.; Jiang, Y.; Xia, R.; Bradley, D. D. C.; Li, D.; Wen, X. Solution-Processed Anthracene-Based Molecular Glasses as Stable Blue-Light-Emission Laser Gain Media. Org. Electron. 2015, 18, 95-100.

(48) Tsiminis, G.; Montgomery, N. a; Kanibolotsky, A. L.; Ruseckas, A.; Perepichka, I. F.; Skabara, P. J.; Turnbull, G. a; Samuel, I. D. W. Laser Characteristics of a Family of Benzene-Cored Star-Shaped Oligofluorenes. Semicond. Sci. Technol. 2012, 27 (9), 094005.

(49) Skabara, P. J.; Pethrick, R. a.; Mackintosh, A. R.; Kanibolotsky, A. L.; Dawson, M. D. Amplified Spontaneous Emission in Free-Standing Membranes Incorporating Star-Shaped Monodisperse $\pi$ Conjugated Truxene Oligomers. J. Opt. 2010, 12, 035503.

(50) Wang, Y.; Tsiminis, G.; Yang, Y.; Ruseckas, A.; Kanibolotsky, A. L.; Perepichka, I. F.; Skabara, P. J.; Turnbull, G. a.; Samuel, I. D. W. Broadly Tunable Deep Blue Laser Based on a Star-Shaped Oligofluorene Truxene. Synth. Met. 2010, 160 (13-14), 1397-1400.

(51) Tsiminis, G.; Wang, Y.; Shaw, P. E.; Kanibolotsky, A. L.; Perepichka, I. F.; Dawson, M. D.; Skabara, P. J.; Turnbull, G. a.; Samuel, I. D. W. Low-Threshold Organic Laser Based on an Oligofluorene Truxene with Low Optical Losses. Appl. Phys. Lett. 2009, 94 (24), 243304. Lai, W. Y.; Xia, R.; Bradley, D. D. C.; Huang, W. 2,3,7,8,12,13-Hexaaryltruxenes: An OrthoSubstituted Multiarm Design and Microwave-Accelerated Synthesis toward Starburst Macromolecular Materials with Well-Defined Pi-Delocalization. Chem. - A Eur. J. 2010, 16 (28), 8471-8479.

(53) Lai, W. Y.; Xia, R.; He, Q. Y.; Levermore, P. a.; Huang, W.; Bradley, D. D. C. Enhanced Solid-State 
Luminescence and Low-Threshold Lasing from Starburst Macromolecular Materials. Adv. Mater. 2009, 21 (3), 355-360.

(54) Xia, R.; Lai, W.-Y.; Levermore, P. a.; Huang, W.; Bradley, D. D. C. Low-Threshold DistributedFeedback Lasers Based on Pyrene-Cored Starburst Molecules with 1,3,6,8-Attached Oligo(9,9Dialkylfluorene) Arms. Adv. Funct. Mater. 2009, 19 (17), 2844-2850.

(55) Kanibolotsky, A. L.; Vilela, F.; Forgie, J. C.; Elmasly, S. E. T.; Skabara, P. J.; Zhang, K.; Tieke, B.; McGurk, J.; Belton, C. R.; Stavrinou, P. N.; et al. Well-Defined and Monodisperse Linear and StarShaped Quaterfluorene-DPP Molecules: The Significance of Conjugation and Dimensionality. Adv. Mater. 2011, 23 (18), 2093-2097.

(56) Stavrinou, P. N.; Ryu, G.; Campoy-Quiles, M.; Bradley, D. D. C. The Change in Refractive Index of poly(9,9-Dioctylfluorene) due to the Adoption of the $\beta$-Phase Chain Conformation. J. Phys. Condens. Matter 2007, 19 (46), 466107.

(57) Song, M. H.; Wenger, B.; Friend, R. H. Tuning the Wavelength of Lasing Emission in Organic Semiconducting Laser by the Orientation of Liquid Crystalline Conjugated Polymer. J. Appl. Phys. 2008, 104 (3), 033107.

(58) Ryu, G.; Stavrinou, P. N.; Bradley, D. D. C. Spatial Patterning of the $\beta$-Phase in Poly(9,9Dioctylfluorene): A Metamaterials-Inspired Molecular Conformation Approach to the Fabrication of Polymer Semiconductor Optical Structures. Adv. Funct. Mater. 2009, 19 (20), 3237-3242.

(59) Kuehne, A. J. C.; MacKintosh, A. R.; Pethrick, R. a. $\beta$-Phase Formation in a Crosslinkable Poly(9,9Dihexylfluorene). Polymer (Guildf). 2011, 52 (24), 5538-5542.

(60) Kuehne, A. J. C.; Kaiser, M.; Mackintosh, A. R.; Wallikewitz, B. H.; Hertel, D.; Pethrick, R. A.; Meerholz, K. Sub-Micrometer Patterning of Amorphous- and $\beta$-Phase in a Crosslinkable Poly $(9,9-$ Dioctylfluorene): Dual-Wavelength Lasing from a Mixed-Morphology Device. Adv. Funct. Mater. 2011, 21 (13), 2564-2570.

(61) McDowell, J. J.; Maier-Flaig, F.; Wolf, T. J. a; Unterreiner, A. N.; Lemmer, U.; Ozin, G. Synthesis and Application of Photolithographically Patternable Deep Blue Emitting poly(3,6-Dimethoxy-9,9Dialkylsilafluorene)s. ACS Appl. Mater. Interfaces 2014, 6 (1), 83-93.

(62) Tsiminis, G.; Ruseckas, A.; Samuel, I. D. W.; Turnbull, G. a. A Two-Photon Pumped Polyfluorene Laser. Appl. Phys. Lett. 2009, 94 (25).

(63) Wu, P. L.; Feng, X. J.; Tam, H. L.; Wong, M. S.; Cheah, K. W. Efficient Three-Photon Excited Deep Blue Photoluminescence and Lasing of Diphenylamino and 1,2,4-Triazole Endcapped Oligofluorenes. J. Am. Chem. Soc. 2009, 131 (3), 886-887.

(64) Yap, B. K.; Xia, R.; Campoy-Quiles, M.; Stavrinou, P. N.; Bradley, D. D. C. Simultaneous Optimization of Charge-Carrier Mobility and Optical Gain in Semiconducting Polymer Films. Nat. Mater. 2008, 7 (5), 376-380.

(65) Xia, R.; Heliotis, G.; Hou, Y.; Bradley, D. D. C. Fluorene-Based Conjugated Polymer Optical Gain Media. Org. Electron. 2003, 4, 165-177.

(66) Amarasinghe, D.; Ruseckas, A.; Vasdekis, A. E.; Turnbull, G. a.; Samuel, I. D. W. High-Gain Broadband Solid-State Optical Amplifier Using a Semiconducting Copolymer. Adv. Mater. 2009, 21 (1), 107-110.

(67) Laquai, F.; Mishra, A. K.; Müllen, K.; Friend, R. H. Amplified Spontaneous Emission of Poly (LadderType Phenylene)s - The Influence of Photophysical Properties on ASE Thresholds. Adv. Funct. Mater. 2008, 18 (20), 3265-3275.

(68) Kim, H.; Schulte, N.; Zhou, G.; Müllen, K.; Laquai, F. A High Gain and High Charge Carrier Mobility Indenofluorene-Phenanthrene Copolymer for Light Amplification and Organic Lasing. Adv. Mater. 2011, 23 (7), 894-897.

(69) Rose, A.; Zhu, Z.; Madigan, C. F.; Swager, T. M.; Bulović, V. Sensitivity Gains in Chemosensing by Lasing Action in Organic Polymers. Nature 2005, 434 (7035), 876-879.

(70) Chen, Y.; Herrnsdorf, J.; Guilhabert, B.; Kanibolotsky, A. L.; MacKintosh, A. R.; Wang, Y.; Pethrick, 
R. a.; Gu, E.; Turnbull, G. a.; Skabara, P. J.; et al. Laser Action in a Surface-Structured Free-Standing Membrane Based on a Pi-Conjugated Polymer-Composite. Org. Electron. physics, Mater. Appl. 2011, 12 (1), 62-69.

(71) Chen, Y.; Herrnsdorf, J.; Guilhabert, B.; Zhang, Y.; Kanibolotsky, A. L.; Skabara, P. J.; Gu, E.; Laurand, N.; Dawson, M. D. Modification of Emission Wavelength in Organic Random Lasers Based on Photonic Glass. Org. Electron. physics, Mater. Appl. 2012, 13 (7), 1129-1135.

(72) Scotognella, F.; Puzzo, D. P.; Zavelani-Rossi, M.; Clark, J.; Sebastian, M.; Ozin, G. a.; Lanzani, G. Two-Photon Poly(phenylenevinylene) DFB Laser. Chem. Mater. 2011, 23 (3), 805-809.

(73) Martini, I. B.; Craig, I. M.; Molenkamp, W. C.; Miyata, H.; Tolbert, S. H.; Schwartz, B. J. Controlling Optical Gain in Semiconducting Polymers with Nanoscale Chain Positioning and Alignment. Nat. Nanotechnol. 2007, 2 (10), 647-652.

(74) Xia, R.; Stavrinou, P. N.; Bradley, D. D. C.; Kim, Y. Efficient Optical Gain Media Comprising Binary Blends of poly(3-Hexylthiophene) and poly(9,9-Dioctylfluorene-Co-Benzothiadiazole). J. Appl. Phys. 2012, 111, 123107.

(75) Heliotis, G.; Xia, R.; Bradley, D. D. C.; Turnbull, G. a.; Samuel, I. D. W.; Andrew, P.; Barnes, W. L. Two-Dimensional Distributed Feedback Lasers Using a Broadband, Red Polyfluorene Gain Medium. J. Appl. Phys. 2004, 96 (12), 6959-6965.

(76) Wallikewitz, B. H.; Hertel, D.; Meerholz, K. Cross-Linkable Polyspirobifluorenes: A Material Class Featuring Good OLED Performance and Low Amplified Spontaneous Emission Thresholds. Chem. Mater. 2009, 21 (13), 2912-2919.

(77) Yu, L.; Liu, J.; Hu, S.; He, R.; Yang, W.; Wu, H.; Peng, J.; Xia, R.; Bradley, D. D. C. Red, Green, and Blue Light-Emitting Polyfluorenes Containing a Dibenzothiophene- $S, S$-Dioxide Unit and Efficient High-Color-Rendering-Index White-Light-Emitting Diodes Made Therefrom. Adv. Funct. Mater. 2013, 23 (35), 4366-4376.

(78) Saiga, Y. O. Extraction, Purification and Properties of Aequorin , a Bioluminescent Protein from the Luminous. 1887, 1353 (165), 223-239.

(79) Gather, M. C.; Yun, S. H. Single-Cell Biological Lasers. Nat. Photonics 2011, 5, 406-410.

(80) Gather, M. C.; Yun, S. H. Lasing from Escherichia Coli Bacteria Genetically Programmed to Express Green Fluorescent Protein. Opt. Lett. 2011, 36 (16), 3299-3301.

(81) Gather, M. C.; Yun, S. H. Bio-Optimized Energy Transfer in Densely Packed Fluorescent Protein Enables near-Maximal Luminescence and Solid-State Lasers. Nat. Commun. 2014, 5, 5722.

(82) Jonáš, A.; Aas, M.; Karadag, Y.; Manioğlu, S.; Anand, S.; McGloin, D.; Bayraktar, H.; Kiraz, A. In Vitro and in Vivo Biolasing of Fluorescent Proteins Suspended in Liquid Microdroplet Cavities. Lab Chip 2014, 14 (16), 3093-3100.

(83) Chen, Q.; Zhang, X.; Sun, Y.; Ritt, M.; Sivaramakrishnan, S.; Fan, X. Highly Sensitive Fluorescent Protein FRET Detection Using Optofluidic Lasers. Lab Chip 2013, 13 (14), 2679-2681.

(84) Chen, Q.; Ritt, M.; Sivaramakrishnan, S.; Sun, Y.; Fan, X. Optofluidic Lasers with a Single Molecular Layer of Gain. Lab Chip 2014, 14 (24), 4590-4595.

(85) Dietrich, C. P.; Höfling, S.; Gather, M. C. Multi-State Lasing in Self-Assembled Ring-Shaped Green Fluorescent Protein Microcavities. Appl. Phys. Lett. 2014, 105, 233702.

(86) Dietrich, C. P.; Steude, A.; Tropf, L.; Schubert, M.; Kronenberg, Nils, M.; Ostermann, K.; Höfling, S.; Gather, M. C. An Exciton-Polariton Laser Based on Biologically Produced Fluorescent Protein. arXiv 2016, 1601.06983.

(87) Wu, X.; Chen, Q.; Sun, Y.; Fan, X. Bio-Inspired Optofluidic Lasers with Luciferin. Appl. Phys. Lett. 2013, 102 (20), 2014-2017.

(88) Nizamoglu, S.; Gather, M. C.; Yun, S. H. All-Biomaterial Laser Using Vitamin and Biopolymers. Adv. Mater. 2013, 25 (41), 5943-5947.

(89) Vannahme, C.; Maier-Flaig, F.; Lemmer, U.; Kristensen, A. Single-Mode Biological Distributed Feedback Laser. Lab Chip 2013, 13 (14), 2675-2678. 
(90) Jeon, N. J.; Noh, J. H.; Kim, Y. C.; Yang, W. S.; Ryu, S.; Seok, S. Il. Solvent Engineering for HighPerformance Inorganic-Organic Hybrid Perovskite Solar Cells. Nat. Mater. 2014, 13, 897-903.

(91) Schulz, P.; Edri, E.; Kirmayer, S.; Hodes, G.; Cahen, D.; Kahn, A. Interface Energetics in OrganoMetal Halide Perovskite-Based Photovoltaic Cells. Energy Environ. Sci. 2014, 7 (4), 1377.

(92) Zhang, H.; Shallcross, R. C.; Li, N.; Stubhan, T.; Hou, Y.; Chen, W.; Ameri, T.; Turbiez, M.; Armstrong, N. R.; Brabec, C. J. Overcoming Electrode-Induced Losses in Organic Solar Cells by Tailoring a Quasi-Ohmic Contact to Fullerenes via Solution-Processed Alkali Hydroxide Layers. Adv. Energy Mater. 2016.

(93) Kim, Y.-H.; Cho, H.; Heo, J. H.; Kim, T.-S.; Myoung, N.; Lee, C.-L.; Im, S. H.; Lee, T.-W. Multicolored Organic/Inorganic Hybrid Perovskite Light-Emitting Diodes. Adv. Mater. 2015, 27 (7), 1248-1254.

(94) Zhang, S.; Audebert, P.; Wei, Y.; Lauret, J.-S.; Galmiche, L.; Deleporte, E. Synthesis and Optical Properties of Novel Organic-inorganic Hybrid UV (R-NH3)2PbCl4 Semiconductors. J. Mater. Chem. 2011, 21 (2), 466.

(95) Motta, C.; El-Mellouhi, F.; Sanvito, S. Charge Carrier Mobility in Hybrid Halide Perovskites. Sci. Rep. 2015, 5, 12746.

(96) Shi, D.; Adinolfi, V.; Comin, R.; Yuan, M.; Alarousu, E.; Buin, A.; Chen, Y.; Hoogland, S.; Rothenberger, A.; Katsiev, K.; et al. Low Trap-State Density and Long Carrier Diffusion in Organolead Trihalide Perovskite Single Crystals. Science (80-. ). 2015, 347 (6221), 519-522.

(97) Nie, W.; Tsai, H.; Asadpour, R.; Neukirch, A. J.; Gupta, G.; Crochet, J. J.; Chhowalla, M.; Tretiak, S.; Alam, M. A.; Wang, H.; et al. High-Efficiency Solution-Processed Perovskite Solar Cells with Millimeter-Scale Grains. Science (80-. ). 2015, 347 (6221), 522-525.

(98) Xing, G.; Mathews, N.; Lim, S. S.; Yantara, N.; Liu, X.; Sabba, D.; Grätzel, M.; Mhaisalkar, S.; Sum, T. C. Low-Temperature Solution-Processed Wavelength-Tunable Perovskites for Lasing. Nat. Mater. 2014, 13 (5), 476-480.

(99) Deschler, F.; Price, M.; Pathak, S.; Klintberg, L. E.; Jarausch, D. D.; Higler, R.; Hüttner, S.; Leijtens, T.; Stranks, S. D.; Snaith, H. J.; et al. High Photoluminescence Efficiency and Optically Pumped Lasing in Solution-Processed Mixed Halide Perovskite Semiconductors. J. Phys. Chem. Lett. 2014, 5 (8), 1421-1426.

(100) Sutherland, B. R.; Hoogland, S.; Adachi, M. M.; Wong, C. T. O.; Sargent, E. H. Conformal Organohalide Perovskites Enable Lasing on Spherical Resonators. ACS Nano 2014, 8 (10), 1094710952.

(101) Sun, W.; Wang, K.; Gu, Z.; Xiao, S.; Song, Q. Tunable Perovskite Microdisk Lasers. Nanoscale 2016.

(102) Zhang, Q.; Ha, S. T.; Liu, X.; Sum, T. C.; Xiong, Q. Room-Temperature near-Infrared High-Q Perovskite Whispering-Gallery Planar Nanolasers. Nano Lett. 2014, 14 (10), 5995-6001.

(103) Zhu, H.; Fu, Y.; Meng, F.; Wu, X.; Gong, Z.; Ding, Q.; Gustafsson, M. V.; Trinh, M. T.; Jin, S.; Zhu, X.Y. Lead Halide Perovskite Nanowire Lasers with Low Lasing Thresholds and High Quality Factors. Nat. Mater. 2015, 14, 636-643.

(104) Nikolaenko, A. E.; Cass, M.; Bourcet, F.; Mohamad, D.; Roberts, M. Thermally Activated Delayed Fluorescence in Polymer: A New Route toward Highly Efficient Solution Processable OLEDs. Adv. Mater. 2015, 27 (44), 7236-7240.

(105) Lee, T. W.; Park, O. O.; Choi, D. H.; Cho, H. N.; Kim, Y. C. Low-Threshold Blue Amplified Spontaneous Emission in a Statistical Copolymer and Its Blend. Appl. Phys. Lett. 2002, 81 (3), 424426.

(106) Karnutsch, C.; Pflumm, C.; Heliotis, G.; DeMello, J. C.; Bradley, D. D. C.; Wang, J.; Weimann, T.; Haug, V.; Gärtner, C.; Lemmer, U. Improved Organic Semiconductor Lasers Based on a MixedOrder Distributed Feedback Resonator Design. Appl. Phys. Lett. 2007, 90 (13), 131104.

(107) Tessler, N.; Denton, G. J.; Friend, R. H. Lasing from Conjugated-Polymer Microcavities. Nature 1996, 382, 695-697.

(108) Bulovic, V.; Kozlov, V. G.; Khalfin, V. B.; Forrest, S. R. Transform-Limited, Narrow-Linewidth Lasing 
Action in Organic Semiconductor Microcavities. Science (80-. ). 1998, 279, 553-555.

(109) Schütte, B.; Gothe, H.; Hintschich, S. I.; Sudzius, M.; Fröb, H.; Lyssenko, V. G.; Leo, K. Continuously Tunable Laser Emission from a Wedge-Shaped Organic Microcavity. Appl. Phys. Lett. 2008, 92 (16), 163309.

(110) Mischok, a.; Lemke, F.; Reinhardt, C.; Brückner, R.; Zakhidov, a. a.; Hintschich, S. I.; Fröb, H.; Lyssenko, V. G.; Leo, K. Dispersion Tomography of an Organic Photonic-Wire Microcavity. Appl. Phys. Lett. 2013, 103 (18), 183302.

(111) Sudzius, M.; Langner, M.; Hintschich, S. I.; Lyssenko, V. G.; Fröb, H.; Leo, K. Multimode Laser Emission from Laterally Confined Organic Microcavities. Appl. Phys. Lett. 2009, 94 (6), 92-95.

(112) Langner, M.; Sudzius, M.; Fröb, H.; Lyssenko, V. G.; Leo, K. Selective Excitation of Laser Modes in an Organic Photonic Dot Microcavity. Appl. Phys. Lett. 2009, 95 (9), 2007-2010.

(113) Brückner, R.; Zakhidov, a. a.; Scholz, R.; Sudzius, M.; Hintschich, S. I.; Fröb, H.; Lyssenko, V. G.; Leo, K. Phase-Locked Coherent Modes in a Patterned Metal-organic Microcavity. Nat. Photonics 2012, 6 (5), 322-326.

(114) Mischok, A.; Brückner, R.; Sudzius, M.; Reinhardt, C.; Lyssenko, V. G.; Fröb, H.; Leo, K. Photonic Confinement in Laterally Structured Metal-Organic Microcavities. Appl. Phys. Lett. 2014, 105, 051108.

(115) Nizamoglu, S.; Lee, K.-B.; Gather, M. C.; Kim, K. S.; Jeon, M.; Kim, S.; Humar, M.; Yun, S.-H. A Simple Approach to Biological Single-Cell Lasers Via Intracellular Dyes. Adv. Opt. Mater. 2015, 3 (9), 1197-1200.

(116) Humar, M.; Gather, M. C.; Yun, S.-H. Cellular Dye Lasers: Lasing Thresholds and Sensing in a Planar Resonator. Opt. Express 2015, 23 (21), 27865.

(117) Daskalakis, K. S.; Maier, S. a; Murray, R.; Kéna-Cohen, S. Nonlinear Interactions in an Organic Polariton Condensate. Nat. Mater. 2014, 13 (3), 271-278.

(118) Plumhof, J. D.; Stoeferle, T.; Mai, L.; Scherf, U.; Mahrt, R. Room-Temperature Bose-Einstein Condensation of Cavity Exciton-polaritons in a Polymer. Nat. Mater. 2014, 13 (4), 247-252.

(119) Kéna-Cohen, S.; Forrest, S. R. Room-Temperature Polariton Lasing in an Organic Single-Crystal Microcavity. Nat. Photonics 2010, 4 (6), 371-375.

(120) Yamashita, K.; Nakahata, T.; Hayakawa, T.; Sakurai, Y.; Yamao, T.; Yanagi, H.; Hotta, S. Vertical Cavity Surface Emitting Lasing from Cyano-Substituted Thiophene/phenylene Co-Oligomer Single Crystals. Appl. Phys. Lett. 2014, 104 (25), 253301.

(121) Oh, H. J.; Gather, M. C.; Song, J.-J. J.; Yun, S. H. Lasing from Fluorescent Protein Crystals. Opt. Express 2014, 22 (25), 31411-31416.

(122) Song, H.; Singer, K.; Lott, J.; Wu, Y.; Zhou, J.; Andrews, J.; Baer, E.; Hiltner, A.; Weder, C. Continuous Melt Processing of All-Polymer Distributed Feedback Lasers. J. Mater. Chem. 2009, 19 (40), 7520.

(123) Comoretto, G. C. and F. S. and G. L. and S. D. S. and M. Z.-R. and D. Lasing from All-Polymer Microcavities. Laser Phys. Lett. 2014, 11 (3), 35804.

(124) Mhibik, O.; Leang, T.; Siove, A.; Forget, S.; Chénais, S. Broadly Tunable (440-670 Nm) Solid-State Organic Laser with Disposable Capsules. Appl. Phys. Lett. 2013, 102 (4), 041112.

(125) Zhao, Z.; Mhibik, O.; Nafa, M.; Chénais, S.; Forget, S. High Brightness Diode-Pumped Organic SolidState Laser. Appl. Phys. Lett. 2015, 106 (5), 051112.

(126) Forget, S.; Rabbani-Haghighi, H.; Diffalah, N.; Siove, A.; Chénais, S. Tunable Ultraviolet VerticallyEmitting Organic Laser. Appl. Phys. Lett. 2011, 98 (13), 131102.

(127) Rabbani-Haghighi, H.; Forget, S.; Chénais, S.; Siove, A. Highly Efficient, Diffraction-Limited Laser Emission from a Vertical External-Cavity Surface-Emitting Organic Laser. Opt. Lett. 2010, 35 (12), 1968-1970.

(128) Yamashita, K.; Kitanobou, a.; Ito, M.; Fukuzawa, E.; Oe, K. Solid-State Organic Laser Using SelfWritten Active Waveguide with in-Line Fabry-Pérot Cavity. Appl. Phys. Lett. 2008, 92 (14), 
143305.

(129) Stöferle, T.; Moll, N.; Wahlbrink, T.; Bolten, J.; Mollenhauer, T.; Scherf, U.; Mahrt, R. F. Ultracompact Silicon/polymer Laser with an Absorption-Insensitive Nanophotonic Resonator. Nano Lett. 2010, 10 (9), 3675-3678.

(130) Chang, W.; Wang, A.; Murarka, A.; Akselrod, G. M.; Packard, C.; Lang, J. H.; Bulović, V. Electrically Tunable Organic Vertical-Cavity Surface-Emitting Laser. Appl. Phys. Lett. 2014, 105 (7), 073303.

(131) Chakaroun, M.; Coens, a; Fabre, N.; Gourdon, F.; Solard, J.; Fischer, a; Boudrioua, a; Lee, C. C. Optimal Design of a Microcavity Organic Laser Device under Electrical Pumping. Opt. Express 2011, 19 (2), 493-505.

(132) Coens, A.; Chakaroun, M.; Fischer, a P. A.; Lee, M. W.; Boudrioua, A.; Geffroy, B.; Vemuri, G. Experimental Optimization of the Optical and Electrical Properties of a Half-Wavelength-Thick Organic Hetero-Structure in a Micro-Cavity. Opt. Express 2012, 20 (28), 29252-29259.

(133) Riedl, T.; Rabe, T.; Johannes, H. H.; Kowalsky, W.; Wang, J.; Weimann, T.; Hinze, P.; Nehls, B.; Farrell, T.; Scherf, U. Tunable Organic Thin-Film Laser Pumped by an Inorganic Violet Diode Laser. Appl. Phys. Lett. 2006, 88, 241116.

(134) Vasdekis, A. E.; Tsiminis, G.; Ribierre, J.-C.; O’ Faolain, L.; Krauss, T. F.; Turnbull, G. A.; Samuel, I. D. W. Diode Pumped Distributed Bragg Reflector Lasers Based on a Dye-to-Polymer Energy Transfer Blend. Opt. Express 2006, 14 (20), 9211-9216.

(135) Karnutsch, C.; Haug, V.; Gaertner, C.; Lemmer, U.; Farrell, T.; Nehls, B. S.; Scherf, U.; Wang, J.; Weimann, T.; Heliotis, G.; et al. Low Threshold Blue Conjugated Polymer DFB Lasers. In Conference on Lasers and Electro-Optics/Quantum Electronics and Laser Science Conference and Photonic Applications Systems Technologies; Technical Digest (CD); Optical Society of America: Long Beach, California , 2006; p CFJ3.

(136) Yang, Y.; Turnbull, G. A.; Samuel, I. D. W. Hybrid Optoelectronics: A Polymer Laser Pumped by a Nitride Light-Emitting Diode. Appl. Phys. Lett. 2008, 92, 163306.

(137) Tsiminis, G.; Wang, Y.; Kanibolotsky, A. L.; Inigo, A. R.; Skabara, P. J.; Samuel, I. D. W.; Turnbull, G. a. Nanoimprinted Organic Semiconductor Laser Pumped by a Light-Emitting Diode. Adv. Mater. 2013, 25 (20), 2826-2830.

(138) Wang, Y.; Tsiminis, G.; Kanibolotsky, A. L.; Skabara, P. J.; Samuel, I. D. W.; Turnbull, G. A. Nanoimprinted Polymer Lasers with Threshold below 100 W / Cm 2 Using Mixed-Order Distributed Feedback Resonators. Opt. Express 2013, 21 (12), 9211-9216.

(139) Herrnsdorf, J.; Wang, Y.; Mckendry, J. J. D.; Gong, Z.; Massoubre, D.; Guilhabert, B.; Tsiminis, G.; Turnbull, G. A.; Samuel, I. D. W.; Laurand, N.; et al. Micro-LED Pumped Polymer Laser: A Discussion of Future Pump Sources for Organic Lasers. Laser Photonics Rev. 2013, 7 (6), 10651078.

(140) Navarro-Fuster, V.; Vragovic, I.; Calzado, E. M.; Boj, P. G.; Quintana, J. a.; Villalvilla, J. M.; Retolaza, A.; Juarros, A.; Otaduy, D.; Merino, S.; et al. Film Thickness and Grating Depth Variation in Organic Second-Order Distributed Feedback Lasers. J. Appl. Phys. 2012, 112, 043104.

(141) Vannahme, C.; Smith, C. L. C.; Brokner Christiansen, M.; Kristensen, A. Emission Wavelength of Multilayer Distributed Feedback Dye Lasers. Appl. Phys. Lett. 2012, 101 (15), 151123.

(142) Martins, E. R.; Wang, Y.; Kanibolotsky, A. L.; Skabara, P. J.; Turnbull, G. a.; Samuel, I. D. W. LowThreshold Nanoimprinted Lasers Using Substructured Gratings for Control of Distributed Feedback. Adv. Opt. Mater. 2013, 1 (8), 563-566.

(143) Klinkhammer, S.; Woggon, T.; Geyer, U.; Vannahme, C.; Dehm, S.; Mappes, T.; Lemmer, U. A Continuously Tunable Low-Threshold Organic Semiconductor Distributed Feedback Laser Fabricated by Rotating Shadow Mask Evaporation. Appl. Phys. B Lasers Opt. 2009, 97 (4), 787791.

(144) Klinkhammer, S.; Liu, X.; Huska, K.; Shen, Y.; Vanderheiden, S.; Valouch, S.; Vannahme, C.; Bräse, S.; Mappes, T.; Lemmer, U. Continuously Tunable Solution-Processed Organic Semiconductor DFB 
Lasers Pumped by Laser Diode. Opt. Express 2012, 20 (6), 6357.

(145) Stroisch, M.; Woggon, T.; Teiwes-Morin, C.; Klinkhammer, S.; Forberich, K.; Gombert, a; Gerken, M.; Lemmer, U. Intermediate High Index Layer for Laser Mode Tuning in Organic Semiconductor Lasers. Opt. Express 2010, 18 (6), 5890-5895.

(146) Görrn, P.; Lehnhardt, M.; Kowalsky, W.; Riedl, T.; Wagner, S. Elastically Tunable Self-Organized Organic Lasers. Adv. Mater. 2011, 23 (7), 869-872.

(147) Guilhabert, B.; Laurand, N.; Herrnsdorf, J.; Chen, Y.; Kanibolotsky, a. L.; Orofino, C.; Skabara, P. J.; Dawson, M. D. Mechanically Flexible Organic Semiconductor Laser Array. IEEE Photonics J. 2012, 4 (3), 684-690.

(148) Schauer, S.; Liu, X.; Worgull, M.; Lemmer, U.; Hölscher, H. Shape-Memory Polymers as Flexible Resonator Substrates for Continuously Tunable Organic DFB Lasers. Opt. Mater. Express 2015, 5 (3), 576.

(149) Wallikewitz, B. H.; de la Rosa, M.; Kremer, J. H.-W. M.; Hertel, D.; Meerholz, K. A Lasing Organic Light-Emitting Diode. Adv. Mater. 2010, 22 (4), 531-534.

(150) Baumann, K.; Stöferle, T.; Moll, N.; Raino, G.; Mahrt, R. F.; Wahlbrink, T.; Bolten, J.; Scherf, U. Design and Optical Characterization of Photonic Crystal Lasers with Organic Gain Material. J. Opt. 2010, 12 (6), 065003.

(151) Baumann, K.; Stöferle, T.; Moll, N.; Mahrt, R. F.; Wahlbrink, T.; Bolten, J.; Mollenhauer, T.; Moormann, C.; Scherf, U. Organic Mixed-Order Photonic Crystal Lasers with Ultrasmall Footprint. Appl. Phys. Lett. 2007, 91 (17), 171108.

(152) Nazirizadeh, Y.; Lemmer, U.; Gerken, M. Experimental Quality Factor Determination of GuidedMode Resonances in Photonic Crystal Slabs. Appl. Phys. Lett. 2008, 93, 261110.

(153) Liang, Y.; Peng, C.; Sakai, K.; Iwahashi, S.; Noda, S. Three-Dimensional Coupled-Wave Model for Square-Lattice Photonic Crystal Lasers with Transverse Electric Polarization: A General Approach. Phys. Rev. B - Condens. Matter Mater. Phys. 2011, 84, 195119.

(154) Sakai, K.; Miyai, E.; Noda, S. Two-Dimensional Coupled Wave Theory for Square-Lattice PhotonicCrystal Lasers with TM-Polarization. Opt. Express 2007, 15 (7), 3981-3990.

(155) Sakai, K.; Yue, J.; Noda, S. Coupled-Wave Model for Triangular-Lattice Photonic Crystal with Transverse Electric Polarization. Opt. Express 2008, 16 (9), 6033-6040.

(156) Peng, C.; Liang, Y.; Sakai, K.; Iwahashi, S.; Noda, S. Three-Dimensional Coupled-Wave Theory Analysis of a Centered-Rectangular Lattice Photonic Crystal Laser with a Transverse-Electric-like Mode. Phys. Rev. B 2012, 86 (3), 35108.

(157) Zhen, B.; Chua, S.-L.; Lee, J.; Rodriguez, A. W.; Liang, X.; Johnson, S. G.; Joannopoulos, J. D.; Soljacic, M.; Shapira, O. Enabling Enhanced Emission and Low-Threshold Lasing of Organic Molecules Using Special Fano Resonances of Macroscopic Photonic Crystals. Proc. Natl. Acad. Sci. U. S. A. 2013, 110 (34), 13711-13716.

(158) Nishijima, Y.; Ueno, K.; Juodkazis, S.; Mizeikis, V.; Misawa, H.; Maeda, M.; Minaki, M. Tunable Single-Mode Photonic Lasing from Zirconia Inverse Opal Photonic Crystals. Opt. Express 2008, 16 (18), 13676-13684.

(159) Nishijima, Y.; Ueno, K.; Juodkazis, S.; Mizeikis, V.; Fujiwara, H.; Sasaki, K.; Misawa, H. Lasing with Well-Defined Cavity Modes in Dye-Infiltrated Silica Inverse Opals. Opt. Express 2009, 17 (4), 29762983.

(160) Castles, F.; Day, F. V.; Morris, S. M.; Ko, D.-H.; Gardiner, D. J.; Qasim, M. M.; Nosheen, S.; Hands, P. J. W.; Choi, S. S.; Friend, R. H.; et al. Blue-Phase Templated Fabrication of Three-Dimensional Nanostructures for Photonic Applications. Nat. Mater. 2012, 11 (7), 599-603.

(161) Shi, L.-T.; Jin, F.; Zheng, M.-L.; Dong, X.-Z.; Chen, W.-Q.; Zhao, Z.-S.; Duan, X.-M. Threshold Optimization of Polymeric Opal Photonic Crystal Cavity as Organic Solid-State Dye-Doped Laser. Appl. Phys. Lett. 2011, 98 (9), 093304.

(162) Scotognella, F.; Puzzo, D. P.; Monguzzi, A.; Wiersma, D. S.; Maschke, D.; Tubino, R.; Ozin, G. a. 
Nanoparticle One-Dimensional Photonic-Crystal Dye Laser. Small 2009, 5 (18), 2048-2052.

(163) Gwinner, M. C.; Khodabakhsh, S.; Song, M. H.; Schweizer, H.; Giessen, H.; Sirringhaus, H. Integration of a Rib Waveguide Distributed Feedback Structure into a Light-Emitting Polymer Field-Effect Transistor. Adv. Funct. Mater. 2009, 19 (9), 1360-1370.

(164) Yamao, T.; Sakurai, Y.; Terasaki, K.; Shimizu, Y.; Jinnai, H.; Hotta, S. Current-Injected SpectrallyNarrowed Emissions from an Organic Transistor. Adv. Mater. 2010, 22 (33), 3708-3712.

(165) Capelli, R.; Toffanin, S.; Generali, G.; Usta, H.; Facchetti, A.; Muccini, M. Organic Light-Emitting Transistors with an Efficiency That Outperforms the Equivalent Light-Emitting Diodes. Nat. Mater. 2010, 9 (6), 496-503.

(166) Hsu, B. B. Y.; Duan, C.; Namdas, E. B.; Gutacker, A.; Yuen, J. D.; Huang, F.; Cao, Y.; Bazan, G. C.; Samuel, I. D. W.; Heeger, A. J. Control of Efficiency, Brightness, and Recombination Zone in LightEmitting Field Effect Transistors. Adv. Mater. 2012, 24 (9), 1171-1175.

(167) Sawabe, K.; Imakawa, M.; Nakano, M.; Yamao, T.; Hotta, S.; Iwasa, Y.; Takenobu, T. CurrentConfinement Structure and Extremely High Current Density in Organic Light-Emitting Transistors. Adv. Mater. 2012, 24 (46), 6141-6146.

(168) Gwinner, M. C.; Kabra, D.; Roberts, M.; Brenner, T. J. K.; Wallikewitz, B. H.; McNeill, C. R.; Friend, R. H.; Sirringhaus, H. Highly Efficient Single-Layer Polymer Ambipolar Light-Emitting Field-Effect Transistors. Adv. Mater. 2012, 24 (20), 2728-2734.

(169) Song, M. H.; Kabra, D.; Wenger, B.; Friend, R. H.; Snaith, H. J. Optically-Pumped Lasing in Hybrid Organic-Inorganic Light-Emitting Diodes. Adv. Funct. Mater. 2009, 19 (13), 2130-2136.

(170) Gather, M. C.; Ventsch, F.; Meerholz, K. Embedding Organic Light-Emitting Diodes into Channel Waveguide Structures. Adv. Mater. 2008, 20 (10), 1966-1971.

(171) Camposeo, A.; Benedetto, F. Di; Stabile, R.; Neves, A. a R.; Cingolani, R.; Pisignano, D. Laser Emission from Electrospun Polymer Nanofibers. Small 2009, 5 (5), 562-566.

(172) Di Benedetto, F.; Camposeo, A.; Pagliara, S.; Mele, E.; Persano, L.; Stabile, R.; Cingolani, R.; Pisignano, D. Patterning of Light-Emitting Conjugated Polymer Nanofibres. Nat. Nanotechnol. 2008, 3 (10), 614-619.

(173) O'Carroll, D.; Lieberwirth, I.; Redmond, G.; Carroll, D. O. Microcavity Effects and Optically Pumped Lasing in Single Conjugated Polymer Nanowires. Nat. Nanotechnol. 2007, 2 (3), 180-184.

(174) Zhang, C.; Zou, C. L.; Yan, Y.; Hao, R.; Sun, F. W.; Han, Z. F.; Zhao, Y. S.; Yao, J. Two-Photon Pumped Lasing in Single-Crystal Organic Nanowire Exciton Polariton Resonators. J. Am. Chem. Soc. 2011, 133 (19), 7276-7279.

(175) Chiasera, A.; Dumeige, Y.; Féron, P.; Ferrari, M.; Jestin, Y.; Nunzi Conti, G.; Pelli, S.; Soria, S.; Righini, G. C. Spherical Whispering-Gallery-Mode Microresonators. Laser Photon. Rev. 2010, 4 (3), 457-482.

(176) He, L.; Özdemir, Ş. K.; Yang, L. Whispering Gallery Microcavity Lasers. Laser Photon. Rev. 2013, 7 (1), 60-82.

(177) Grudinin, I. S.; Ilchenko, V. S.; Maleki, L. Ultrahigh Optical Q Factors of Crystalline Resonators in the Linear Regime. Phys. Rev. A 2006, 74 (6), 63806.

(178) Tang, S. K. Y.; Li, Z.; Abate, A. R.; Agresti, J. J.; Weitz, D. A.; Psaltis, D.; Whitesides, G. M. A MultiColor Fast-Switching Microfluidic Droplet Dye Laser. Lab Chip 2009, 9 (19), 2767-2771.

(179) Aas, M.; Jonáš, A.; Kiraz, A. Lasing in Optically Manipulated, Dye-Doped Emulsion Microdroplets. Opt. Commun. 2013, 290, 183-187.

(180) Özelci, E.; Aas, M.; Jonáš, A.; Kiraz, A. Optofluidic FRET Microlasers Based on Surface-Supported Liquid Microdroplets. Laser Phys. Lett. 2014, 11, 045802.

(181) Karadag, Y.; Aas, M.; Jonáš, A.; Anand, S.; McGloin, D.; Kiraz, A. Dye Lasing in Optically Manipulated Liquid Aerosols. Opt. Lett. 2013, 38 (10), 1669-1671.

(182) Francois, A.; Himmelhaus, M. Whispering Gallery Mode Biosensor Operated in the Stimulated Emission Regime. Appl. Phys. Lett. 2009, 94 (3), 031101. 
(183) François, A.; Riesen, N.; Ji, H.; Afshar V., S.; Monro, T. M. Polymer Based Whispering Gallery Mode Laser for Biosensing Applications. Appl. Phys. Lett. 2015, 106 (3), 031104.

(184) Schubert, M.; Steude, A.; Liehm, P.; Kronenberg, N. M.; Karl, M.; Campbell, E. C.; Powis, S. J.; Gather, M. C. Lasing within Live Cells Containing Intracellular Optical Microresonators for Barcode-Type Cell Tagging and Tracking. Nano Lett. 2015, 15 (8), 5647-5652.

(185) Humar, M.; Hyun Yun, S. Intracellular Microlasers. Nat. Photonics 2015, 9, 572-577.

(186) Wienhold, T.; Kraemmer, S.; Wondimu, S. F.; Siegle, T.; Bog, U.; Weinzierl, U.; Schmidt, S.; Becker, H.; Kalt, H.; Mappes, T.; et al. All-Polymer Photonic Sensing Platform Based on Whispering-Gallery Mode Microgoblet Lasers. Lab Chip 2015, 15 (18), 3800-3806.

(187) Grossmann, T.; Schleede, S.; Hauser, M.; Christiansen, M. B.; Vannahme, C.; Eschenbaum, C.; Klinkhammer, S.; Beck, T.; Fuchs, J.; Nienhaus, G. U.; et al. Low-Threshold Conical Microcavity Dye Lasers. Appl. Phys. Lett. 2010, 97 (6), 063304.

(188) Grossmann, T.; Schleede, S.; Hauser, M.; Christiansen, M. B.; Vannahme, C.; Eschenbaum, C.; Klinkhammer, S.; Beck, T.; Fuchs, J.; Nienhaus, G.; et al. Lasing in Dye-Doped High-Q Conical Polymeric Microcavities. Proc. SPIE 2011, 7913, 79130Y.

(189) Klinkhammer, S.; Großmann, T.; Lüll, K.; Hauser, M.; Vannahme, C.; Mappes, T.; Kalt, H.; Lemmer, U. Diode-Pumped Organic Semiconductor Microcone Laser. IEEE Photonics Technol. Lett. 2011, 23 (8), 489-491.

(190) Grossmann, T.; Klinkhammer, S.; Hauser, M.; Floess, D.; Beck, T.; Vannahme, C.; Mappes, T.; Lemmer, U.; Kalt, H. Strongly Confined, Low-Threshold Laser Modes in Organic Semiconductor Microgoblets. Opt. Express 2011, 19 (10), 10009-10016.

(191) Sasaki, F.; Kobayashi, S.; Haraichi, S.; Fujiwara, S.; Bando, K.; Masumoto, Y.; Hotta, S. Microdisk and Microring Lasers of Thiophene-Phenylene Co-Oligomers Embedded in Si/SiO2 Substrates. Adv. Mater. 2007, 19 (21), 3653-3655.

(192) Rotschild, C.; Tomes, M.; Mendoza, H.; Andrew, T. L.; Swager, T. M.; Carmon, T.; Baldo, M. a. Cascaded Energy Transfer for Efficient Broad-Band Pumping of High-Quality, Micro-Lasers. Adv. Mater. 2011, 23 (27), 3057-3060.

(193) Grossmann, T.; Wienhold, T.; Bog, U.; Beck, T.; Friedmann, C.; Kalt, H.; Mappes, T. Polymeric Photonic Molecule Super-Mode Lasers on Silicon. Light Sci. Appl. 2013, 2 (5), e82.

(194) Shopova, S. I.; Zhou, H.; Fan, X.; Zhang, P. Optofluidic Ring Resonator Based Dye Laser. Appl. Phys. Lett. 2007, 90 (22), 221101.

(195) Duong Ta, V.; Chen, R.; Ma, L.; Jun Ying, Y.; Dong Sun, H. Whispering Gallery Mode Microlasers and Refractive Index Sensing Based on Single Polymer Fiber. Laser Photon. Rev. 2013, 7 (1), 133139.

(196) Ta, V. D.; Chen, R.; Sun, H. D. Self-Assembled Flexible Microlasers. Adv. Mater. 2012, 24 (10), OP60-OP64.

(197) Yoshioka, H.; Ota, T.; Chen, C.; Ryu, S.; Yasui, K.; Oki, Y. Extreme Ultra-Low Lasing Threshold of Full-Polymeric Fundamental Microdisk Printed with Room-Temperature Atmospheric Ink-Jet Technique. Sci. Rep. 2015, 5, 10623.

(198) Qian, H.; Markman, B. D.; Giebink, N. C. Vector Vortex Beam Emission from Organic Semiconductor Microlasers. Appl. Phys. Lett. 2013, 103, 161110.

(199) Zhan, X. P.; Ku, J. F.; Xu, Y. X.; Zhang, X. L.; Zhao, J.; Fang, W.; Xu, H. L.; Sun, H. B. Unidirectional Lasing from a Spiral-Shaped Microcavity of Dye-Doped Polymers. IEEE Photonics Technol. Lett. 2015, 27 (3), 311-314.

(200) Humar, M.; Ravnik, M.; Pajk, S.; Musevic, I. Electrically Tunable Liquid Crystal Optical Microresonators. Nat. Photonics 2009, 3 (10), 595-600.

(201) Stolyarov, A. M.; Wei, L.; Shapira, O.; Sorin, F.; Chua, S. L.; Joannopoulos, J. D.; Fink, Y. Microfluidic Directional Emission Control of an Azimuthally Polarized Radial Fibre Laser. Nat. Photonics 2012, 6 (4), 229-233. 
(202) Xu, Z.; Liao, Q.; Shi, Q.; Zhang, H.; Yao, J.; Fu, H. Low-Threshold Nanolasers Based on SlabNanocrystals of H-Aggregated Organic Semiconductors. Adv. Mater. 2012, 24 (35), 216-220.

(203) Liao, Q.; Jin, X.; Zhang, H.; Xu, Z.; Yao, J.; Fu, H. An Organic Microlaser Array Based on a Lateral Microcavity of a Single J-Aggregation Microbelt. Angew. Chemie - Int. Ed. 2015, No. 973, 70377041.

(204) Wang, X.; Liao, Q.; Kong, Q.; Zhang, Y.; Xu, Z.; Lu, X.; Fu, H. Whispering-Gallery-Mode Microlaser Based on Self-Assembled Organic Single-Crystalline Hexagonal Microdisks. Angew. Chemie - Int. Ed. 2014, 53 (23), 5863-5867.

(205) Wang, X.; Liao, Q.; Lu, X.; Li, H.; Xu, Z.; Fu, H. Shape-Engineering of Self-Assembled Organic Single Microcrystal as Optical Microresonator for Laser Applications. Sci. Rep. 2014, 4, 7011.

(206) Fang, H. H.; Ding, R.; Lu, S. Y.; Yang, Y. De; Chen, Q. D.; Feng, J.; Huang, Y. Z.; Sun, H. B. Whispering-Gallery Mode Lasing from Patterned Molecular Single-Crystalline Microcavity Array. Laser Photonics Rev. 2013, 7 (2), 281-288.

(207) Bisri, S. Z.; Takenobu, T.; Yomogida, Y.; Shimotani, H.; Yamao, T.; Hotta, S.; Iwasa, Y. High Mobility and Luminescent Efficiency in Organic Single-Crystal Light-Emitting Transistors. Adv. Funct. Mater. 2009, 19 (11), 1728-1735.

(208) Coles, D. M.; Trichet, A. A. P.; Dolan, P. R.; Taylor, R. A.; Vallance, C.; Smith, J. M. Diffusion-Driven Continuous-Wave-Pumped Organic Dye Lasers. Laser Photonics Rev. 2015, 9 (5), 538-544.

(209) Kuehne, A. J. C.; Gather, M. C.; Eydelnant, I. a.; Yun, S.-H.; Weitz, D. a.; Wheeler, A. R. A Switchable Digital Microfluidic Droplet Dye-Laser. Lab Chip 2011, 11 (21), 3716-3719.

(210) Wu, X.; Oo, M. K. K.; Reddy, K.; Chen, Q.; Sun, Y.; Fan, X. Optofluidic Laser for Dual-Mode Sensitive Biomolecular Detection with a Large Dynamic Range. Nat Commun 2014, 5, 3779.

(211) Song, W.; Vasdekis, A. E.; Li, Z.; Psaltis, D. Low-Order Distributed Feedback Optofluidic Dye Laser with Reduced Threshold. Appl. Phys. Lett. 2009, 94, 051117.

(212) Gersborg-Hansen, M.; Kristensen, A. Tunability of Optofluidic Distributed Feedback Dye Lasers. Opt. Express 2007, 15 (1), 137-142.

(213) Song, W.; Vasdekis, A. E.; Li, Z.; Psaltis, D. Optofluidic Evanescent Dye Laser Based on a Distributed Feedback Circular Grating. Appl. Phys. Lett. 2009, 94 (16), 161110.

(214) Karl, M.; Whitworth, G. L.; Schubert, M.; Dietrich, C. P.; Samuel, I. D. W.; Turnbull, G. A.; Gather, M. C. Optofluidic Distributed Feedback Lasers with Evanescent Pumping : Reduced Threshold and Angular Dispersion Analysis Optofluidic Distributed Feedback Lasers with Evanescent Pumping : Reduced Threshold and Angular Dispersion Analysis. Appl Phys Lett 2016, 261101 (108), 261101.

(215) Tulek, a.; Polson, R. C.; Vardeny, Z. V. Naturally Occurring Resonators in Random Lasing of $\pi-$ Conjugated Polymer Films. Nat. Phys. 2010, 6 (4), 303-310.

(216) Tulek, a; Vardeny, Z. V. Studies of Random Laser Action in $\pi$-Conjugated Polymers. J. Opt. 2010, $12,024008$.

(217) Gottardo, S.; Sapienza, R.; García, P. D.; Blanco, A.; Wiersma, D. S.; López, C. Resonance-Driven Random Lasing. Nat. Photonics 2008, 2 (7), 429-432.

(218) Polson, R. C.; Vardeny, Z. V. Spatially Mapping Random Lasing Cavities. Opt. Lett. 2010, 35 (16), 2801-2803.

(219) Kéna-Cohen, S.; Stavrinou, P. N.; Bradley, D. D. C.; Maier, S. A. Random Lasing in Low Molecular Weight Organic Thin Films. Appl. Phys. Lett. 2011, 99, 041114.

(220) Wiersma, D. S.; Article, R. The Physics and Applications of Random Lasers. Nat. Phys. 2008, 4 (5), 359-367.

(221) Deng, C.; He, Q.; He, C.; Shi, L.; Cheng, J.; Lin, T. Conjugated Polymer-Titania Nanoparticle Hybrid Films: Random Lasing Action and Ultrasensitive Detection of Explosive Vapors. J. Phys. Chem. $B$ 2010, 114 (13), 4725-4730.

(222) Redding, B.; Choma, M. a.; Cao, H. Speckle-Free Laser Imaging Using Random Laser Illumination. Nat. Photonics 2012, 6 (6), 355-359. 
(223) Mahler, L.; Tredicucci, A.; Beltram, F.; Walther, C.; Faist, J.; Beere, H. E.; Ritchie, D. A.; Wiersma, D. S. Quasi-Periodic Distributed Feedback Laser. Nat. Photonics 2010, 4 (3), 165-169.

(224) Berini, P.; De Leon, I. Surface Plasmon-polariton Amplifiers and Lasers. Nat. Photonics 2011, 6 (1), 16-24.

(225) Leosson, K. Optical Amplification of Surface Plasmon Polaritons: Review. J. Nanophotonics 2012, 6 (1), 61801.

(226) Gather, M. C.; Meerholz, K.; Danz, N.; Leosson, K. Net Optical Gain in a Plasmonic Waveguide Embedded in a Fluorescent Polymer. Nat. Photonics 2010, 4 (7), 457-461.

(227) De Leon, I.; Berini, P. Amplification of Long-Range Surface Plasmons by a Dipolar Gain Medium. Nat. Photonics 2010, 4 (6), 382-387.

(228) Kéna-Cohen, S.; Stavrinou, P. N.; Bradley, D. D. C.; Maier, S. A. Confined Surface PlasmonPolariton Amplifiers. Nano letters. American Chemical Society February 25, 2013, pp 1323-1329.

(229) Slovinsky, I.; Stefansson, G. K.; Kossoy, A.; Leosson, K. Propagation Loss of Long-Range Surface Plasmon Polariton Gold Stripe Waveguides in the Thin-Film Limit. Plasmonics 2013, 8 (4), 16131619.

(230) Leosson, K.; Ingason, A. S.; Agnarsson, B.; Kossoy, A.; Olafsson, S.; Gather, M. C. Ultra-Thin Gold Films on Transparent Polymers. Nanophotonics 2013, 2 (1), 3-11.

(231) Schwab, T.; Schubert, S.; Hofmann, S.; Fröbel, M.; Fuchs, C.; Thomschke, M.; Müller-Meskamp, L.; Leo, K.; Gather, M. C. Highly Efficient Color Stable Inverted White Top-Emitting OLEDs with UltraThin Wetting Layer Top Electrodes. Adv. Opt. Mater. 2013, 1 (10), 707-713.

(232) Keshmarzi, E. K.; Niall Tait, R.; Berini, P. Long-Range Surface Plasmon Single-Mode Laser Concepts. J. Appl. Phys. 2012, 112, 063115.

(233) Paul, A.; Zhen, Y. R.; Wang, Y.; Chang, W. S.; Xia, Y.; Nordlander, P.; Link, S. Dye-Assisted Gain of Strongly Confined Surface Plasmon Polaritons in Silver Nanowires. Nano Lett. 2014, 14 (6), 36283633.

(234) Noginov, M. A.; Zhu, G.; Belgrave, A. M.; Bakker, R.; Shalaev, V. M.; Narimanov, E. E.; Stout, S.; Herz, E.; Suteewong, T.; Wiesner, U. Demonstration of a Spaser-Based Nanolaser. Nature 2009, 460 (7259), 1110-1112.

(235) Meng, X.; Kildishev, A. V.; Fujita, K.; Tanaka, K.; Shalaev, V. M. Wavelength-Tunable Spasing in the Visible. Nano Lett. 2013, 13 (9), 4106-4112.

(236) Peng, B.; Zhang, Q.; Liu, X.; Ji, Y.; Demir, H. V.; Huan, C. H. A.; Sum, T. C.; Xiong, Q. FluorophoreDoped Core-Multishell Spherical Plasmonic Nanocavities: Resonant Energy Transfer toward a Loss Compensation. ACS Nano 2012, 6 (7), 6250-6259.

(237) Suh, J. Y.; Kim, C. H.; Zhou, W.; Huntington, M. D.; Co, D. T.; Wasielewski, M. R.; Odom, T. W. Plasmonic Bowtie Nanolaser Arrays. Nano Lett. 2012, 12 (11), 5769-5774.

(238) Zhou, W.; Dridi, M.; Suh, J. Y.; Kim, C. H.; Co, D. T.; Wasielewski, M. R.; Schatz, G. C.; Odom, T. W. Lasing Action in Strongly Coupled Plasmonic Nanocavity Arrays. Nat. Nanotechnol. 2013, 8 (7), 506-511.

(239) Meng, X.; Liu, J.; Kildishev, A. V.; Shalaev, V. M. Highly Directional Spaser Array for the Red Wavelength Region. Laser Photonics Rev. 2014, 8 (6), 896-903.

(240) Fisslthaler, E.; Sezen, M.; Plank, H.; BI ümel, A.; Sax, S.; Grogger, W.; List, E. J. . Direct SubMicrometer-Patterning of Conjugated Polymers and Polymer Light-Emitting Devices by Electron Beam Lithography. Macromol. Chem. Phys. 2010, 211 (13), 1402-1407.

(241) Credgington, D.; Fenwick, O.; Charas, A.; Morgado, J.; Suhling, K.; Cacialli, F. High-Resolution Scanning Near-Field Optical Lithography of Conjugated Polymers. Adv. Funct. Mater. 2010, 20 (17), 2842-2847.

(242) Woggon, T.; Kleiner, T.; Punke, M.; Lemmer, U. Nanostructuring of Organic-Inorganic Hybrid Materials for Distributed Feedback Laser Resonators by Two-Photon Polymerization. Opt. Express 2009, 17 (4), 2500-2507. 
(243) Perevedentsev, A.; Sonnefraud, Y.; Belton, C. R.; Sharma, S.; Cass, A. E. G.; Maier, S. a; Kim, J.; Stavrinou, P. N.; Bradley, D. D. C. Dip-Pen Patterning of poly(9,9-Dioctylfluorene) ChainConformation-Based Nano-Photonic Elements. Nat. Commun. 2015, 6, 1-9.

(244) Ramírez, M. G.; Quintana, J. a.; Villalvilla, J. M.; Boj, P. G.; Retolaza, A.; Merino, S.; Díaz-García, M. a. Perylenediimide-Based Distributed Feedback Lasers with Holographic Relief Gratings on Dichromated Gelatine. J. Appl. Phys. 2013, 114 (3).

(245) Ramírez, M. G.; Villalvilla, J. M.; Quintana, J. a.; Boj, P. G.; Díaz-García, M. a. Distributed Feedback Lasers Based on Dichromated Poly(vinyl Alcohol) Reusable Surface-Relief Gratings. Opt. Mater. Express 2014, 4 (4), 733.

(246) Stroisch, M.; Woggon, T.; Lemmer, U.; Bastian, G.; Violakis, G.; Pissadakis, S. Organic Semiconductor Distributed Feedback Laser Fabricated by Direct Laser Interference Ablation. Opt. Express 2007, 15 (7), 3968-3973.

(247) Zhai, T.; Zhang, X.; Pang, Z.; Dou, F. Direct Writing of Polymer Lasers Using Interference Ablation. Adv. Mater. 2011, 23 (16), 1860-1864.

(248) Ge, C.; Lu, M.; Jian, X.; Tan, Y.; Cunningham, B. T. Large-Area Organic Distributed Feedback Laser Fabricated by Nanoreplica Molding and Horizontal Dipping. Opt. Express 2010, 18 (12), 1298012991.

(249) Reboud, V.; Romero-Vivas, J.; Lovera, P.; Kehagias, N.; Kehoe, T.; Redmond, G.; Sotomayor Torres, C. M. Lasing in Nanoimprinted Two-Dimensional Photonic Crystal Band-Edge Lasers. Appl. Phys. Lett. 2013, 102 (7).

(250) Christiansen, M. B.; Smith, C. L. C.; Buß, T.; Xiao, S.; Mortensen, N. a; Kristensen, A. Nanoimprinted Polymer Photonic Crystal Dye Lasers. Micro-Optics 2010 2010, 7716 (1), 771600.

(251) Ramirez, M. G.; Boj, P. G.; Navarro-Fuster, V.; Vragovic, I.; Villalvilla, J. M.; Alonso, I.; Trabadelo, V.; Merino, S.; Díaz-García, M. A. Efficient Organic Distributed Feedback Lasers with Imprinted Active Films. Opt. Express 2011, 19 (23), 22443-22454.

(252) Trabadelo, V.; Juarros, a.; Retolaza, a.; Merino, S.; Ramírez, M. G.; Navarro-Fuster, V.; Villalvilla, J. M.; Boj, P. G.; Quintana, J. a.; Díaz-García, M. a. Highly Photostable Solid-State Organic Distributed Feedback Laser Fabricated via Thermal Nanoimprint Lithography. Microelectron. Eng. 2010, 87 (5-8), 1428-1430.

(253) Liu, X.; Klinkhammer, S.; Wang, Z.; Wienhold, T.; Vannahme, C.; Jakobs, P.; Bacher, A.; Muslija, A.; Mappes, T.; Lemmer, U. Pump Spot Size Dependent Lasing Threshold in Organic Semiconductor DFB Lasers Fabricated via Nanograting Transfer. Opt. Express 2013, 21 (23), 14362-14367.

(254) Shallcross, R. C.; Chawla, G. S.; Marikkar, F. S.; Tolbert, S.; Pyun, J.; Armstrong, N. R. Efficient CdSe Nanocrystal Diffraction Gratings Prepared by Microcontact Molding. ACS Nano 2009, 3 (11), 3629-3637.

(255) Hermann, S.; Shallcross, R. C.; Meerholz, K. Simple Fabrication of an Organic Laser by Microcontact Molding of a Distributed Feedback Grating. Adv. Mater. 2014, 26, 6019-6024.

(256) Ozin, G. a.; Hou, K.; Lotsch, B. V.; Cademartiri, L.; Puzzo, D. P.; Scotognella, F.; Ghadimi, A.; Thomson, J. Nanofabrication by Self-Assembly. Mater. Today 2009, 12 (5), 12-23.

(257) Maxim N. Shkunov; Z. Valy Vardeny; Matt C. DeLong; Randy C. Polson; Anvar A. Zakhidov; Ray H. Baughman; Shkunov, M. N.; Vardeny, Z. V.; DeLong, M. C.; Polson, R. C.; et al. Tunable, Gap-State Lasing in Switchable Directions for Opal Photonic Crystals. Adv. Funct. Mater. 2002, 12 (1), 21-26.

(258) Kim, S.-H.; Kim, S.-H.; Jeong, W. C.; Yang, S.-M. Low-Threshold Lasing in 3D Dye-Doped Photonic Crystals Derived from Colloidal Self-Assemblies. Chem. Mater. 2009, 21 (20), 4993-4999.

(259) Furumi, S.; Kanai, T.; Sawada, T. Widely Tunable Lasing in a Colloidal Crystal Gel Film Permanently Stabilized by an Ionic Liquid. Adv. Mater. 2011, 23, 3815-3820.

(260) Furumi, S.; Fudouzi, H.; Miyazaki, H. T.; Sakka, Y. Flexible Polymer Colloidal-Crystal Lasers with a Light-Emitting Planar Defect. Adv. Mater. 2007, 19 (16), 2067-2072.

(261) Brenner, P.; Fleig, L.-M.; Liu, X.; Welle, A.; Bräse, S.; Lemmer, U. Degradation Mechanisms of 
Polyfluorene-Based Organic Semiconductor Lasers under Ambient and Oxygen-Free Conditions. J. Polym. Sci. Part B Polym. Phys. 2015, 53 (15), 1029-1034.

(262) List, E. J. W.; Guentner, R.; Scanducci de Freitag, P.; Scherf, U. The Effect of Keto Defect Sites on the Emission Properties of Polyfluorene-Type Materials. Adv. Mater. 2002, 14, 374-378.

(263) Zhao, W.; Cao, T.; White, J. M. On the Origin of Green Emission in Polyfluorene Polymers: The Roles of Thermal Oxidation Degradation and Crosslinking. Adv. Funct. Mater. 2004, 14 (8), 783790.

(264) Costela, a.; García-Moreno, I.; del Agua, D.; García, O.; Sastre, R. Highly Photostable Solid-State Dye Lasers Based on Silicon-Modified Organic Matrices. J. Appl. Phys. 2007, 101 (7), 073110.

(265) Dunn, B.; Zink, J. I. Optical Properties of Sol-Gel Glasses Doped with Organic Molecules. J. Mater. Chem. 1991, 1 (6), 903-913.

(266) Yang, Y.; Turnbull, G. A.; Samuel, I. D. W. Hybrid Optoelectronics: A Polymer Laser Pumped by a Nitride Light-Emitting Diode. Appl. Phys. Lett. 2008, 92 (16), 163306.

(267) Foucher, C.; Guilhabert, B.; Kanibolotsky, a. L.; Skabara, P. J.; Laurand, N.; Dawson, M. D. HighlyPhotostable and Mechanically Flexible All-Organic Semiconductor Lasers. Opt. Mater. Express 2013, 3 (5), 584.

(268) Richardson, S.; Gaudin, O. P. M.; Turnbull, G. a.; Samuel, I. D. W. Improved Operational Lifetime of Semiconducting Polymer Lasers by Encapsulation. Appl. Phys. Lett. 2007, 91, 261104.

(269) Vannahme, C.; Klinkhammer, S.; Christiansen, M. B.; Kolew, A.; Kristensen, A.; Lemmer, U.; Mappes, T. All-Polymer Organic Semiconductor Laser Chips: Parallel Fabrication and Encapsulation. Opt. Express 2010, 18 (24), 24881-24887.

(270) Bornemann, R.; Lemmer, U. Continuous-Wave Solid-State Dye Laser. Opt. Lett. 2006, 31 (11), 1669-1671.

(271) Bornemann, R.; Thiel, E.; Bolívar, P. H. High-Power Solid-State CW Dye Laser. Opt. Express 2011, 19 (27), 26382-26393.

(272) Kéna-Cohen, S.; Wiener, A.; Sivan, Y.; Stavrinou, P. N.; Bradley, D. D. C.; Horsfield, A.; Maier, S. A. Plasmonic Sinks for the Selective Removal of Long-Lived States. ACS Nano 2011, 5, 9958-9965.

(273) Rabe, T.; Gerlach, K.; Riedl, T.; Johannes, H. H.; Kowalsky, W.; Niederhofer, J.; Gries, W.; Wang, J.; Weimann, T.; Hinze, P.; et al. Quasi-Continuous-Wave Operation of an Organic Thin-Film Distributed Feedback Laser. Appl. Phys. Lett. 2006, 89, 081115.

(274) Lehnhardt, M.; Riedl, T.; Scherf, U.; Rabe, T.; Kowalsky, W. Spectrally Separated Optical Gain and Triplet Absorption: Towards Continuous Wave Lasing in Organic Thin Film Lasers. Org. Electron. physics, Mater. Appl. 2011, 12 (8), 1346-1351.

(275) Schols, S.; Kadashchuk, A.; Heremans, P.; Helfer, A.; Scherf, U. Triplet Excitation Scavenging in Films of Conjugated Polymers. ChemPhysChem 2009, 10 (7), 1071-1076.

(276) Zhang, Y.; Forrest, S. R. Existence of Continuous-Wave Threshold for Organic Semiconductor Lasers. Phys. Rev. B - Condens. Matter Mater. Phys. 2011, 84, 241301(R).

(277) Giebink, N. C.; Forrest, S. R. Temporal Response of Optically Pumped Organic Semiconductor Lasers and Its Implication for Reaching Threshold under Electrical Excitation. Phys. Rev. B Condens. Matter Mater. Phys. 2009, 79 (7), 1-4.

(278) Sandanayaka, A. S. D.; Yoshida, K.; Inoue, M.; Qin, C.; Goushi, K.; Ribierre, J.-C.; Matsushima, T.; Adachi, C. Quasi-Continuous-Wave Organic Thin-Film Distributed Feedback Laser. Adv. Opt. Mater. 2016, n/a - n/a.

(279) Nakanotani, H.; Adachi, C.; Watanabe, S.; Katoh, R. Spectrally Narrow Emission from Organic Films under Continuous-Wave Excitation. Appl. Phys. Lett. 2007, 90, 231109.

(280) Mróz, M. M.; Sforazzini, G.; Zhong, Y.; Wong, K. S.; Anderson, H. L.; Lanzani, G.; CabanillasGonzalez, J. Amplified Spontaneous Emission in Conjugated Polyrotaxanes under Quasi-CW Pumping. Adv. Mater. 2013, 25 (31), 4347-4351.

(281) Reufer, M.; Riechel, S.; Lupton, J. M.; Feldmann, J.; Lemmer, U.; Schneider, D.; Benstem, T.; 
Dobbertin, T.; Kowalsky, W.; Gombert, a.; et al. Low-Threshold Polymeric Distributed Feedback Lasers with Metallic Contacts. Appl. Phys. Lett. 2004, 84 (17), 3262.

(282) Schols, S.; Van Willigenburg, L.; Steudel, S.; Genoe, J.; Heremans, P. Pulsed Excitation of OLEDs With a Remote Metallic Cathode. IEEE J. Quantum Electron. 2010, 46 (1), 62-67.

(283) Görrn, P.; Rabe, T.; Riedl, T.; Kowalsky, W. Loss Reduction in Fully Contacted Organic Laser Waveguides Using TE2 Modes. Appl. Phys. Lett. 2007, 91 (4), 041113.

(284) Gärtner, C.; Karnutsch, C.; Lemmer, U.; Pflumm, C. The Influence of Annihilation Processes on the Threshold Current Density of Organic Laser Diodes. J. Appl. Phys. 2007, 101 (2), 023107.

(285) Kuwae, H.; Nitta, A.; Yoshida, K.; Kasahara, T.; Matsushima, T.; Inoue, M.; Shoji, S.; Mizuno, J.; Adachi, C. Suppression of External Quantum Efficiency Roll-off of Nanopatterned Organic-Light Emitting Diodes at High Current Densities. J. Appl. Phys. 2015, 118, 155501.

(286) Hayashi, K.; Nakanotani, H.; Inoue, M.; Yoshida, K.; Mikhnenko, O.; Nguyen, T. Q.; Adachi, C. Suppression of Roll-off Characteristics of Organic Light-Emitting Diodes by Narrowing Current Injection/transport Area to 50 Nm. Appl. Phys. Lett. 2015, 106, 093301.

(287) Lehnhardt, M.; Riedl, T.; Weimann, T.; Kowalsky, W. Impact of Triplet Absorption and TripletSinglet Annihilation on the Dynamics of Optically Pumped Organic Solid-State Lasers. Phys. Rev. B - Condens. Matter Mater. Phys. 2010, 81, 165206.

(288) Kabra, D.; Lu, L. P.; Song, M. H.; Snaith, H. J.; Friend, R. H. Efficient Single-Layer Polymer LightEmitting Diodes. Adv. Mater. 2010, 22 (29), 3194-3198.

(289) Setoguchi, Y.; Adachi, C. Suppression of Roll-off Characteristics of Electroluminescence at High Current Densities in Organic Light Emitting Diodes by Introducing Reduced Carrier Injection Barriers. J. Appl. Phys. 2010, 108 (6).

(290) Rabe, T.; Görrn, P.; Lehnhardt, M.; Tilgner, M.; Riedl, T.; Kowalsky, W. Highly Sensitive Determination of the Polaron-Induced Optical Absorption of Organic Charge-Transport Materials. Phys. Rev. Lett. 2009, 102, 137401.

(291) Kena-Cohen, S.; Forrest, R. S.; Forrest, S. R. Room-Temperature Polariton Lasing in an Organic Single-Crystal Microcavity. Nat. Photonics 2010, 4, 371-375.

(292) Vannahme, C.; Christiansen, M. B.; Mappes, T.; Kristensen, A. Optofluidic Dye Laser in a Foil. Opt. Express 2010, 18 (9), 9280-9285.

(293) Liu, X.; Prinz, S.; Besser, H.; Wissmann, M.; Vannahme, C.; Guttmann, M.; Koos, C.; Lemmer, U. Organic Semiconductor Distributed Feedback Laser Pixels for Lab-on-a-Chip Applications Fabricated by Laser-Assisted Replication. Faraday Discuss. 2014, 174, 152-164.

(294) Mappes, T.; Vannahme, C.; Klinkhammer, S.; Bog, U.; Schelb, M.; Grossmann, T.; Hauser, M.; Kalt, H.; Lemmer, U. Integrated Photonic Lab-on-Chip Systems for Biomedical Applications. Proc. SPIE 2010, 7716, 77160R - 77160R - 10.

(295) Vannahme, C.; Klinkhammer, S.; Kolew, A.; Jakobs, P.-J.; Guttmann, M.; Dehm, S.; Lemmer, U.; Mappes, T. Integration of Organic Semiconductor Lasers and Single-Mode Passive Waveguides into a PMMA Substrate. Microelectron. Eng. 2010, 87 (5-8), 693-695.

(296) Bakal, A.; Vannahme, C.; Kristensen, A.; Levy, U. Tunable on Chip Optofluidic Laser. Appl. Phys. Lett. 2015, 107, 211105.

(297) Mappes, T.; Wienhold, T.; Bog, U.; Vannahme, C.; Christiansen, M. B. On-Chip Integrated Lasers for Biophotonic Applications. Proc. ISOT 2012, 12-13.

(298) Vannahme, C.; Klinkhammer, S.; Lemmer, U.; Mappes, T. Plastic Lab-on-a-Chip for Fluorescence Excitation with Integrated Organic Semiconductor Lasers. Opt. Express 2011, 19 (9), 8179-8186.

(299) Yang, Y.; Turnbull, G. a.; Samuel, I. D. W. Sensitive Explosive Vapor Detection with Polyfluorene Lasers. Adv. Funct. Mater. 2010, 20 (13), 2093-2097.

(300) Wang, Y.; Morawska, P. O.; Kanibolotsky, A. L.; Skabara, P. J.; Turnbull, G. A.; Samuel, I. D. W. LED Pumped Polymer Laser Sensor for Explosives. Laser Photon. Rev. 2013, 7 (6), L71-L76.

(301) Deng, C. M.; He, C.; Shi, L. Q.; Fu, Y. Y.; Zhu, D. F.; Cao, H. M.; He, Q. G.; Cheng, J. G. Turn-on Lasing 
Sensory Mechanism for Vapor Detection of Amines with Metallophenylporphrin Doped

Polyfluorene. 2011 16th Int. Solid-State Sensors, Actuators Microsystems Conf. TRANSDUCERS'11 2011, 124-127.

(302) Gaal, M.; Sax, S.; Plank, H.; Teuchtmann, M.; Rinnerbauer, V.; Hasenfuß, C.; Schmidt, H.; Hingerl, K.; List, E. J. W. Directly Imprinted Surface-Emitting Distributed Feedback Structure Polymer Sensor Laser Devices for Enhanced Oxygen Sensitivity. Jpn. J. Appl. Phys. 2008, 47 (1), 304-306.

(303) Lozenko, S.; Faye, D.; Zhang, H.; Lebental, M.; Lautru, J.; Zyss, J.; Lefevre, J.-P.; Leray, I. Geometrical Optimization of Organic Microlasers for Microfluidic Chemical Sensing. Appl. Phys. B 2014, 117 (1), 501-508.

(304) Smith, C. L. C.; Lind, J. U.; Nielsen, C. H.; Christiansen, M. B.; Buss, T.; Larsen, N. B.; Kristensen, A. Enhanced Transduction of Photonic Crystal Dye Lasers for Gas Sensing via Swelling Polymer Film. Opt. Lett. 2011, 36 (8), 1392-1394.

(305) Liu, X.; Stefanou, P.; Wang, B.; Woggon, T.; Mappes, T.; Lemmer, U. Organic Semiconductor Distributed Feedback ( DFB ) Laser as Excitation Source in Raman Spectroscopy. Opt. Express 2013, 21 (23), 28941-28947.

(306) Chen, Y.; Zhang, Y.; Laurand, N.; Kanibolotsky, A. L.; Gu, E.; Skabara, P. J.; Dawson, M. D. Organic Polymer Composite Random Laser Operating Underwater. Opt. Lett. 2012, 37 (24), 5160-5162.

(307) Liu, X.; Lebedkin, S.; Besser, H.; Pfleging, W.; Prinz, S.; Wissmann, X. M.; Schwab, P. M.; Nazarenko, I.; Guttmann, M.; Kappes, M. M.; et al. Tailored Surface-Enhanced Raman Nanopillar Arrays Fabricated by Laser- Assisted Replication for Biomolecular Semiconductor Lasers. ACS Nano 2015, 9 (1), 260-270.

(308) Morales-Vidal, M.; Boj, P. G.; Quintana, J. a.; Villalvilla, J. M.; Retolaza, A.; Merino, S.; Díaz-García, M. a. Distributed Feedback Lasers Based on Perylenediimide Dyes for Label-Free Refractive Index Sensing. Sensors Actuators B Chem. 2015, 220, 1368-1375.

(309) Haughey, a. M.; Guilhabert, B.; Kanibolotsky, a. L.; Skabara, P. J.; Burley, G. a.; Dawson, M. D.; Laurand, N. An Organic Semiconductor Laser Based on Star-Shaped Truxene-Core Oligomers for Refractive Index Sensing. Sensors Actuators, B Chem. 2013, 185, 132-139.

(310) Ramírez, M. G.; Villalvilla, J. M.; Quintana, J. a.; Boj, P. G.; Díaz-García, M. A. Distributed Feedback Lasers Based on Dichromated Poly(vinyl Alcohol) Reusable Surface-Relief Gratings. Opt. Mater. Express 2014, 4 (4), 733-738. 


\section{Acknowledgements}

MCG acknowledges financial support through the ERC Starting Grant ABLASE (640012) and the European Union Marie Curie Career Integration Grant (PCIG12-GA-2012-334407). AJCK acknowledges financial support by the German Federal Ministry for Education and Research through a NanoMatFutur research group (BMBF grant no. 13N13522).

\section{Biographies}

Alexander Kuehne studied chemistry at the University of Cologne and the University of Strathclyde, Glasgow. He received his PhD with a thesis on novel photo-polymers for optical applications from the University of Strathclyde before he conducted a postdoc stay in the group of Klaus Meerholz in Cologne. He then moved to the group of Dave Weitz at Harvard University to work on microfluidic and batch syntheses for semiconducting polymer colloids. He is currently a research group leader at the DWI Leibniz Institute for Interactive Materials in Aachen funded through a NanoMatFutur research group by the BMBF (Federal Ministry for Education and Research). In 2015 Alexander was elected as a member for the young section of the North-Rhine Westphalian Academy of Sciences and the Arts. He works on developing synthetic pathways towards new materials with designed properties in photonic-, electronicand bio-imaging applications.

Malte Gather studied physics and material sciences at RWTH Aachen University and Imperial College London. In 2008, he received his PhD from University of Cologne (Klaus Meerholz) with a thesis on crosslinkable organic semiconductors and organic LEDs. As a postdoc at University of Iceland (with Kristjan Leosson) and later as Bullock-Wellman Fellow at Harvard University (with SH Andy Yun) he worked on optical amplification in plasmonic waveguides and on biologically produced photonic devices, in particular on lasers formed by single live cells. He was assistant professor at Institut für Angewandte 
Photophysik of TU Dresden (Germany) from 2011 to 2013 before becoming a full professor at University of St Andrews (UK). He has received the Paterson Medal (Institute of Physics), the Rudolf-Kaiser Prize and the Daimler Benz Foundation fellowship and holds a Starting Grant from the European Research Council. His work on single cell lasers was recognized by the Institute of Physics as one of the top 10 breakthroughs in physics. Together with his interdisciplinary research team he explores nanophotonics and organic semiconductors, with a particular interest in applying them to biology. 